Historic, Archive Document

Do not assume content reflects current scientific knowledge, policies, or practices. 



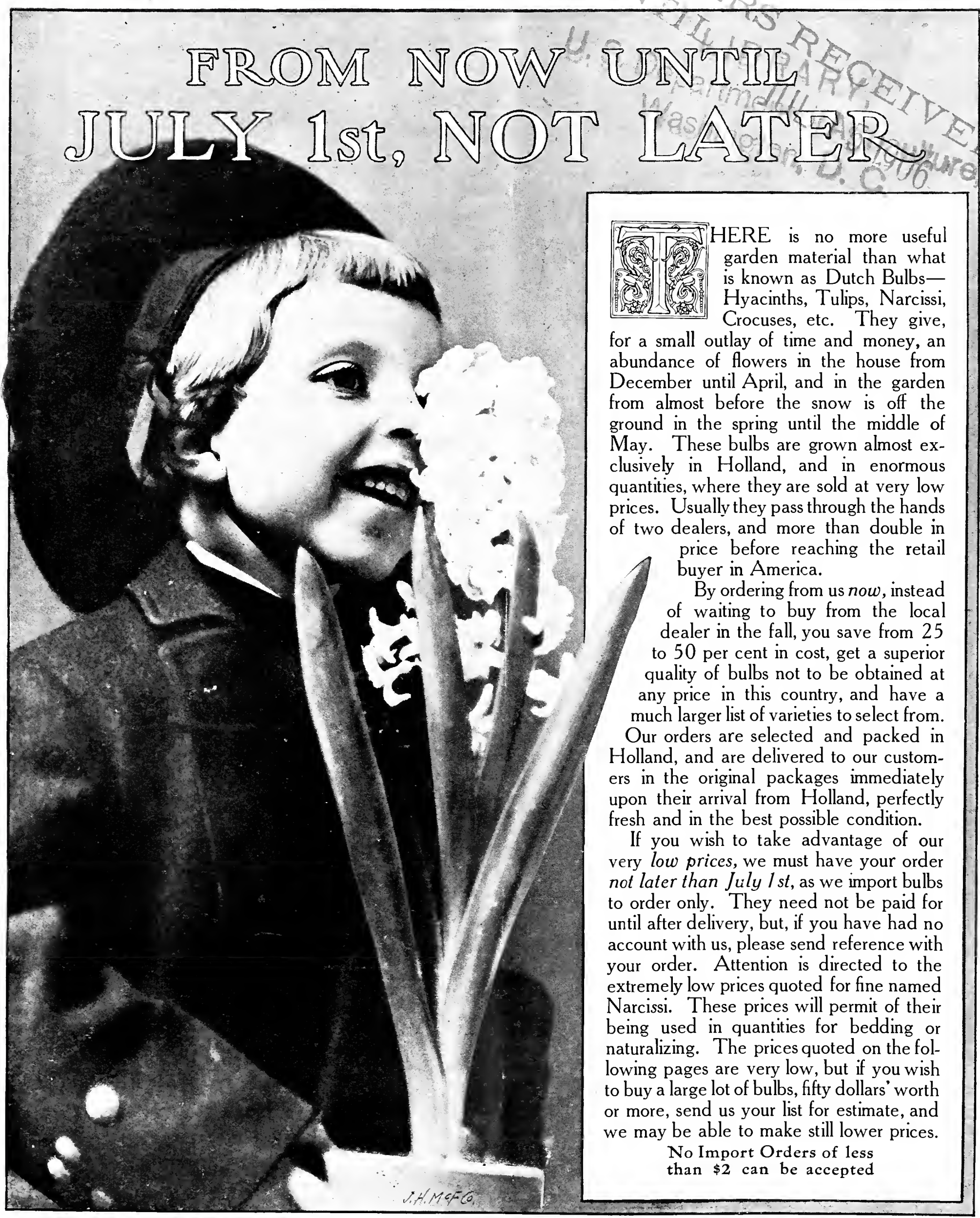

\section{ELLIOTT NURSERY COMPANY}

NURSERYMEN

HORTICULTURAL BUYERS' AGENTS
J. WILKINSON ELLIOTT, President

German National Bank Bldg., PITTSBURG, PA. 


\section{Terms of Payment, Etc. \\ PLEASE READ BEFORE ORDERING}

\section{Address all orders and letters to Springdale, Pa., but have money orders payable at Pittsburg}

HYACINTHS, TULIPS, CROCUSES, Ete.-The prices quoted in this list on all Duteh Bulbs, Bermuda Lilies and Roman Hyacinths are for advance orders, which must reach us not later than July 1, 1905 . To make these very low prices, which are, in many instances, lower than American Wholesale Prices, we must have our orders in advance, as we cannot take any risk of having stock remain unsold on our hands. The bulbs will be delivered at the proper season, and need not be paid for until after delivery. Prices quoted include the 25 per cent duty and all charges except freight or expressage from Pittsburg.

TIME OF DELIVERY.-Bermuda Lilies arrive in August; Roman Hyacinths, Freesias, Candidum Lilies, the latter part of August or early in September; Dutch Bulbs, Hyacinths, Tulips, etc., about October 1 to 10 . In ordering, please state whether you wish bulbs shipped as they arrive, or all in one shipment, about October 1 to 10 . Dutch Bulbs may be sent safely by freight, but we advise shipping bulbs or plants that arrive in November by express. In absence of instructions we will send all bulbs in one shipment, except Japanese Lilies and Lily-of-the-Valley, which will be shipped upon arrival, in October and November. Some dealers make a point of offering Dutch Bulbs for sale very early in the fall, which often necessitates the shipping of bulbs from Holland before they are properly ripened-a frequent cause of failure to bloom. We do not usually try to deliver Dutch Bulbs before October 1, which is quite early enough.

SHIPPING INSTRUCTIONS.-Please send full shipping instructions. State whether goods are to be shipped by freight or express, and by what route. In the absence of instructions, we will ship by whatever method and route we think best.

TERMS OF PAYMENT.-Our terms of payment are invariably eash on delivery, which does not mean that we ship C. O. D., as we will not do so under any circumstances. We deliver the goods and mail bills at the same time, and expect payment within a few days. People unknown to us will please send references with their orders. Those who wish to send cash in advance with their orders will be allowed a discount of five per cent from the prices quoted on this list on all orders received before July 1, 1905.

GUARANTEES. - We guarantee all trees, shrubs, plants and bulbs furnished by us to be first-class, true to name and delivered in good condition when shipped by express. Stock is shipped by freight at purchaser's risk, and eustomers must make claim on railroad companies for any loss caused by delay.

We do not guarantee stock to grow, or results in any way. No complaint will be entertained that is not made immediately upon receipt of stock.

There are so many causes for failure over which we have no control that we can assume no responsibility after stock is delivered in good order. Poor soil, unfavorable weather, ignorant or careless culture, all contribute to failure, and all are beyond our control.

EARLY ORDERS. - The earlier orders are given, the better we can have them filled. Some of our customers are now giving us orders to be delivered next spring. Our representative usually sails for Europe early in July, and personally selects the stock for all import orders received by July 1.

CLUB ORDERS. - No smaller quantities will be supplied than those quoted in the list, except that six bulbs will be furnished at dozen price, fifty at hundred price and five hundred at thousand price; but it is suggested, where these quantities ale too large, that two or three friends or neighbors could combine their orders with advantage, and save transportation charges.

PLEASE NOTICE the date of delivery and the limit of time for receiving orders for the different classes of stock. Often orders are sent us too late in the season to be filled.

EXPERIMENTAL GROUNDS.-For our own information and for the benefit of our customers, we have established experimental grounds where all promising novelties are tested. We also grow all varieties of trees, shrubs and hardy plants generally in the trade, so that we can have full information as to their respective merits.

UNITED STATES AND AMERICAN EXPRESS SHIPMENTS.-Our Pittsburg shipping is all done from Cheswick, a local station which is an exclusive Adams Express office. We can not ship direct by any other company, but we can ship by Adams Express, care of American, United States, Wells Fargo, or Pacific Company, from Pittsburg.

A discount of 20 per cent is allowed from regular express rates on plants and bulbs, and customers should see that this discount is allowed when paying charges. 


\section{A GARDEN OF LILIES}

A correspondent complains that we have told him too often of the lawyer's garden; but it is still the best garden in this vicinity, and a good garden cannot be told about too often.

Our friend, the lawyer, has a garden of Lilies. Many other things he has in his garden-great banks of Mollis and Ghent azaleas that are worth a long journey to see; an entire hillside is covered with hybrid rhododendrons and Kalmia latifolia; tulips, daffodils and crocuses are everywhere in the spring, and the glorious show of Japanese irises in June is a sight not easily to be forgotten - but he has Lilies by the hundred and by the thousand, and in so many varieties that from June until November there is always a fine display of Lily flowers. Sueh a garden! By daylight it is splen did, by moonlight it is fairyland, and the air is filled with fragrance. Such a garden to visit, as we do visit it and travel forty miles a dozen times a year, and come away with our arms filled with great stalks of Lily bloom. For this is a garden in which there are always flowers to eut and to spare. It is not the miserable garden of bedding plants in which its owner finds it difficult to eut a little nosegay to give to a friend. We wish you who are content to grow such commonplace things of so little beauty as cannas, geraniums, coleus and alternantheras could visit this garden of Lilies, and then say what excuse you have to offer for your poor taste. Not that of cost, for Lily bulbs are to be had for as little money as bedding plants, and as Lilies are hardy, their first cost is their only cost, while bedding plants must be bought every season.

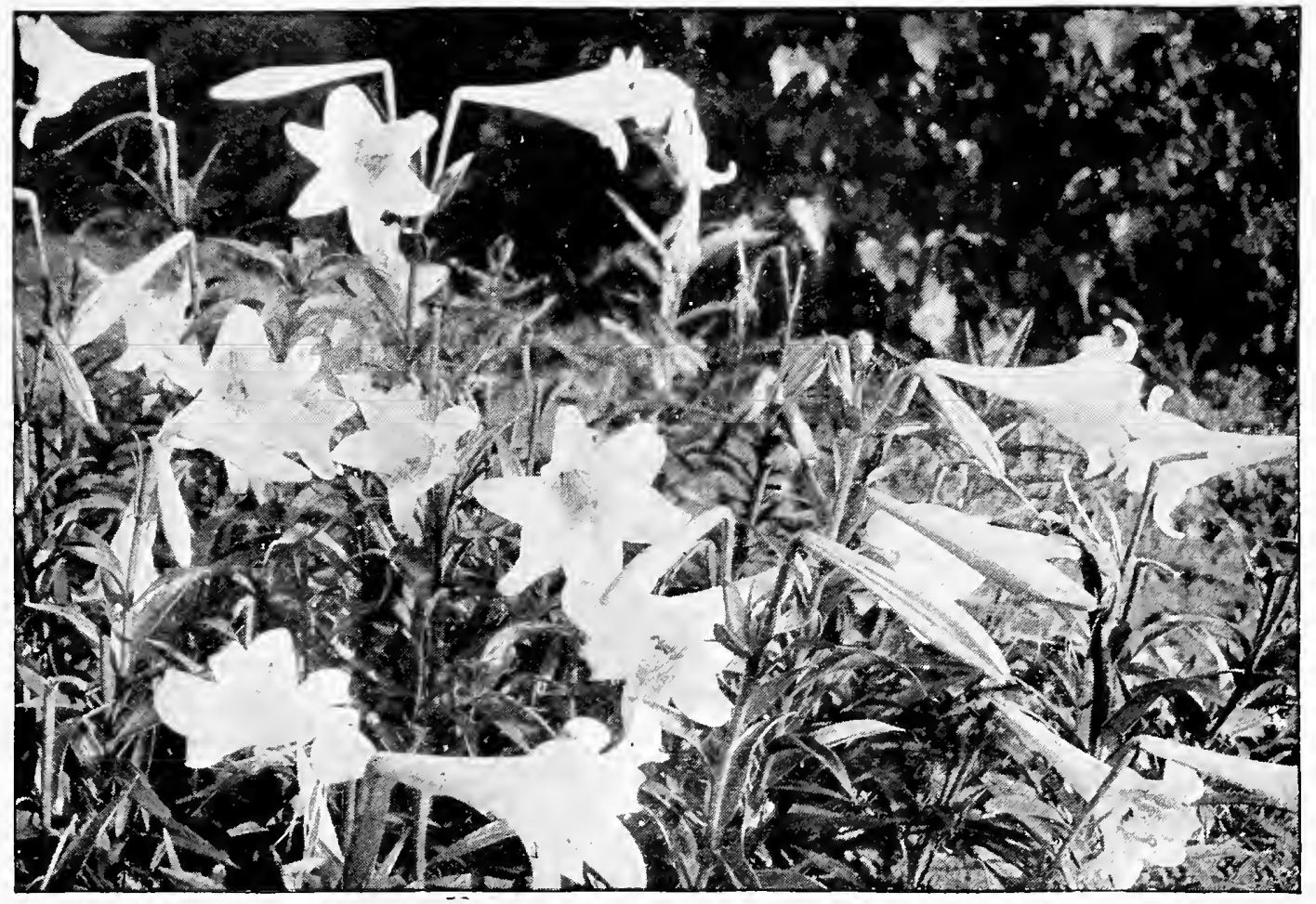

LILIUM LONGIFLOLUM

\section{Fall Planting of Lilies}

NOTICE.-To secure Fall dellvery of Llly bulbs, we should recelve orders not later than July I

The great cause of failure and disappointment in planting Lilies is that they are kept out of the ground too long. The Auratum, Rubrum and Album, the most effective and desirable of all Lilies, are imported from Japan in the fall, and usually do not arrive in this country until December, and, of course, cannot be planted outdoors until the following spring. This long exposure greatly weakens the bulbs, and the flowers are not satisfactory. Never buy Lilies that are offered for sale in seed or floral stores; they are so weakened by exposure that they are worthless. This fall we are importing an enormous quantity of Lilies from Japan, and instead of having them come by the slow, but inexpensive, all-water route, they are shipped by fast steamer to Canada, and from thence by special tea train, which usually enables us to have the bulbs in Pittsburg in October. Immediately upon their arrival they will be delivered to our customers in ample time for planting this fall.

Formerly we guaranteed October delivery of Japanese Lilies, and usually succeeded in delivering them in October. This year we have made contracts requiring October delivery, with penalties for failure; but while we hope to deliver all Japanese Lilies by the end of October or early in November, in ample time for fall planting, we will not guarantee it. In the event of bulbs not arriving in time for fall planting, we will have them repacked in sand and stored in a cold cellar until spring, and deliver them as soon as the weather will permit. Lilies handled in this way will give just as good results as if planted in the fall.

Whenever delivered, we guarantee Lily bulbs, excepting rare varieties of Auratums, to be sound, in first-class condition, true to name and strictly first-class in every respect.

We have gone to considerable trouble to give our customers the opportunity of planting Japanese Lilies under the most favorable conditions, for we know of no flowers that are more strikingly beautiful and effective, or that will give more pleasure and satisfaction to the planter. We are not only supplying bulbs of very superior quality, but we are also selling them for onehalf regular retail prices, which you can confirm by referring to any seedsman's or plantsman's catalogue you may have at hand.

Lilies like a light, rich, well-drained but moist soil, and a partially shaded location. If planted in full exposure to the sun, the bed should be carefully mulched with 2 or 3 inches of rotted manure, leaves, sphagnum or moss. They thrive and are very effective when planted thickly among rhododendrons or azaleas, and may be used to advantage in any open space that may be in the shrubbery. Bulbs should be planted 6 inches deep and care taken that no manure comes in direct contact with them. Planted in quantity, they may be had in bloom from June until frost, and bloom freely the first season after planting.

Rubrum, Melpomene, Album and many other Lilies will thrive and increase in almost any soil, but unless the.conditions are very favorable, Auratum Lilies deteriorate and a percentage will be lost every year; but they are so truly splendid, and are so inexpensive at the low prices at which we offer them, that they will be found to be the best garden investment that can be made. Try these Lilies, and send your order at once, so that they can be sent to you immediately upon their arrival from Japan. Longiflorums are similar in appearance to the Bermuda Easter Lilies, but are much more satisfactory for outdoor planting, as they are perfectly hardy. 


\section{PRICES OF LILIES}

These prices include all charges except freight or express charges from Pittsburg. The measurements given refer to circumference of bulbs, but the Japanese eridently use an elastic tape, as the bulbs never quite come up to the measurements given. I give these sizes as they are generally used by the trade.

Perdoz. $\quad 100 \quad 1,000 \quad$ Speciosum album. White. 8 to 9 ir

9 to 11 inches.......................... $150 \quad 1100$

11 to 13 inches.......................... 2 2 $75 \quad 2000$

11 to 13 inches, selected bulbs.............. $3325 \quad 2200$

Longiflorum. Has large, pure white, trumpet-

shaped flowers like the Bermuda Easter

Lily, but is perfeetly hardy.

5 to 7 inches........................ 55

6 to 8 inches............................... 75

7 to 9 inches............................. 100

9 to 10 inches.......................... 1 60

$\begin{array}{rrrr}3 & 50 & \$ 25 & 00 \\ 4 & 50 & 33 & 00 \\ 7 & 00 & 52 & 00 \\ 12 & 00 & 85 & 00\end{array}$
9 to 11 inches................................ $250 \quad 1800$ 11 to 12 inches.................................. $350 \quad 2500$ Speciosum Melpomene. Similar to Roseum or Rubrum; more brilliant in color.

7 to 9 inches.............................. 135900

9 to 10 inches.................................. 1 $75 \quad 1300$

Speciosum rubrum, or roseum. Pink.

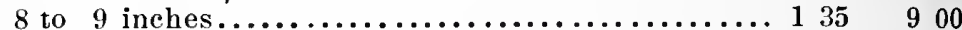

9 to 11 inches...............................................

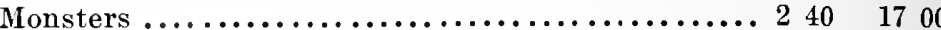

\section{RARE VARIETIES OF AURATUM LILIES}

The following varieties of Auratum Lilies are exquisitely beautiful, but difficult to import in good condition. They will be imported at the risk of customers only, and delivered immediately on arrival. I first saw these Lilies some years ago in London, when they were selling for a guinea $(\$ 5)$ a bulb.

Auratum macranthum. A grand variety of re- $\begin{array}{lll}\text { Each Doz. } & 100\end{array}$ markable strength and vigor; immense, pure ivory - white flowers, with a golden band

throngh each petal. Large bulbs, 8 to 10 inches................................. \$0 $50 \quad \$ 500 \quad \$ 36 \quad 00$

Auratum pictum. A very choice variety: pure white, with red and yellow band through each

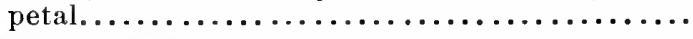

Auratum platyphyllum. A remarkably. large Lily of great vigor.............................

$35 \quad 350 \quad 2800$

$50 \quad 500$
Auratum virginale album. Exquisitely beautiful; large flowers of the purest white, with a narrow band of yellow th rough center of each petal.\$0 $\quad \begin{array}{lllll}60 & \$ 6 & 00 & \$ 15 & 00\end{array}$ Auratum vittatum rubrum. Magnificent flowers, 10 to 12 inches across; clear waxy white, with broad crimson stripe through the center of

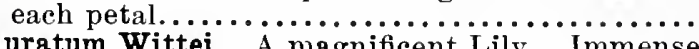
flowers of the purest white, with a wide yellow stripe through the center of each petal; very tall-growing and free-blooming...............
Each Doz. 100

$\begin{array}{lll}75 & 750\end{array}$

$75 \quad 750$

\section{OTHER JAPANESE LILIES}

Varieties marked with an asterisk $\left(^{*}\right)$ can not be delivered until late in the fall or early in the spring.

*Alexandrae. A Japanese Lily with flowers similair to Each Doz. Longiflorum, but very much larger and finer in every respect; very sweet-scented.

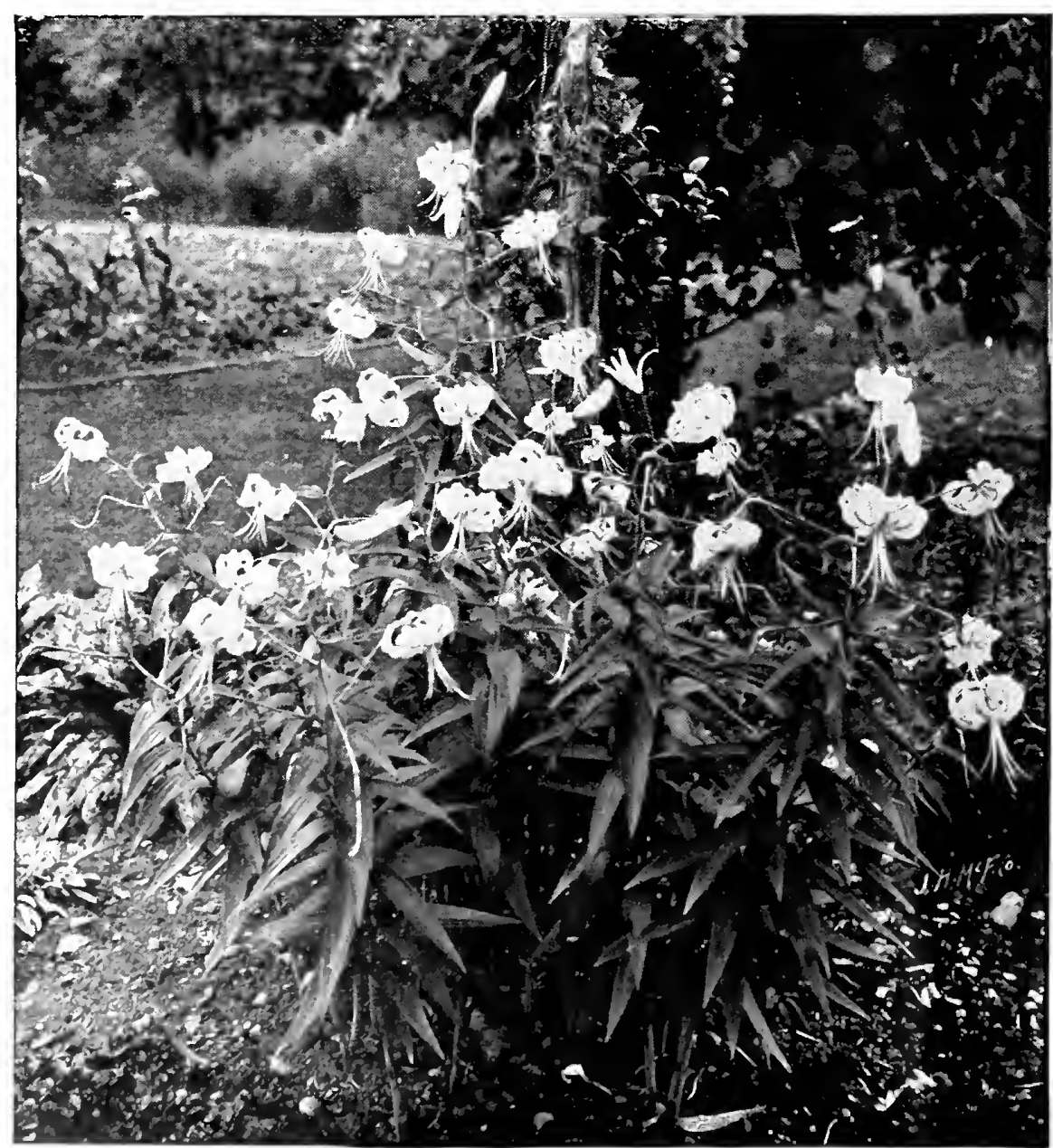

LILIUM SPECIOSUM ALBUM

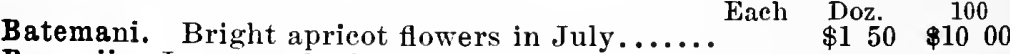
Brownii. Japanese bulbs. An extremely handsome lardy Lily, with large trumpet-shaped flowers like those of Longiflorum or Bermuda Easter Lily; pure white inside, but the outer part of the petal is a beautiful purplish brown.\$0 $35 \quad 3 \quad 50 \quad 3000$ Iansoni. A handsome variety flower. ing in June. Has bright rich yellow flowers; one of the best Lilies in cultivation. Bulbs sometimes re. main dormant after planting,but will come up vigorously the next season.

* Henryi. A new Japanese Lily that has made a sensation in Europe. It has the same form and general appearance of the Speciosum varieties, but the flowers are bright orange yellow. Scarce, and delivery is doubtful...

Krameri. Distinet from all other Lilies. Large flowers of a soft, beautiful rose color................

Leichtlini, Red. Orange - red, with crimson spots....................

Leichtlini, Yellow. Neat and elegant habit; flowers pure canary-yellow,

with erimson spots...............
Rubellum. This is a beautiful new Lily similar to Krameri. It is unknown in this country, but in England, where it has been fully tested, it is highly praised, and it seems likely to become as popular as the Speciosum varieties...............

$65 \quad 700 \quad 5000$

$\begin{array}{lllll}75 & 8 & 00 & 55 & 00\end{array}$

$135 \quad 1000$

$\begin{array}{llll}40 \quad 4 & 00 \quad 3000\end{array}$

$\begin{array}{lllll}25 & 2 & 75 & 20 & 00\end{array}$

\section{EUROPEAN-GROWN} October dellvery. Orders recelved until August I

Brownii. Dutch bulbs. An extremely handDoz. $\quad 100$

some hardy Lily, with large trumpet-shaped flowers like those of Longiflorum or the Bermuda Easter Lily; pure white inside, but outer part of the petals is beautiful purplish brown. This is a very choice Lily, but good bulbs of it are scarce....each, 60c...\$6 50 


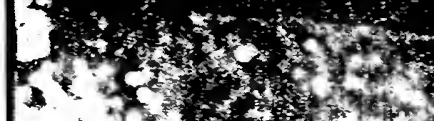

A

m.

to

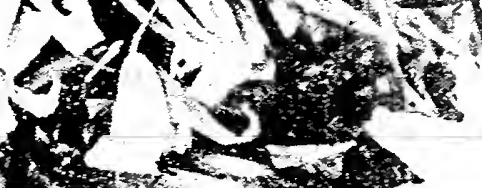

4 , th

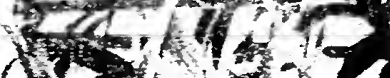

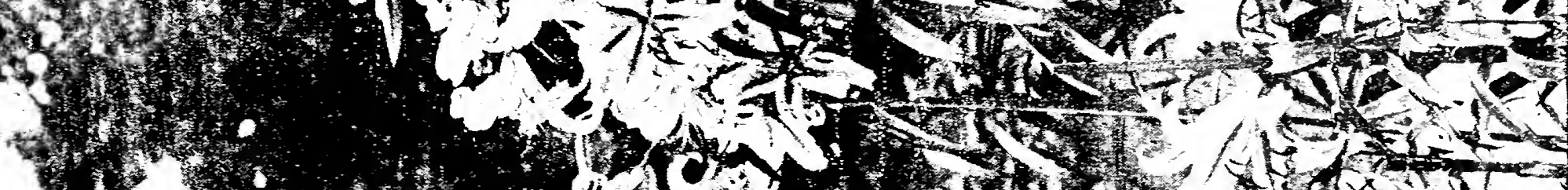

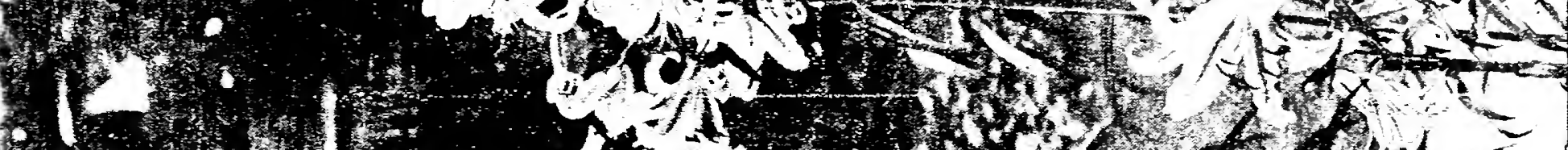

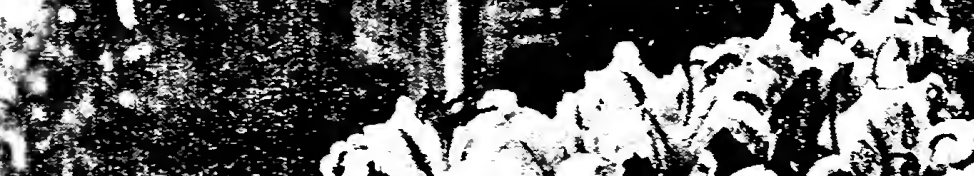

集零,

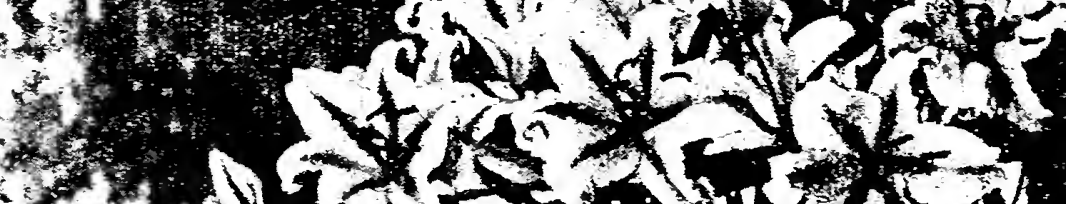

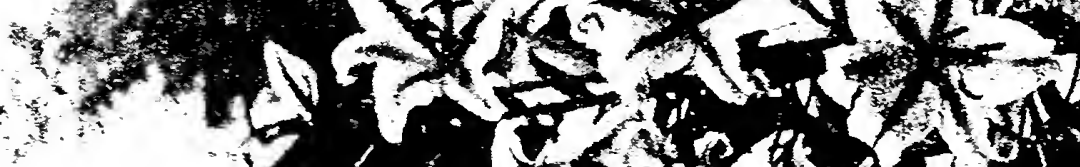

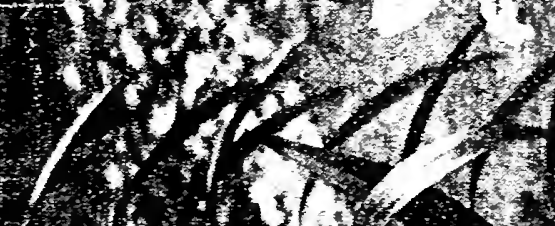

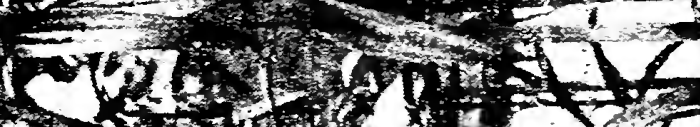
4040 os

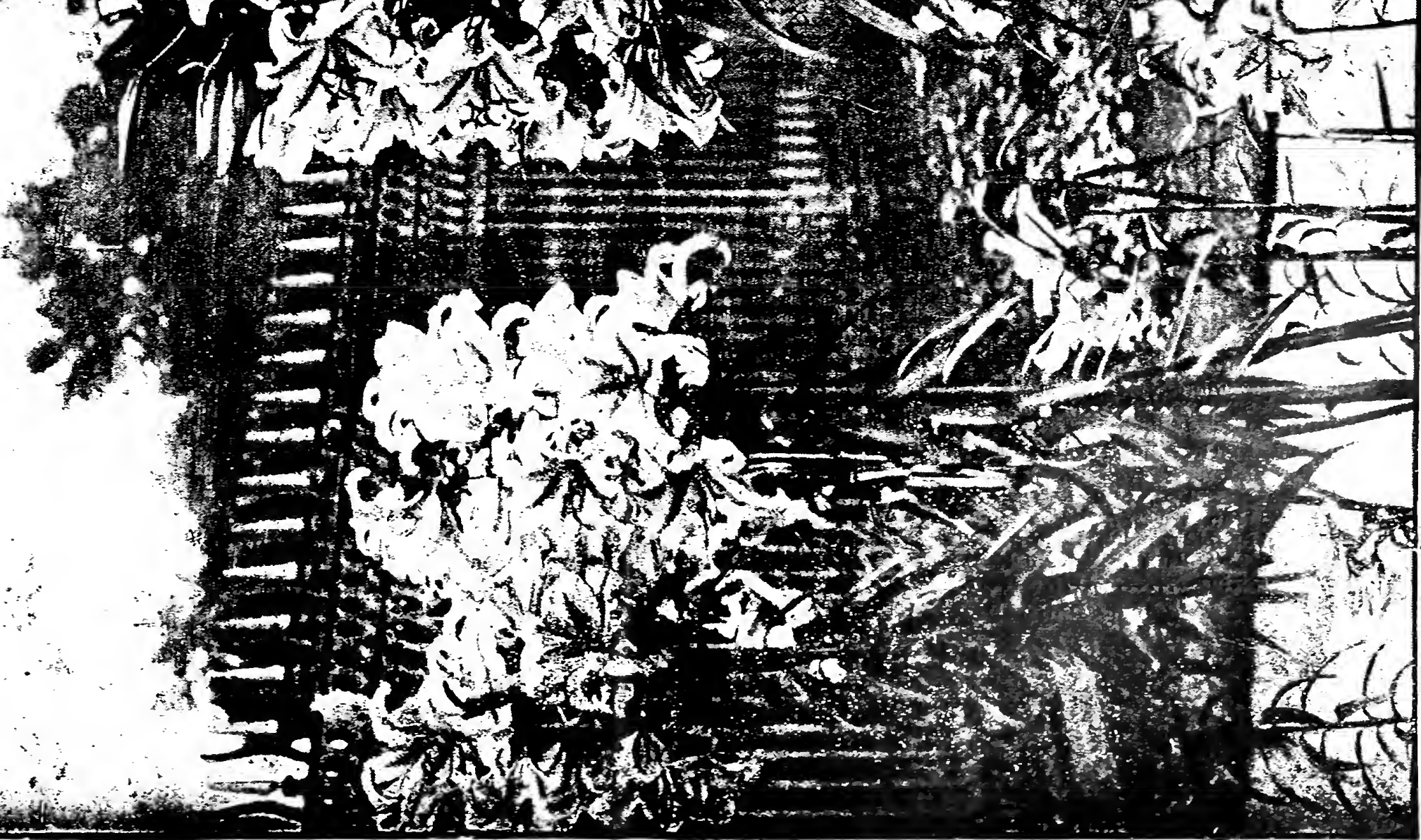


Bulbiferum. True. Deep orange-red.............. Doz. $\begin{array}{ccc} & 100 \\ 75 & \$ 19 & 00\end{array}$ Candidum (Madonna or Annunciation Lily). This is the old-fashioned garden Lily, and one of the most beautiful. It should nerer be plaated later than September 15 , as it makes a fall growth............... 150 chalcedonicum. The Scarlet Turk's Cap Lily............. each, 55 ets...5 55 Colchicum. Rich citron-yellow, spotted with black.... eacin, 65 cts... 650

Croceum. Orange-red, spotted black; distinct........ Doz. $\$ 200 \quad \$ 1300$

Excelsum. Another choice but scarce Lily. The stately form, beauty of color, and delightful fragrance of this variety has made it a favorite wherever grown. It grows 4 to 6 feet high, and produces six to twelve flowers of delicate light buff color, blooming in June and July.......................each, 75 ets... 850 Giganteum. A remarkable Lily, growing 12 to 14 feet high ; it requires an expert gardener to bloom it. Large bulbs.................each, $\$ 1.50 .1500$ Longiflorum Takesima. Pure white trumpet and having blackish stems.............. 160 Martagon (Turk's Cap Lily). Purple...... 160

Martagon album. Pure white...each, \$2.25..24 00 Martagon dalmaticum. Blackish purple.....

Pardalinum. Golden orange, spotted with maroon-purple......................... 150

Parryi. Large, pale yellow flowers..each, $\$ 1 . .1000$ Speciosum Schrymakersii. Beautiful rosy white, suffused and spotted with rosy crimson .......................... 200

Thunbergianum (elegans) aurantiacum. Dark orange-brown. The Thunbergianums are all of the easiest eulture, and bloom in June ........................... 125

Thunbergianum aureum. Yellow; dwarf.... 65

Thunbergianum citrinum. Citron-yellow .... each, 60 cts...6 600

Thunbergianum fulgens. Red, shaded with orange; one of the best................. 3 j0

Thunbergianum Van Houttei. Very large, scarlet-purple; very fine................ 150

Tigrinum flore pleno. The Double Tiger Lily. The only double Tiger Lily worth growing.

Tigrinum simplex. The well-known Single Tiger Lily. Of the easiest culture and worthy of general planting on account of stately habit and fine effect in the landscape...............................

Tigrinum splendens. Improved Single Tiger Lily................................

Umbellatum erectum. Scarlet, with brown spots............................ 150 Umbellatum grandiflorum. Orange, with brown spots....................... 150

Umbellatum incomparable. Dark red, with brown spots.......................... 180

Umbellatum, Fine Mixture............... 125

Wallichianum. A magnificent Lily, with immense white trumpet flowers, suffused with yellow; very scarce.......... each, $\$ 2.50 .$.

\section{NATIVE AND AMERICAN-GROWN LILIES}

(October Delivery)

\begin{tabular}{|c|c|c|}
\hline $\begin{array}{l}\text { Burbank Hybrids, Mixed .............. each, } \$ 1.25 \ldots \\
\text { Canadense flavum. Our dainty, beautiful native Lily. }\end{array}$ & $\begin{array}{l}\text { Doz. } \\
1200\end{array}$ & 100 \\
\hline Gracetul and charming yellow flow & 100 & $\$ 700$ \\
\hline $\begin{array}{l}\text { Canadense rubrum. Red flowers.... } \\
\text { Canadense, Mixed. }\end{array}$ & $\begin{array}{rr}100 \\
75\end{array}$ & \\
\hline $\begin{array}{l}\text { Columbianum, One of the most elegant and graceful } \\
\text { of Lilies. Orange-red flowers, spotted with crimson. }\end{array}$ & 250 & 1600 \\
\hline Elegans bicolor, Apricot, spotted............... & 175 & 1100 \\
\hline $\begin{array}{l}\text { Elegans robusta. Crimson-black; fine............... } \\
\text { Grayi. A small native Lily; very dainty and rare; the }\end{array}$ & 175 & 1100 \\
\hline 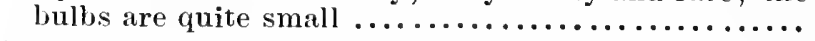 & 300 & 2000 \\
\hline Humboldtii. Orange-red, with claret-colored dots..... & 250 & 1600 \\
\hline $\begin{array}{l}\text { Philadelphicum. Native Lily. Orange-red, black spots. } \\
\text { Philippensis. This rare and beautiful Lily is a native } \\
\text { of one of the Philippine Islands. The flowers resem- } \\
\text { ble the Longiflorum, but are larger and very nuch } \\
\text { longer. The plant is extremely graceful and very }\end{array}$ & 100 & 700 \\
\hline 20 & & \\
\hline
\end{tabular}

Burbank Hybrids, Mixed ............each, $\$ 1.25 \ldots \$ 1200$ Canadense flavum, Our dainty, beautiful native Lily.

Graceful and charming yellow flowers ............................

Canadense rubrum. Red flowers................ 100

Columbianum. One of the most elegant and gracefu

of Lilies. Orange-red flowers, spotted with crimson.

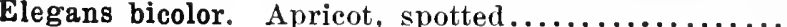

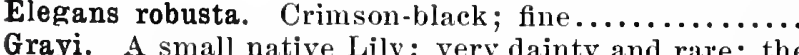

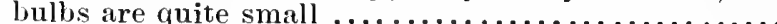

Humboldtii. Orange-red, with claret-colored dots.....

Philadelphicum. Native Lily. Orange-red, black spots. of one of the Philippine Islands. The flowers resem ble the Longiflorum, but are larger and very mucl free-flowering ..................each, 30 cts...
Superbum. In a collection of best plants of all countries, our native Superbum Lily would deserve a first place. In deep, rich soil it often grows 8 feet high, with twenty to thirty flowers. It is of the easiest culture, and may be grown as a wild flower in any swampy or rough part of a place where the grass is not mown. We have seen it growing by the thousand in swamps and meadows, but it well repays the highest garden culture. Extra selected bulbs .......... $\$ 1 \quad 50 \quad \$ 800$ Second size ........................... 1000

Tenuifolium. A beautiful, graceful Lily, with crimson reflex flowers. One of the choicest Lilies, but dies out in a year or two, but can be renewed from seed. Blooms in June..............................

Wallacei. Very free-flowering, hardy and showy; each bulb sends up many stems, which bear sereral lovely vermilion-orange flowers ......................

Washingtonianum. Very fragrant flowers, changing from pure white to various shades of purple or lilac. 25018 (1)

\section{ELLIOTT NURSERY COMPANY}

J. HORACE MCFarLand COMPan Hoaticultural Painteas
Hapaisquac. PEnNGyLania

\section{J. WILKINSON ELLIOTT, President}

GERMAN NATIONAL BANK BUILDING
PITTSBURG, PA. 


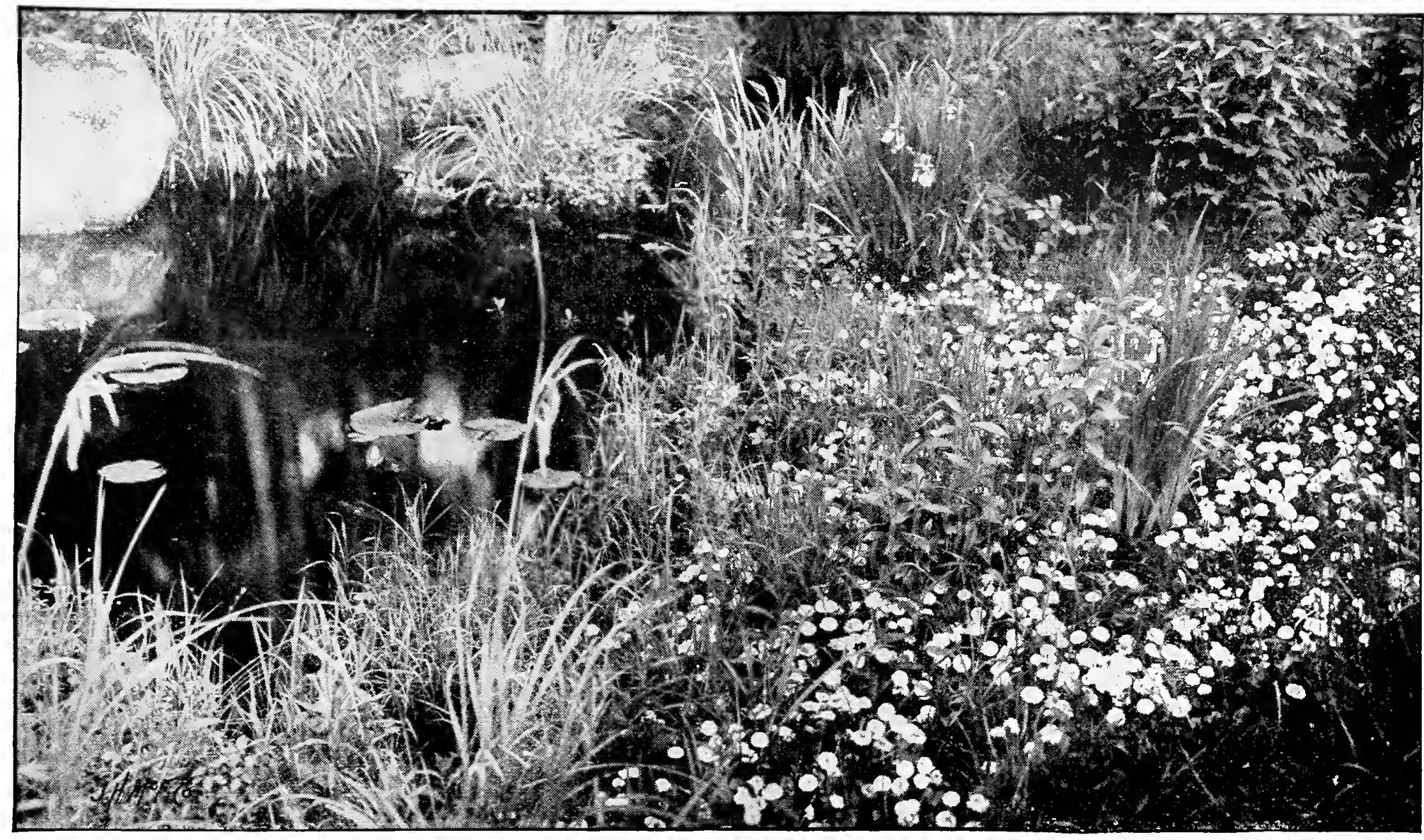

BELlis PERENNIS NATURALIZED ON THE BANK OF A POND

\section{"Flowers by the Ten Thousand}

THE EASIEST, THE LEAST EXPENSIVE AND THE MOST ARTISTIC WAY OF GROWING MYRIADS OF FLOWERS

\section{By J. WILKINSON ELLIOTT}

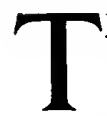

HE most inspiring floricultural idea of the last quarter of a century is the naturalizing of flowers by the thousand in situations where they need absolutely no care after planting. It is the easiest kind of gardening, for there is no weeding, watering, hoeing, staking or tying. It is the most artistic form of gardening, because the flowers fit perfectly into the landscape. It is the most effective kind of gardening, becanse nothing can surpass in beauty a continuous sheet of flowers all of the same variety. (No matter how numerous they may be, these wildings never seem gaudy or vulgar.) And, finally, it is the least expensive way of getting hosts of flowers-flowers like the stars of the Milky Way in multitude. A thousand narcissus bulbs! The thought takes one's breath away; yet a thousand bulbs of the poet's narcissus cost only five dollars, -a mere nothing compared with the vision of loveliness which it makes possible. And the first cost is the only cost. Compare the frontispiece of this magazine with the pretentious "Italian" gardens of today, which cost a fortune to maintain and never fit into an American landscape. Which style do you prefer? The accompanying illustrations must be a revelation, even to the initiated, of the wonderful pictures that have already been created in America, by the wholesale naturalization of flowers in woods and meadows.

Small city places do not offer many opportunities for naturalizing, but some of the spring flowers ean be used in this way on the smallest lots. Snowdrops and Scilla Sibivica can be planted in the grass of the most elosely shaven lawn; they are so dwarf and bloom so early that the bulbs ripen perfectly and will continue to bloom year after year. This is not true of erocuses, which are frequently planted on lawns. If the grass is mown, the crocuses must be replanted at least every two years.

When small bulbs are planted on lawns, care must be taken to arrange them in natural-looking groups. Often I see crocuses scattered over the entire surface of a lawn a foot or two apart; the effect is extremely bad. In naturalizing bulbs or hardy plants, each variety should be held together in irregular-shaped groups, which should be elosely planted in the eenter and more thinly as the margin is approached (see bluet picture). It is a good plan to scatter the bulbs over the surface of the ground before planting any of them. I stand in the center of the proposed group, dropping some of the bulbs at my feet and throwing others out in every direction, planting them where they fall. Circular groups should be avoided. They may be made of almost any irregular shape but always longer than they are broad. The arrangement largely depends upon the situation; a bay or recess in the shrubbery may be thiekly and entirely filled with one variety of bulbs, a sloping bank may be a mass of narcissi or tulips, or an orchard in which the grass is not mown until after July first will afford opportunities for many groups and a succession of bloom for two months or more.

The great advantage of using flowering bulbs in this way is that the plantings are permanent and need never be renewed but increase in size and beauty year after year, which is much more satisfactory than the present annual waste of millions of bulbs used for inartistic beds on the lawn. These bulbs are all destroyed, as they must be removed before they are ripened, in order to plant the undesirable bedding plants which follow them. It must always be remembered that the foliage of bulbs must not be cut off before it has ripened, but this does not prevent them from being planted in orehards and meadows, as the foliage is ripened by the time the grass is ready to cut for hay.

The only tool we use in planting bulbs is a good, strong garden trowel, with-which a hole is dug for each bulb. We cover *From Country Life in America. Copyrighted, 1904, by Doubleday, Page \& Co. 


\section{Flowers for the House from Christmas until Easter}

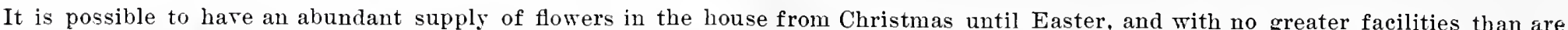

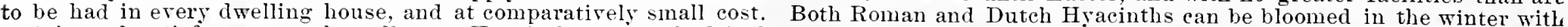

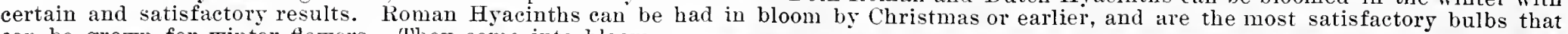
can be grown for winter flowers. They come into bloom quickly, and it is almost impossible to fail with them. 'The most delightful way to use them is to plant a quantity in a fern dish, jardinière or other receptable suitable for using for a centerpiece for the dining table. Fern dishes are made in a rariety of styles, and cost from $\$ 2$ or $\$ 3$ up to $\$ 25$ or $\$ 30$ for heary silver plated ones such as are made by the Gorham Manufacturing Company. The bulbs are not planted directly in the fern dish, but in a zinc pan, whic $h$ is made to fit inside of the fern aish. Extra zinc yans can be had, and when Roman Hyacinths are to be grown, from six to twelre extra pans should be used. The Roman Hyacinth should be pianted in the pans in the fall, from September 1 until the last of Norember, at intervals of two weeks.

The soil used should be light and rich, one-third good soil, one-third saud, and one-third thorougbly rotted manure, and the bulbs planted thickly, so that they almost touch. As soon as planted, they should be well watered and then set away in the coolest part of the cellar, and not near the furnace or any fire heat. If the cellar is a light one, the bulbs should be covered with boxes, canras, or anything that is convenient to keep them dark. If the soil gets dry it can be watered, but not oftener than once in two weeks. Roman Hracinths may be brought into the light and heat about December 1, and will be in bloom in about two weeks. After bringing into the liring-room they should be freely watered. By bringing a pan or more of bulbs from the cellar every ten days, a succession of flowers for the table can be had all winter and spring. A centerpiece of growing Roman or Dutch Hyacinths makes the most beautiful table decoration that can be obtained, does not cost one-fifth as much as cut-flowers, and will last in perfect beauty for ten days or more, while cut-flowers are often faded before a dinner is over. The pans for growing Roman Hyacinths can be from 3 to 5 inches deep, but pans for Dutch Hyacinths should not be less than 4 inches deep. Do not attempt to force cheap mixed Hyacinths; the results will not be satisfactory.

Roman Hyacinths have small single white, pink or blue flowers. They bloom profusely, having from four to

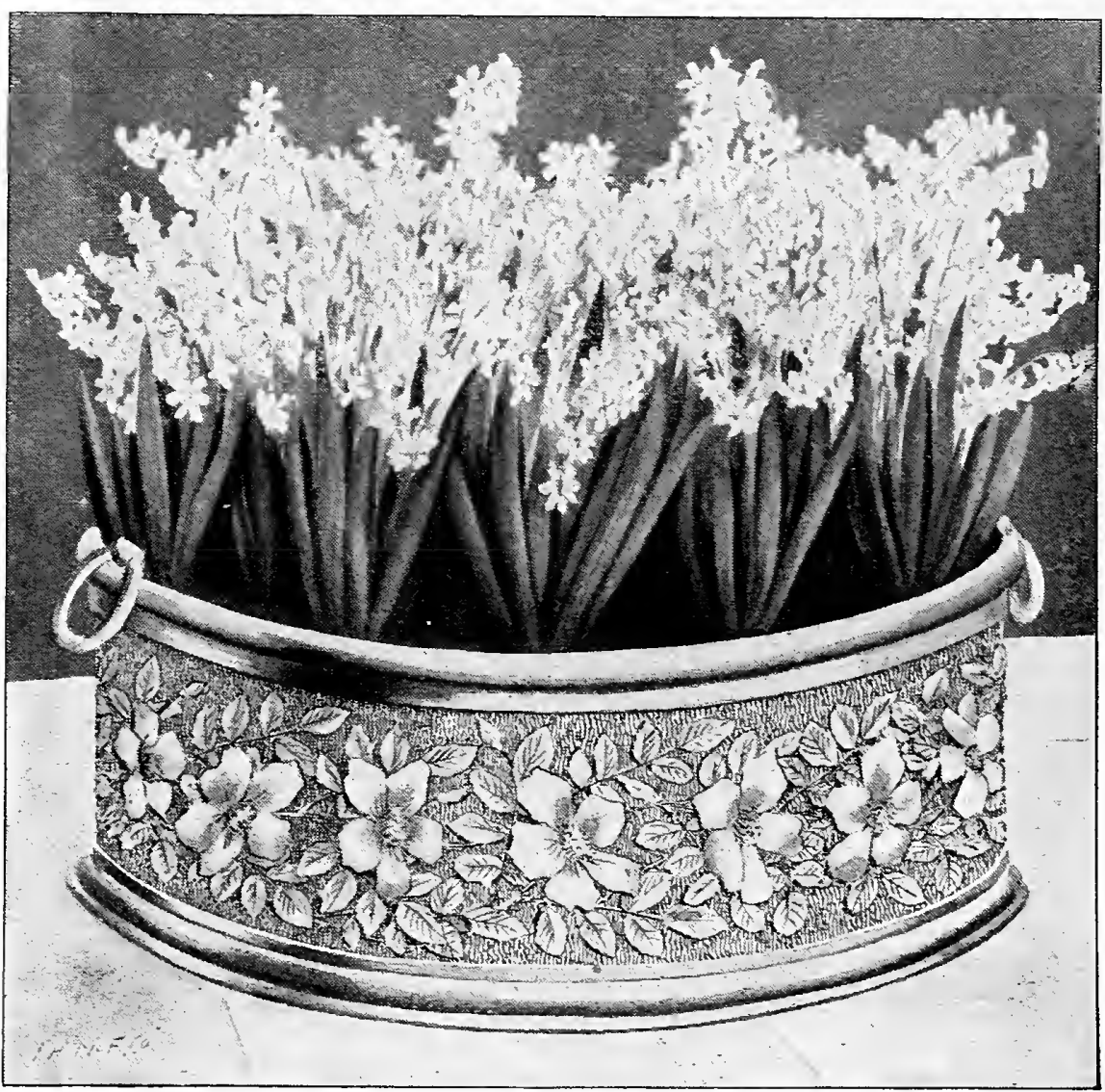

FERN DISH OF WHITE ROMAN HYACINTHS.

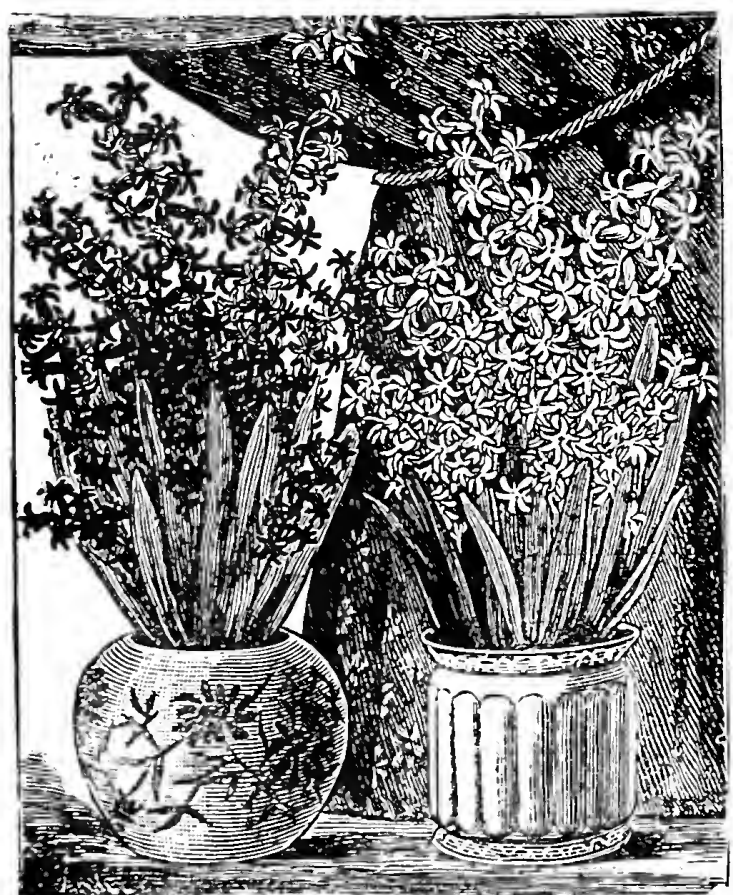

ROMAN HYACINTHS IN POTS. six spikes to each bulb, are rery low-priced, and are the eusiest bulbs to bloom in the house. The white variety is the earliest, most beautiful and satisfactory one to grow. By Dutch Hyacinths are meant the large-flowered varieties, which ererybody knows. The single varieties are the only ones to grow in the house, and are much more beautiful than the double sorts. Dutch Hyacinths should be treated in the same manner as Roman Hyacinths, except that the bulbs should not be brought out of the cellar before Februars 1. Single Early Tulips and Narcissi (Daffodils) can also be bloomed successfully in the house, if not attempted too early. They can be bloomed in March, and a splendid display of them can be readily had for Easter. They should not be brought from the cellar until March 1. The following varieties of Narcissi are the most satisfactory for blooming in the house: Bicolor Empress, Emperor, Incomparabilis Alba Stella, Von Sion, Poeticus, Ornatus, Barri Conspicuus, Golden Spur, Princeps, Paper White, and all varieties of Polyanthus Narcissi. Almost erery house contains flower pots, jardinières, etc., that might be utilized for growing bulbs. Zinc pans or bowls to fit inside of them can be made by any tinner, and the choicest ware thus used without injury.

\section{Collections of Bulbs Suitable for the House}

Prices of Dutch and Roman Hyacinths will be found elsewliere in this list, but I shall be pleased to make up collections suit able for the house at $\$ 2.50, \$ 5, \$ 10, \$ 15, \$ 20$ and $\$ 25$ each. These collections can be relied upon to be entirely satisfactory, both as to quality and price.

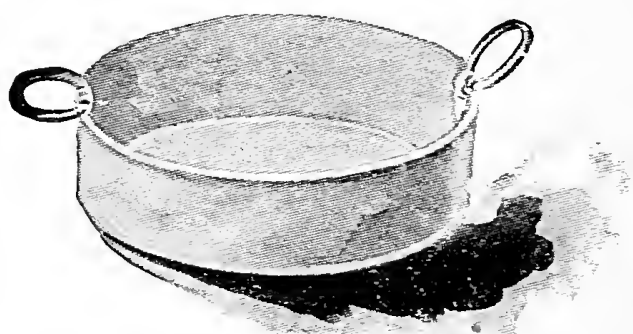

ZINC PAN FOR FERN DISH 


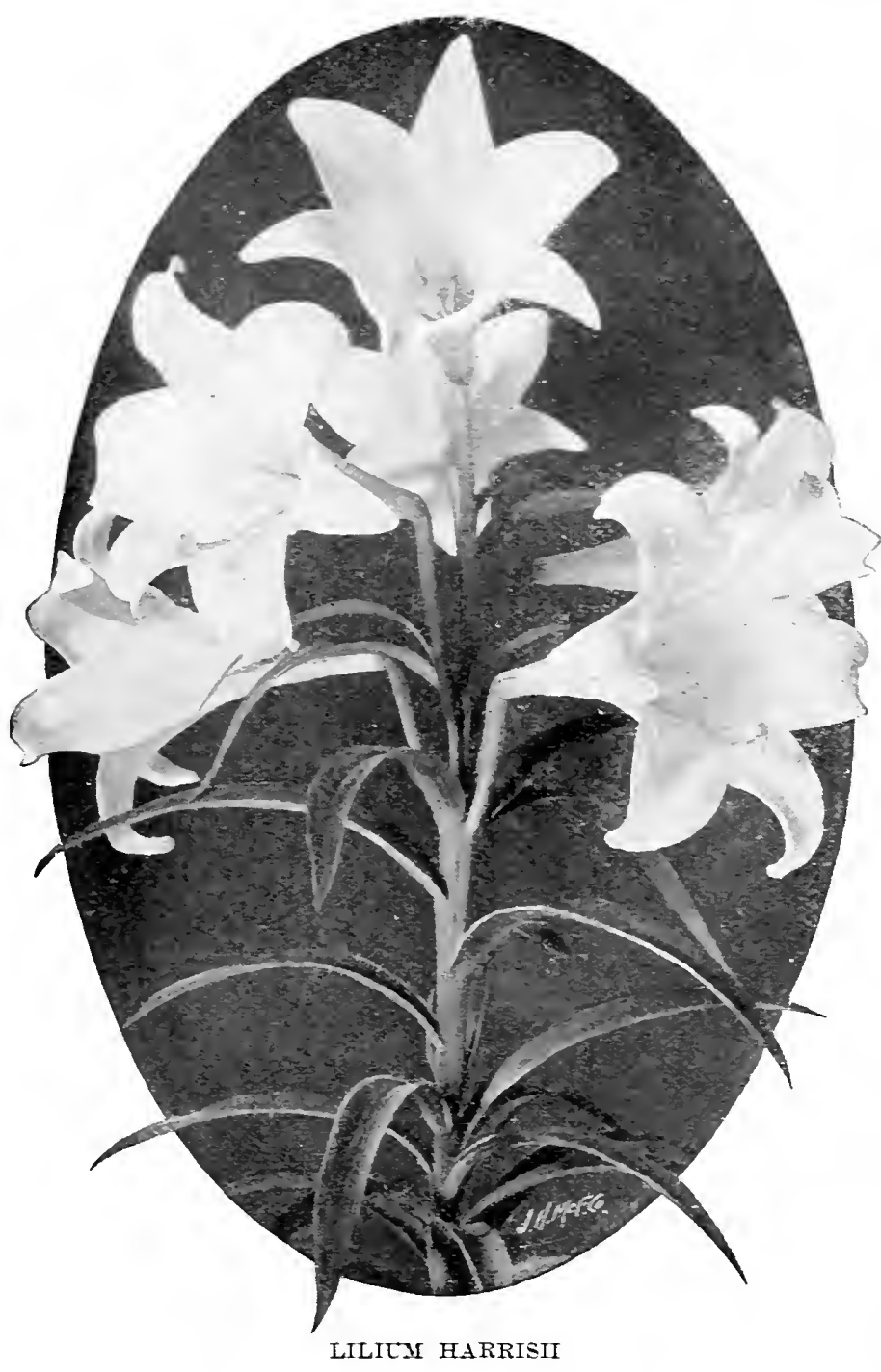

\section{Lilium Harrisii (True Bermuda Easter Lilies) (READY IN AUGUST)}

It is impossible to obtain Bermuda Lily bulbs that are entirely free from disease. We get the best bulbs obtainable, but cannot guarantee results

This Lily is now so well known that it needs no description. It is undoubtedly one of the most popular flowes erer used for forcing. The bulbs we supply are of the highest quality obtainable. and are perfectly true. and not mised with Lilium longiflorum. It cannot be grown outdoors unless extrenely well protected in winter. If a Lily of this character is desired for outdoor planting, L. Tongiflorum should be used.

Prices for orders booked vefore July I:

Bulbs 5 to $\tau$ inches in circumference ......................... Per doz. $\quad 100$

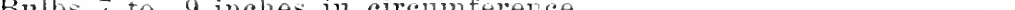

Bulbs 9 to 11 inches in circumference..................... $400 \quad 2500$

\section{Lilium Candidum}

(Ready in September and delivered as soon as ready)

Selected French bulbs, field-grown.......................... $\$ 100$ American bulbs, selected..................................... 1 501000

\section{Roman Hyacinths}

(Ready about September 1)

Prices for orders booked before Juiy I:

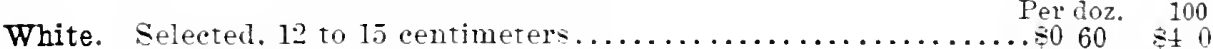
Extra-selected. 13 to 15 centimeters................... is 500

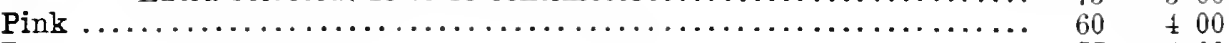
Blue................................................ 55

\section{Lily-of-the-Valley}

Extra-selected German pips. Reads latter part of Norember........ Per $100 \quad 1.000$ Americau pips. Reads iu Octuber............................ $150 \quad 900$ These pips are excellent for establishing plantations cutdoors as well as for forcing. The Germau pips are not ready in time for outdoor planting in the fall.

\section{Freesia refracta alba}

This charming fiotrer is one of the most satisfactor? bulbs that can be forced. It is certain to flower eitler in the conservators or house.

Laroe bulbs.............. Per doz. $100 \quad 1,000$ Nammoth bulbs........... $35 \quad 200 \quad 1500$

\section{Milla uniflora}

\section{(TRITELEIA)}

This charuing spring-flowering bulb is almost unknowu iu this countrs, but I know of nothing lorelier. I first saw it in the garden of Mr. Charles A. Daua. "Dosoris," Long Island, and was delighted, as I am sure erers one will be that tries it. It likes rather a moist soil and should be planted where it cau remain permauently. It can also be bloomed in the house with the same treatment as hracinths. For this purpose sereral bulbs should be planted in a 6- or 7 -inch pot.

Uniflora. Charming white, star-like flowers, with beautiful foliage which corers the ground like a carpet. One of the choicest hards bulbs. Should be used freels in erers garden. 20 ets, per doz. 75 cts per 100 . per 1,000

Uniflora violacea. Same as abore, with riolet flowers. 25 ct. per doz., $\$ 1$ per $100, \leqslant 5$ per 1.000 .

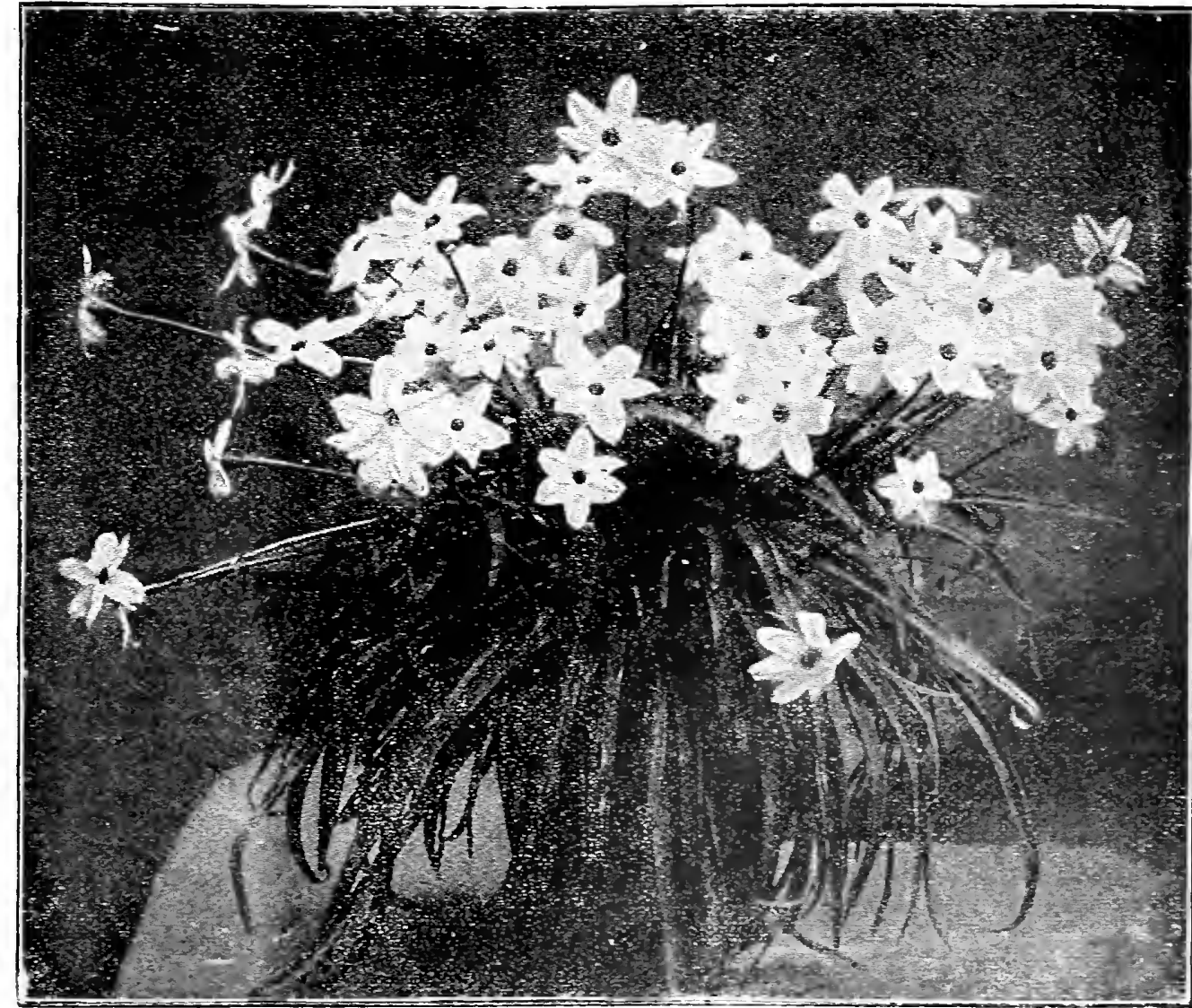

MILLA TXIFLORA GROWI IN A POT 


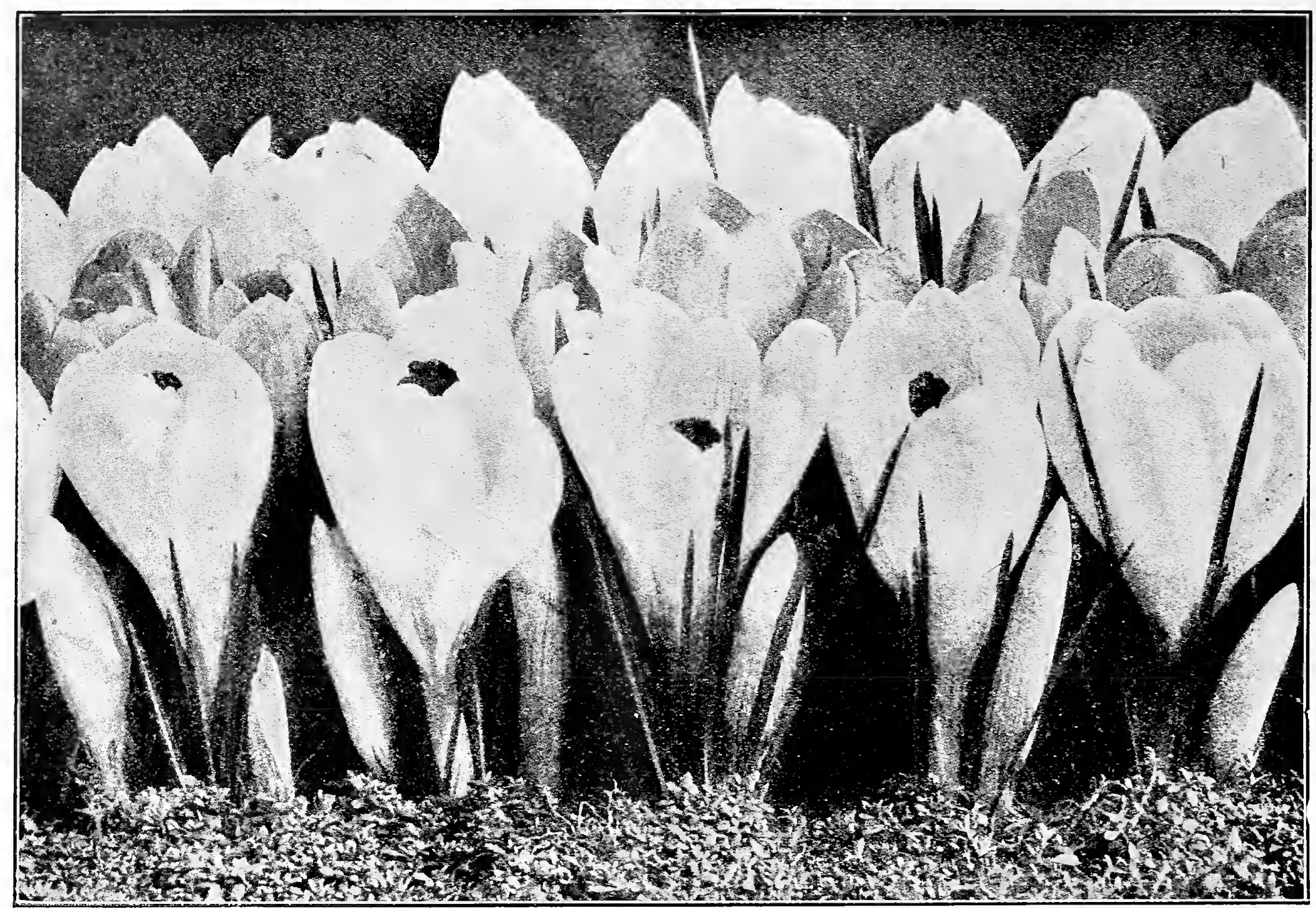

LARGE-FLOWERED CROCUSES

\section{CROCUSES IN THE GRASS}

There is no reason why everybody, even if the possessor of only the smallest strip of grass, should not have it filled with Crocuses. The cost is but a trifle, and once planted they are no further trouble, and bloom beiutifully year after year and do not interfere with the mowing or care of the lawn; but grass should not be mown for two weeks after flowering of Crocuses, and it does not need mowing earlier than this. They should be planted in the fall and set from 1 to 2 inches below the surface of the ground; they can be planted with a narrow trowel or stiff-bladed knife. Mowing the grass in the spring after the Crocuses are done blooming will not injure them in the least.

The Crocus is the first flower to bloom in the spring, and it is no unusual sight to see the ground covered with snow and the Crocus in bloom at the same time. They are entirely hardy, do not deteriorate, and may be left undisturbed for years. It is a good thing to plant tulip and hyacinth beds full of them, as they bloom and are out of the way before the other bulbs flower.

Carpet-beds with designs worked out with Crocus are very effective in carly spring. Crocuses should be planted $1 \frac{1}{2}$ to 2 inches apart, and for this purpose Mixed Crocuses should never be used.

My advice would always be to plant Named Crocuses; they are very superior to Mixed sorts

SELECT NAMED CROCUS

Prices quoted are for orders booked by July 1, 1906. Not less than 50 of a kind will be supplied

Albion. Striped blue and white.............. Per $\frac{100}{75}$

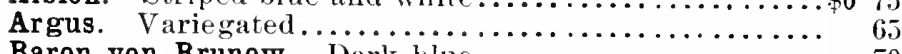

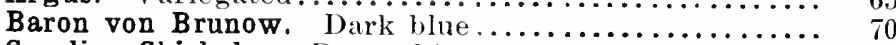

Caroline Chisholm. Pure wlite.................. 70

Cloth of Gold. First size; golden, striped brown...... 50

Cloth of Silver. First size; white, violet-striped....... 65

Grand Lilac. Lilac; very early and very tine..........

Grootvorst. White and violet.................. 70

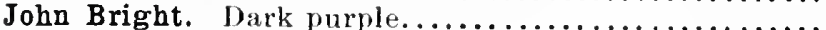

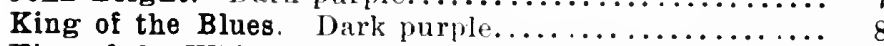

King of the Whites. Large; snow-white; splendid new

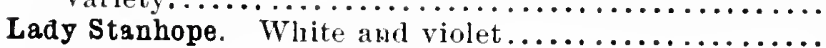

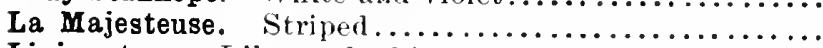

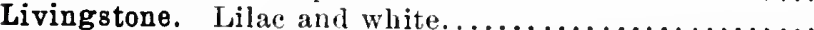

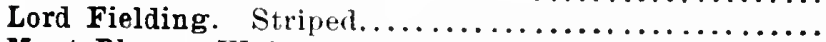

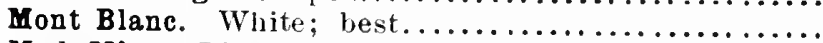

Mad. Mina, Light blue, striped; very early; extra fine.

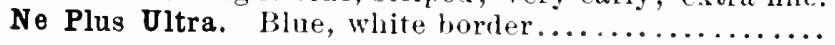

610

575

435

50

$\begin{array}{ll}6 & 00 \\ 6 & 50\end{array}$

$\begin{array}{ll}6 & 50 \\ 6 & 85\end{array}$

$\begin{array}{ll}7 & 75 \\ 5 & 75\end{array}$

575

5 3.

575

560

575

625

625
Prince Albert. Blue........................ Per $\frac{100}{75} \$ 0^{1,000}$

Purpurea grandiflora. Large, deep purple............ 85 . 75

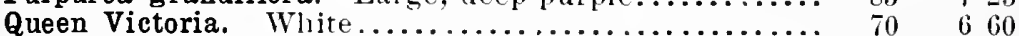

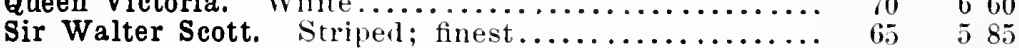

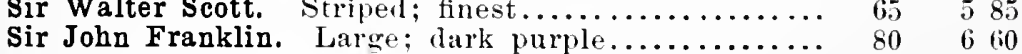

$\$ 6 \quad 10 \quad$ Sir John Franklin. Large; dark purple.............. $80 \quad 660$

785

\section{CROCUS IN SEPARATE COLORS}

Purple. One variety......................... Per $100 \quad \begin{gathered}1,000 \\ 50\end{gathered} \$ 435$

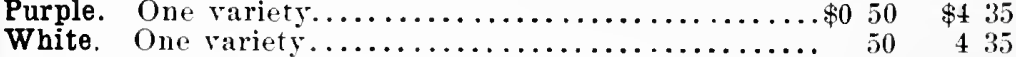

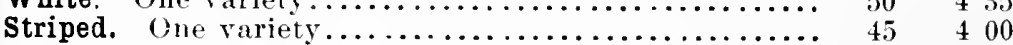

Large Yellow. Manmoth bulbs, first size.......... $85 \quad 750$

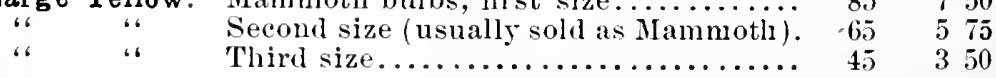

\section{MIXED CROCUS}

White

385

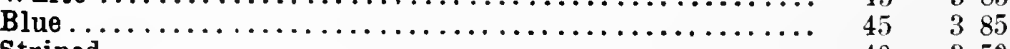

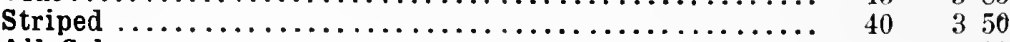

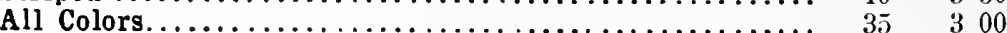




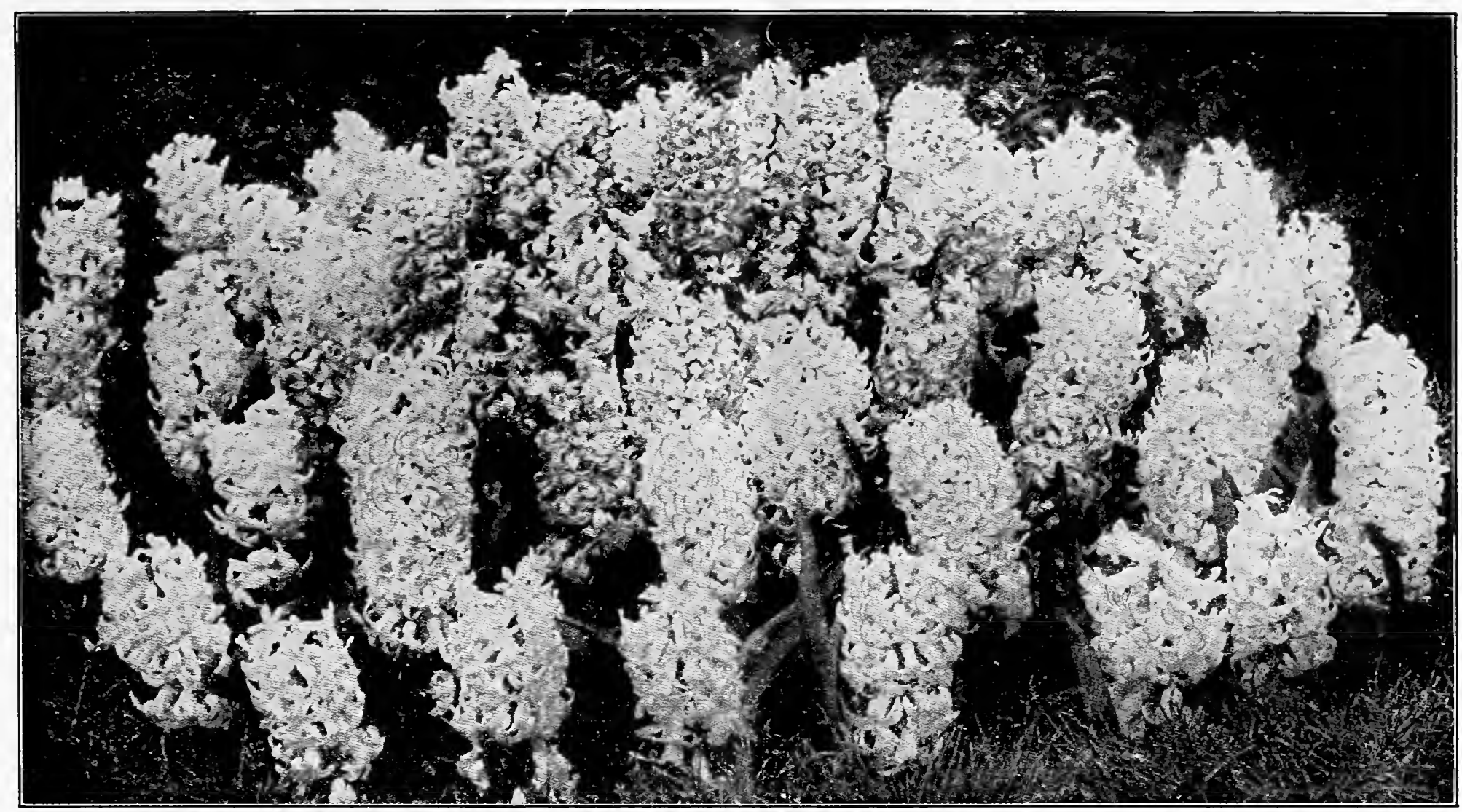

BED OF White Hyacinths, PlaNTed With BEDdiNG hyACINTHS IN SEPATATE COLORS

\section{HYACINTHS}

All prices on Hyaclnths are for orders booked by July I. Ready about October I

\section{MIXED HYACINTHS}

Mixed Hyacinths should never be used for house or greenhouse culture.

\section{SINGLE}

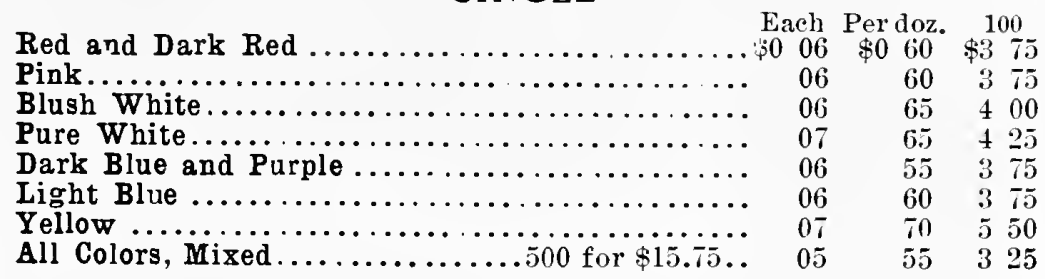

\section{DOUBLE}

Double Hyacinths are so inferior to singles that they should never be used for any purpose.

Red and Pink

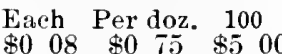
White.

Blue and Purple.

Yellow. ...

(ll

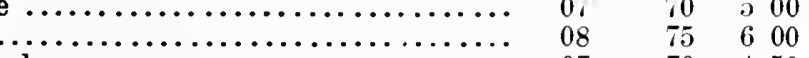

\section{BEDDING HYACINTHS}

Separate varieties without names. These are superior in quality to mixed Hyacinths, and very satisfactory for bedding, as uniformity in color and height can be had by their use.

\section{SINGLE}

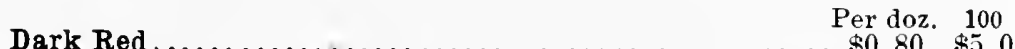

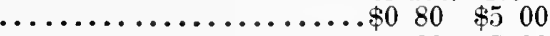

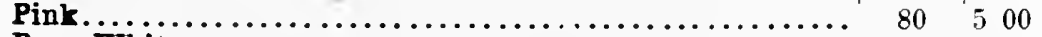

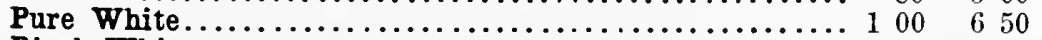

Blush White $\ldots \ldots \ldots \ldots \ldots \ldots \ldots \ldots \ldots \ldots \ldots \ldots \ldots \ldots \ldots \ldots \ldots \ldots \ldots$
Dark Blue $\ldots \ldots \ldots \ldots \ldots \ldots \ldots \ldots$

Light Blue

Yellow

\section{SECOND-SIZED NAMED HYACINTHS}

These second-sized Hyacinths will be found fine for forcing, and are highly recommended for those who desire extra good bulbs for bedding. They embrace the leading named sorts of this list, are larger bulbs than those sold as mixed, and strictly true to name and color, thus giving exact shades wanted. We can offer them in the following rarieties, all of which are described in the following list. We offer single varieties only, as double sorts are very inferior.

Red, Rose and Pink. Baron von Thuyll, General Pelissier, Gertrude, Gigantea, Roi des Belges, Lord MIacaulay, Moreno.

White and Blush White. Grande Blanclie, Grandeur à Merreille, Mr. Plimsol, Paix de l'Europe, Baroness von Thuyll.

Dark and Light Blue. Baron von Thuyll, Charles Dickens, Grand Maitre, King of the Blues, La Peyrouse, Marie, Regulus, Leonidas.

Prices: Any or all of the above varieties, 90 ets. per doz., $\$ 6$ per $100, \$ 55$ per 1,000 . Fifty will be supplied at the 100 rate and 500 at the 1,000 rate. These bulbs are equal to those usnally sold as first-sized in this country.

\section{SINGLE SMALL HYACINTHS FOR FORCING}

These single Hyacinths, planted on arrival from Holland, may be had in flower early in January. The bulbs and flowers of these small Hyacinths are about the same size as Roman Hyacinths, but much stronger, and the bells are larger, while they do not cost half the price of Romans. Only these varieties which produce the fine flowers and force easily are selected for this purpose. They may be planted like tulips, in pots or boxes close together. Last season we sold large quantities, and our customers inform us that they are a great acquisition to suceeed the Roman Hyacinths, blooming from January till April.

Per doz. 100

(n.....................................\$0 40 \$2 50

Pink....................................... $40 \quad 250$

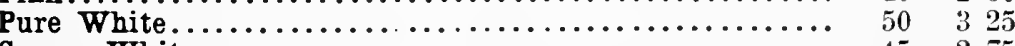

Cream White.................................... $45{ }_{4}^{2} 75$

Dark Blue..................................... $40 \quad 250$

All Colors, Mixed....................................... 


\section{Single Named Hyacinths}

To secure the 100 price on named Hyacinths, not less than 25 bulbs of a variety can be ordered

Named Hyacinths of the quality imported by us can not be obtained in this country. They are selected exhibition bulbs and have won first prizes wherever exhibited, both in this country and in Europe. Hundreds of our customers write us that they have never seen such fine flowers as these bulbs produce. We are confident that any one who will try these Hyacinths, or any of the named bulbs offered in this Catalogue, will never again be satisfied with the inferior bulbs sold by dealers in this country. Varieties marked with an asterisk $\left({ }^{*}\right)$ are very desirable.

\section{SINGLE DARK RED}

\begin{tabular}{|c|c|c|c|}
\hline armine; fine spike............ & $\begin{array}{l}\text { Each } \\
\$ 015\end{array}$ & $\begin{array}{l}\text { Per doz. } \\
\quad \$ 150\end{array}$ & $\stackrel{100}{\$ 950}$ \\
\hline osmos. Dark rose; large spike......... & 15 & 150 & 1000 \\
\hline Fiance Royal. Bright rose; large truss........ & 15 & 150 & 925 \\
\hline eneral Pelissier. Deep & 12 & 130 & 850 \\
\hline ertrude. Red; large, fine spike....... & 12 & 120 & 850 \\
\hline 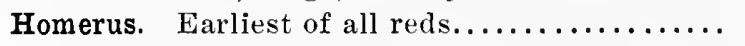 & 20 & 200 & 1100 \\
\hline ncomparable. Brilliant sc & 15 & 140 & 1000 \\
\hline Josephine. Brilliant & 12 & 120 & 850 \\
\hline 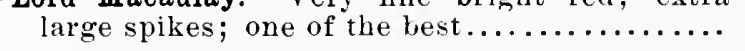 & 13 & 135 & 850 \\
\hline Prima Donna. Lustrous color ... & 20 & 220 & 1375 \\
\hline Queen Victoria Alexandra. Dark carmine ...... & 20 & 200 & 1275 \\
\hline & 20 & 200 & 1275 \\
\hline Robert Steiger, Deep crimson............. & 10 & 110 & 800 \\
\hline $\begin{array}{l}\text { oi des Belges. Ex } \\
\text { extra large spike.. }\end{array}$ & 10 & 110 & 800 \\
\hline Veronica. Dark red & 15 & 150 & 925 \\
\hline Von Schiller. Deep & 15 & 160 & 1160 \\
\hline William the First. Brilli & $1 \overline{0}$ & & 1050 \\
\hline
\end{tabular}

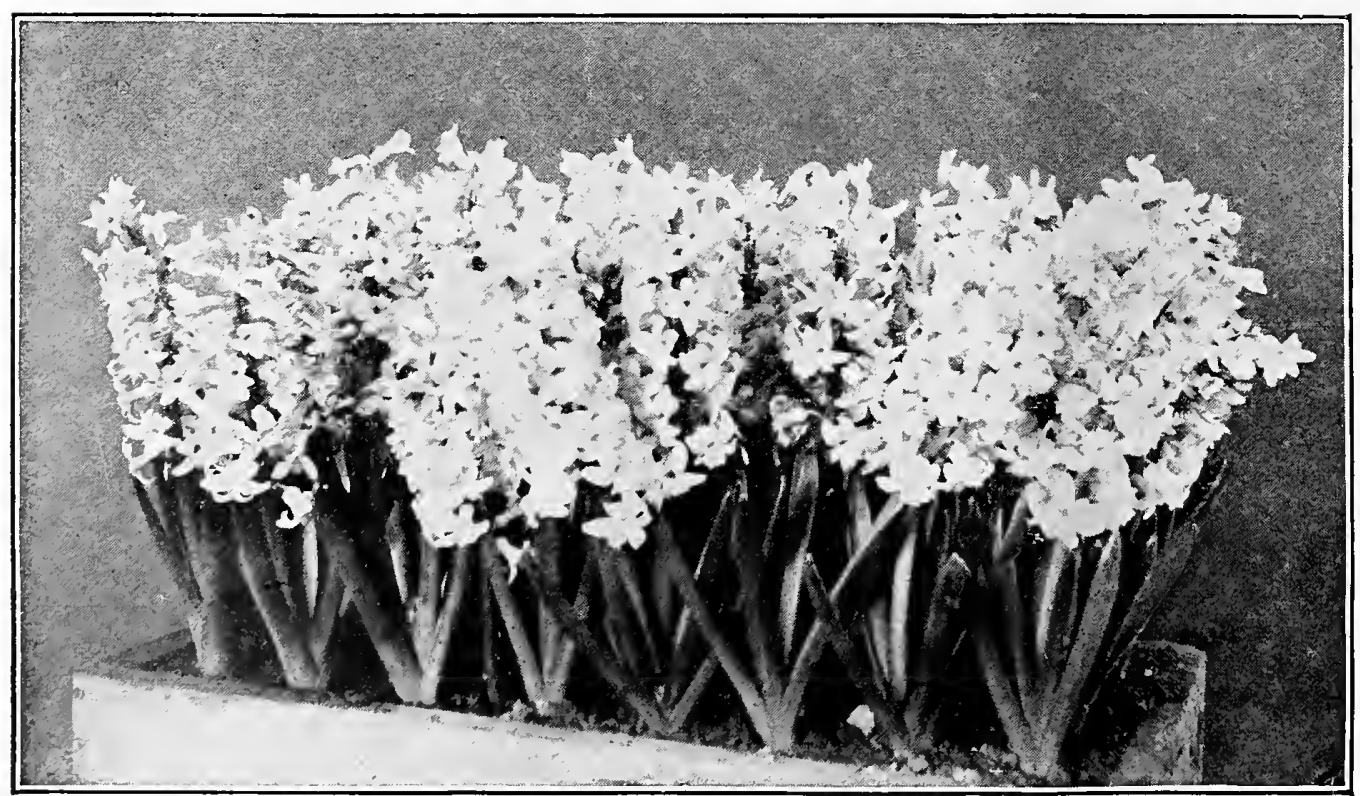

CZAR PETER HYACINTHS

\section{SINGLE ROSE}

*Baron von Thuyll. Very fine pink; extra large spike.................................... $13 \quad \$ 140 \$ 875$ * Cavaignac. Extra fine salmon-pink; distinct and splendid color; large, full spike............. Cardinal Wiseman. Soft rose, carmine striped;

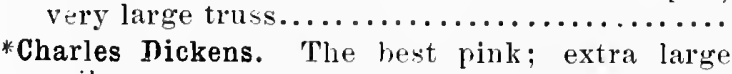

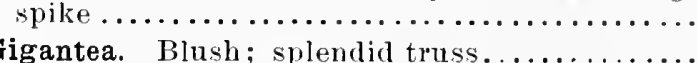

Grand Vainqueur. Fine pink; tall spike; very

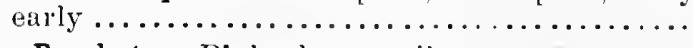

Le Prophete. Pink; large spike.............

Lord Percy. Fine soft rose; new...........

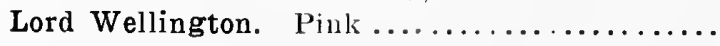

Madam Hodson. Pink, carmine-striped........

* Maria Cornelia. Light pink; earliest of them all. $\begin{array}{lllll}20 & 2 & 25 & 13 & 75\end{array}$

$\begin{array}{lllll}15 & 1 & 60 & 10 & 50\end{array}$

$15 \quad 150 \quad 1050$

$12 \quad 125 \quad 8 \quad 40$

$13 \quad 150 \quad 950$

$12 \quad 125 \quad 850$

$\begin{array}{lllll}25 & 2 & 40 & 15 & 00\end{array}$

$15 \quad 150 \quad 1050$

$\begin{array}{lllll}15 & 160 & 11 & 75\end{array}$
$12 \quad 125 \quad 850$

Moreno. Very fine pink; extra large spike; very

early; splendid sort.................. $\$ 0$ i6 \$1 $75 \$ \$ 1050$

Norma. Satiny pink................... $14 \quad 150 \quad 1050$

* Rosea Maxima. Early, delicate rose........ $13 \quad 150 \quad 950$

Sarah Bernhardt. Bright rosy pink, delicious

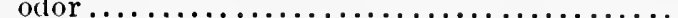

$\begin{array}{rrrrr}15 & 1 & 60 & 10 & 50 \\ 14 & 1 & 50 & 9 & 00\end{array}$

\section{SINGLE DARK BLLE}

Argus. Blue, white eye; extra ...........

Baron von Thuyll. Large truss.............

Bleu Mourant. Dark blue...............

*Charles Dickens. Porcelain-blue; large; extra

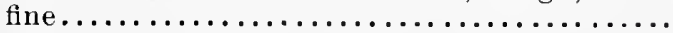

*King of the Blues. Splendid; finest dark blue.

Leopold II. Fine blue; large truss; splendid Hower.............................

Marie. Rich purple and indigo; enormous spike. Mimosa. Bluish purple; splendid ...........

Pieneman. Dark porcelain-blue; very large

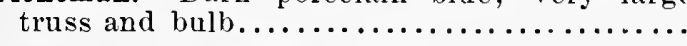

Sir Henry Barclay. Black. William the First. Large spike; early..........

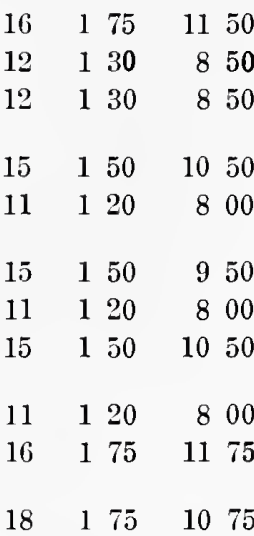

\section{SINGLE LIGHT BLUE}

*Blondin. Silvery tinged Each Per doz. 100 blue; fine truss.......\$0 $13 \quad \$ 130 \$ 850$

* Captain Boynton. Azureblue; large spike.....

* Czar Peter. Porcelainlilac; grand spike....

*Enchantress. Clear light blue; one of the finest blues; extra fine spike. An exhibition variety..

* Grand Lilas. Azure-blue; perfect spike.........

*Grand Maitre. Very large spike................

La Peyrouse. Clear blue.

Leonidas. Splendid large truss ................

Lord Byron. Azure-blue; very large spike......

*Lord Derby. One of the very finest............

*Lord Palmerston. Light blue, white eye...... * Queen of the Blues. Light blue, witl silvery appearance; extra fine.................

*Potgieter. Light blue.................

Regulus. Light blue, with dark stripes.......

\section{SINGLE WHITE}

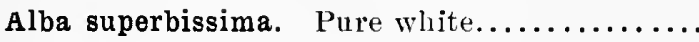
*Albertine. Pure white; early, large spike ;

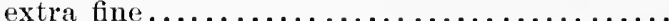

* Baroness von Thuyll. Early, pure white...... Blanchard. Pure white; very large spike...... Blancheur à Merveille. Pure white; very fine

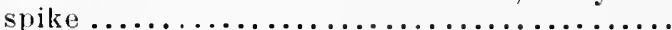

* British Queen. Pure white; new; extra fine... * Grande Blanche. Blush white; large bells and

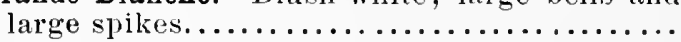




\section{SINGLE WHITE HYACINTHS, continued} Crown Princess. Pure white; fine,

* Grandeur à Merveille. Rosy blush.. $13 \quad 125$

La Franchise. Waxy white; large

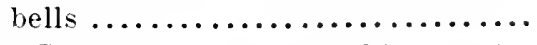

* La Grandesse. Purest white; extra fine; best of all whites..........

La Neige. Superb; very early;

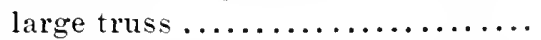

Leviathan. Beautiful blush; large bells and spike; a most distinet and charming variety............

L'Innocence. Fine white; extra fine and large truss.................

*Mad. Vanderhoop. Pure white;

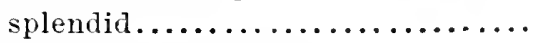

Mammoth. Blush white...........

Mary stuart. Fine, pure white.....

Mina. Pure white; fine spike.......

* Mont Blanc. Fine pure white; grand spike ...........................
Mr. Plimsol. Irory-white; large bells

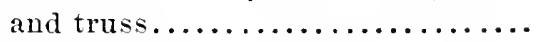

* Paix de l'Europe. Very fine; snowwhite; very large truss.......... Pavillon Blanc. Pure white........ Queen Victoria. Pure white....... Snowball, Pure white ............

Voltaire. Creamy white ............

\section{SINGLE YELLOW}

Australian Gold. Golden yellow; fine spike........................ splendid truss.................

Hermann. Orange-yellow..........

*Ida. Citron-yellow; large spike; one of the finest yellows in cultivation.

King of Holland. Orange-yellow....

* King of the Yellows. Fine bright yellow; grand spike...............
La Citroniere, Bright citron-yellow; La Citroniere. Bright citron-yellow; fine spike .......................

La Pluie d'Or. Pale yellow .......... large spike..................

Obelisk. Splendid pure yellow; one

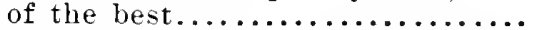

*Yellow Hammer. Golden yellow; extra fine truss.................
$15 \quad 150 \quad 925$

$20 \quad 2 \quad 10 \quad 1350$

$15 \quad 160 \quad 10 \quad 25$

$20 \quad 2 \quad 10 \quad 1325$

$15165 \quad 1025$

$20 \quad 2 \quad 00 \quad 1200$

$\begin{array}{lllll}20 & 2 & 00 & 12 & 50\end{array}$

$15 \quad 160 \quad 1050$

$25 \quad 240 \quad 1475$

$15 \quad 150 \quad 950$

$13 \quad 125 \quad 850$

$20 \quad 2 \quad 00 \quad 1135$

$20 \quad 200 \quad 1250$

$25 \quad 2 \quad 75 \quad 1875$

$13 \quad 130 \quad 850$

$25 \quad 2 \quad 25 \quad 1450$

$22 \quad 250 \quad 14 \quad 25$

$15 \quad 150 \quad 1000$

$17 \quad 185 \quad 1250$

$16 \quad 180 \quad 1250$

$20 \quad 2 \quad 00 \quad 1350$

$20 \quad 200 \quad 1175$

$14 \quad 130 \quad 925$

$\begin{array}{lllll}16 & 1 & 15 & 13 & 00\end{array}$

$23 \quad 2 \quad 40 \quad 1450$

$20 \quad 2 \quad 10 \quad 1575$ $\begin{array}{llll}20 & 2 & 10 & 13\end{array}$

\section{SINGLE VIOLET AND LILAC}

Charles Dickens, Large dark lilac; Each Perdoz. 100 splendid ...................\$0 25 \$2 40 \$1500 Distinction. Splendid maroon; new. $\begin{array}{lllll}60 & 6 & 00 & 39 & 0 .\end{array}$ Haydn. Brilliant dark plum ....... $18 \quad 200 \quad 1200$ Jeschko. Lilac, striped with rioletred...........................

Lord Mayo. Rich purple-blue, with white ere...................

Lord Balfour. Violet ............. Sir Edward Landseer. Dark riolet..
Sir Henry Havelock. Plum or purpleSir Henry Havelock. Plum or purple-

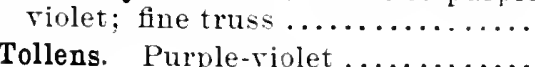
$\begin{array}{lllll}18 & 2 & 00 & 12 & 00\end{array}$ $25 \quad 250 \quad 1500$ $182200 \quad 1200$ $\begin{array}{llllll}20 & 2 & 00 & 12 & 75\end{array}$

\section{ENGLISH DAISIES}

The can supply Donble English Daisies for naturalizing at 50 ets. per doz., \$3.25 per 100 , $\$ 30$ per 1,000 .

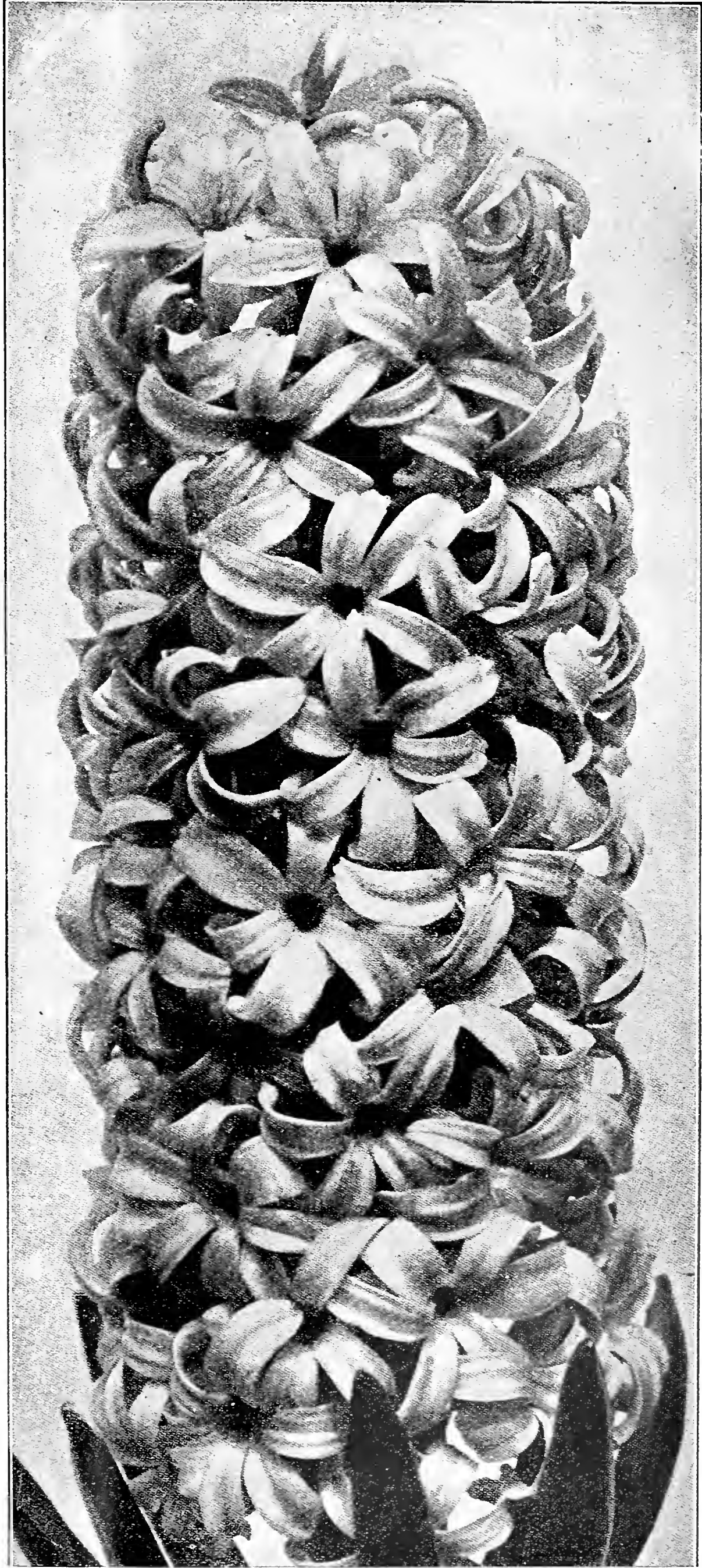

IORD BSROS. Character of Hower produced by our first-sized Named Hyacinths 


\section{Double Named Hyacinths}

Double Named Hyacinths are inferior to the Single varieties in every respect

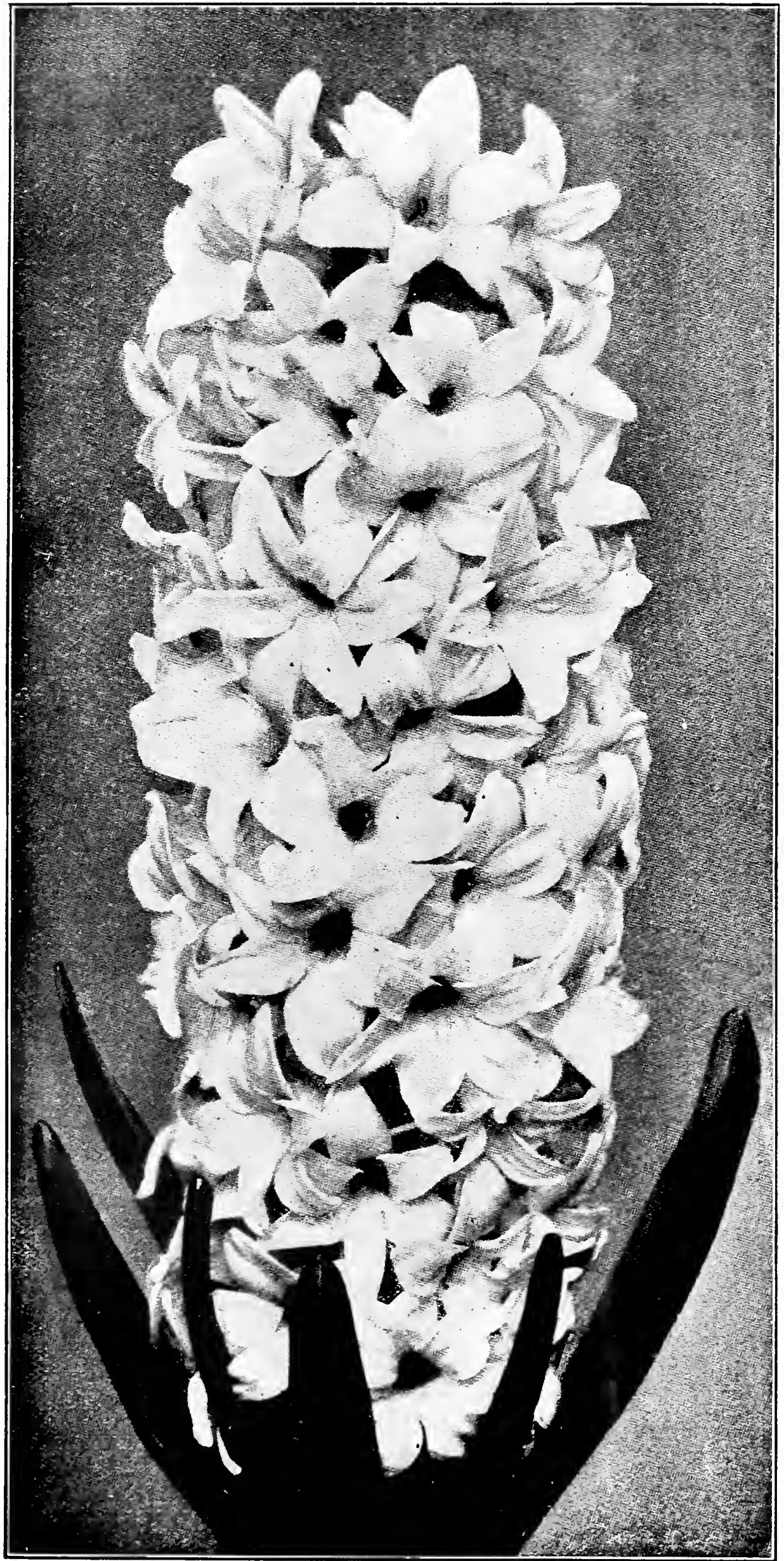

LA GRANDESSE. Character of flower produced by our first-sized named bulbs
DOUBLE DARK RED

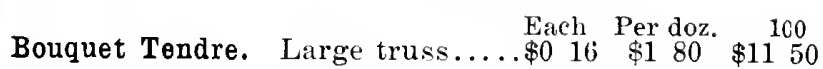
Noble par Merite. Splendid truss. $\begin{array}{lllll}14 & 1 & 30 & 8 & 50\end{array}$ Princess Royal. Fine dark center. $\begin{array}{lllll}13 & 1 & 45 & 9 & 00\end{array}$ Regina Victoria. Dark rose.... $16 \quad 16 \quad 75 \quad 1150$

\section{DOUBLE ROSE}

Bouquet Royal. Rosy salmon.... $14 \quad 14 \quad 130 \quad 840$ Grootvorst. Fine rose; good truss. $14 \quad \begin{array}{llll}3 & 30 & 8 & 25\end{array}$ Lord Wellington. Best double pink. $\quad 20 \quad 200 \quad 1350$

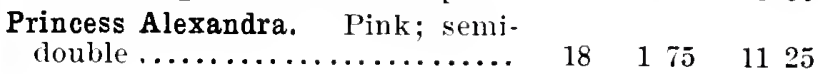

\section{DOUBLE DARK BLUE}

Garrick. Lilac-blue; splendid....\$0 $12 \quad 135 \quad 850$ Lord Raglan. Azure-blue, dark

center..................... $12 \quad 13 \overline{5} \quad 850$

Prince of Saxe Weimar. Semi-

double ..................... $16 \quad 16 \quad 15 \quad 1050$

\section{DOUBLE LIGHT BLUE}

Blocksberg. Extra fine truss...... $\$ \begin{array}{llllll} & 16 & 1 & 75 & 10 & 50\end{array}$ Charles Dickens. Light blue; extra

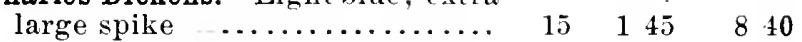

Mignon de Dryfhout. Semi-double. $15 \quad 145 \quad 875$

Van Speyk. Fine porcelain-blue;

large spike and extra-large bells. $12 \quad \begin{array}{llll}12 & 35 & 850\end{array}$

\section{DOUBLE WHITE}

Bouquet Royal. Pure white...... $15 \quad 150 \quad 950$

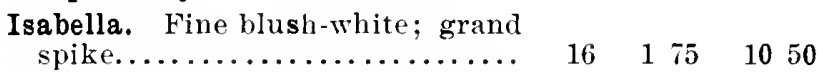

La Tour d'Auvergne. Pure white; $15 \quad 150 \quad 9-5$ $\begin{array}{rrrrrr}\text { large truss................... } & 15 & 1 & 50 & 9 & 75 \\ \text { La Virginite. Rosy center...... } & 14 & 120 & 8 & 20\end{array}$

\section{DOUBLE YELLOW}

Bouquet d'Orange............... $16 \quad 175 \quad 1150$ Goethe. Splendid pale yellow..... $15 \quad 150 \quad 1000$ Ophir d'0r. Reddish purple center. $\quad 17 \quad 160 \quad 1050$ William III. Extra fine apricot.. $17 \quad 1760 \quad 1125$

\section{Azalea Indica}

(For Greenhouse Culture)

Buyers desiring Azaleas in quantity or large specimens should write for special prices.

\section{PRICES :}

Strong plants, with heads 9 across......................\$6 $50 \quad \$ 1000$ Strong plants, with heads 10 to 12 inches

across..................... $750 \quad 4750$

Strong plants, with heads 12 to 15 inches

across........................ $1000 \quad 7500$

Stroug plants, with heads 18 to 24 inches

across................each, $\$ 3.50$.

\section{Those prices hold good until October I}

Large specimen plants from $\$ 3$ to $\$ 10$ each and upward.

Delivery will be made early in November, as soon as importations are received.

We can supply all the best sorts. Azaleas furnished by us have given unqualified satisfaction. 


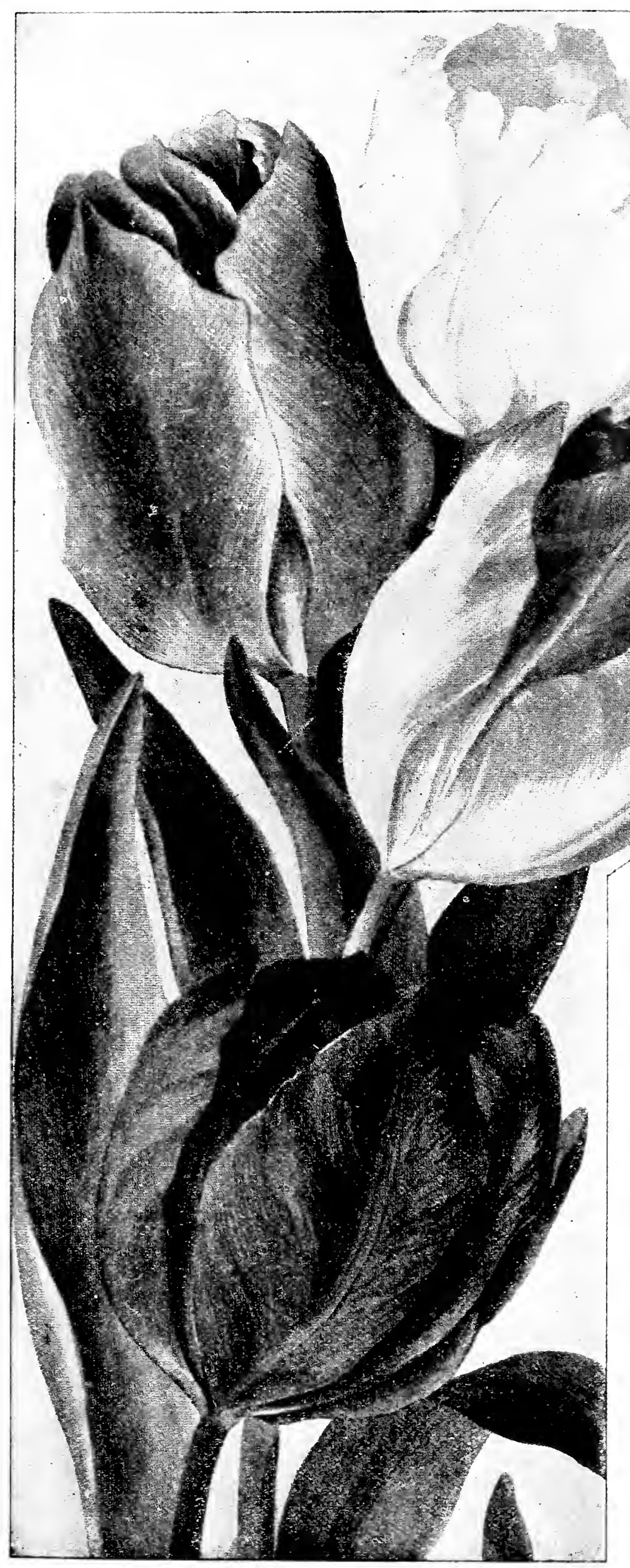

\section{TULIPS}

We have made a special effort to get the best Tulips grown in Holland, and to supply our customers with the superior class of bulbs usually monopolized by the highclass trade in England. We have been quite successful in this, and, if so disposed, could publish hundreds of enthusiastic letters received from our customers, testifying to the surpassing quality of the Tulips imported for them. These letters, of course, refer to Named Tulips. The best results can not be expected when the cheap Mixed Tulips are planted. Yet our customers report most satisfactory results from our cheapest Mixed Tulips, which are sold at the low price of $\$ 8.50$ per 1,000 , and which are very superior to Mixed Tulips usually sold in this country. For our own planting, however, we never use mixed bulbs of any description. We think fewer bulbs of the highest grade give more pleasure and satisfaction than many of an inferior quality. 


\section{TULIPS IN MIXTURE}

\section{Prices quoted are for orders booked by July I, I906. Special prices quoted for 10,000}

Fine Single Mixed. Quality superior to that generally

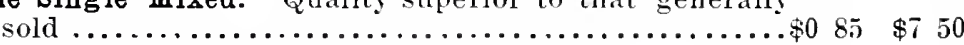

Extra Fine Single Mixed. Extra selected........... $110 \quad 900$

Fine Double Mixed. Quality superior to that generally

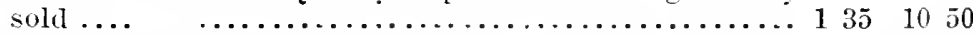

Extra Fine Double Mixed. Extra selected........... $150 \quad 1200$

Extra Fine Late Double Mixed .................. 1 25 1000

\section{SINGLE NAMED EARLY TULIPS}

If best results are desired, Named Tulips should be used. Mixed Tulips are not recommended, although the mixtures imported by us are very superior to those usually sent out.

The letters A, B and $C$ indicate the proportional earliness of the rarieties if planted outdoors. A and B may be used together for bedding purposes, but those marked $\mathrm{C}$ are later. The numbers preceding names show the height of growth in inches. Those marked $\mathrm{F}$ after the name are the best for forcing in the house or greenhouse.

Prices quoted are for orders booked by July 1, 1906 :

Albion. The finest pure white Tulip in cul- $\begin{array}{lll}\text { Per doz. } & 100 & 1,000\end{array}$

tivation.........................\$0 $45 \$ \$ 300 \$ 2100$

B 7 Arms of Leyden. IVnite and red........ 25 $140 \quad 1050$

B 7 Artus. Brilliant red. F............. $30 \quad 185 \quad 1350$

B 7 Adeline. Rosy crimson; fine......... 35 2 10

B $T$ Belle Alliance. Scarlet; rery fine. F.... $45 \quad 325 \quad 2550$

B 6 Bride of Haarlem, Silver. Pule white. feathered with bright cherry-red........ $90 \quad 550$

B 6 Bride of Haarlem, Golden. Golden yellow, feathered with bright red ............. $125 \quad 750$

B 6 Brutus. Orange-crimson ............ 25 $170 \quad 1150$ A 8 Canary Bird. Fine clear yellow. F...... $30 \quad 2 \quad 00 \quad 1600$

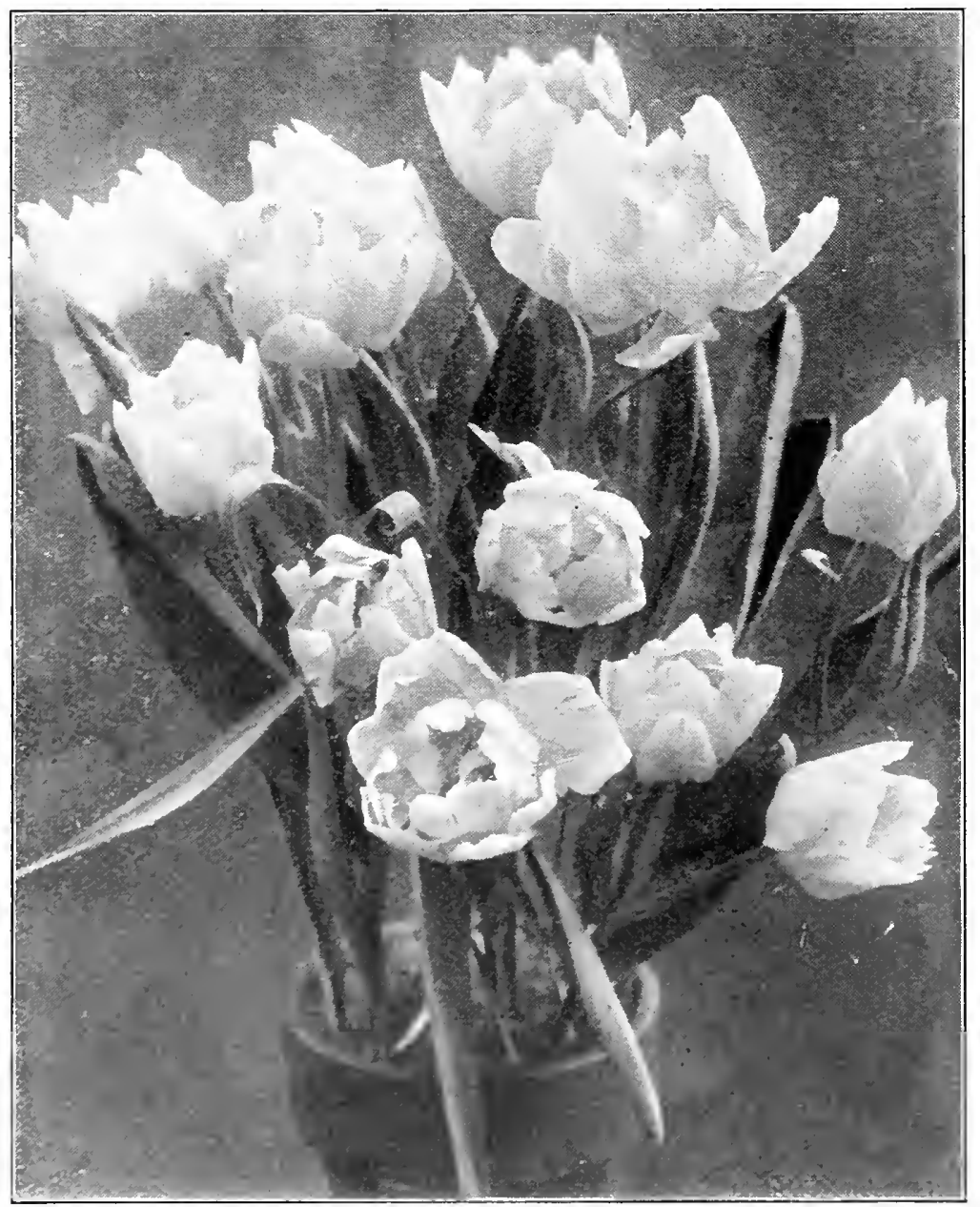

DOCBLE TCLIP, MUKHLL Bloomed in the house)
8 Chrysolora. Laroe ger doz. 100 low for outdoors. F..............\$0 20 \$1 25

C 9 Couleur Cardinal. Cardinal-red; one of the

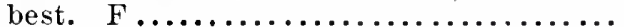

B 8 Couleur Ponceau. White ground, with rosy

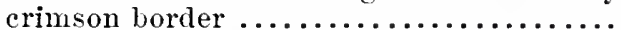

B 6 Cottage Maid. White, edged with rose: extra fine, lovely; splendid for bedding. F. Cramoisie Brilliant. Bright rermilion, large Hower; fine for forcing...............

B 7 Crimson King. Fine red. F...........

A 8 Duc d'Orange. Fellow and orange .......

A 6 Duc de Berlin. True. Red and yellow; early.

A 6 Duc van Thol, Red and Yellow...........

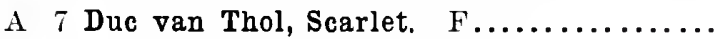

A 6 Duc van Thol, Yellow.

A 6 Duc van Thol, White.

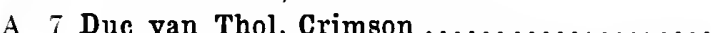
Duc van Thol, White Maximus. The best white Tulip for very early forcing........ Duc ran Thol Tulips are small and rery early. We do not consider them rery satisfactory, either for forcing or outroor planting.

B 9 Duchesse de Parma. Brownish red, yellow stripe; splendid bedder. Special low

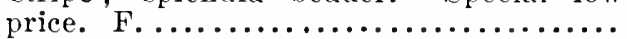

B 9 Duchess of Austria. Large; orange, red

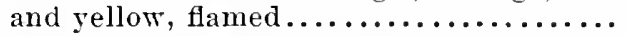

B 8 Eleonore. Dark violet, white-edged .......

A 7 Fabiola (Grand Duc de Russie). White, deep violet-striped; very fine ...........

B 7 Gold Finch. Golden yellow, sweet.scented;

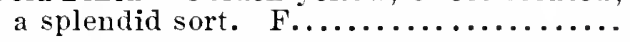

B 7 Grandmaster of Malta. Red and white.... Jacoba van Beyeren (White Swan). Pure

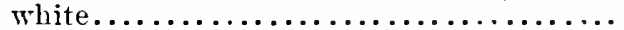

B 8 Joost van Vondel. Large; cherry-red and

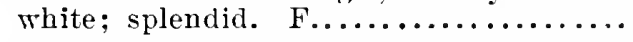

Joost van Vondel, White. Silvery white; magnificent flower; the fimest...........

B 9 Keizerkroon. Red and gold; large and fine.

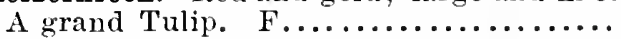

B 8 King of Yellows. Deep golden vellow.....

B 9 La Reine (Queen Victoria). Rosy white.

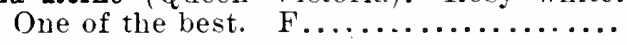

B 9 La Remarkable. Pure riolet, white edge..

B 9 L'Immaculee. Pure white; fine. F......

B 8 Leonardo da Vinci. Orange; very fine. F.

A 8 Maes. Splendid; scarlet; finest shaped flowers. Novelty.................. 100

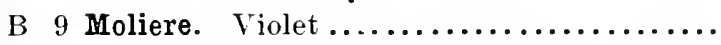

B 7 Mon Tresor. Yellow; extra fine. Best yel-

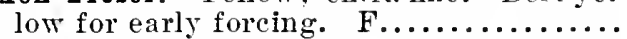

A 7 Nelly. Pure white; extra large flower.

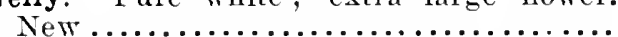

A 7 Ophir d'or. Golden yellow; extra large Hower. New exhibition sort ............ Pink Beauty. Bright pink and white. New

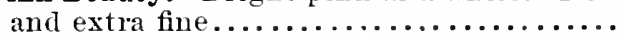

A 8 Pottebakker, Pure White. Best white. F..

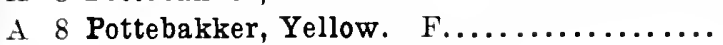

A 8 Pottebakker, Scarlet. Fine. F.........

B 8 President Lincoln (Queen of Violets)....... Prince de Ligny. Deep yellow; long stem.

B 9 Prince of Austria. True. Orange, shaded scarlet; very fine..................

B 6 Princess Marianne. Extra; wine, tinted pink; rery large. F................

Princess Wilhelmina. Fine deep pink and white; extra large flower..............

A 8 Proserpine. Queen of Tulips. Silky carmine-rose; perfect form. F...........

A 7 Purple Crown. Brownish crimson........

A 8 Rembrandt. Scarlet; fine..............

A 7 Rose Luisante. Superb, lirge; pink; extra

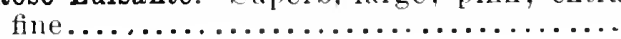

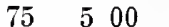

$20 \quad 125-950$

$25 \quad 150 \quad 1100$

$\begin{array}{lllll}45 & 2 & 75 & 21 & 00\end{array}$

$\begin{array}{lllll}30 & 2 & 00 & 15 & 75\end{array}$

$20 \quad 125 \quad 925$

$35 \quad 200 \quad 1500$

$\begin{array}{lllll}30 & 2 & 00 & 13 & 25\end{array}$

$\begin{array}{lllll}30 & 2 & 25 & 16 & 75\end{array}$

$40 \quad 250 \quad 20 \quad 00$

$40 \quad 250 \quad 2000$

$25 \quad 140 \quad 10 \quad 50$

$45 \quad 2 \quad 75 \quad 1950$

$\begin{array}{lllll}30 & 1 & 75 & 12 & 60\end{array}$

$25 \quad 150 \quad 10 \quad 50$

$\begin{array}{lllll}35 & 2 & 50 & 19 & 00\end{array}$

$60 \quad 400$

$\begin{array}{lllll}35 & 2 & 00 & 15 & 00\end{array}$

$40 \quad 275 \quad 2500$

$\begin{array}{lllll}30 & 1 & 75 & 12 & 70\end{array}$

$\begin{array}{lllll}30 & 2 & 00 & 15 & 00\end{array}$

$85 \quad 630$

$\begin{array}{lllll}35 & 250 & 19 & 00\end{array}$

$\begin{array}{lllll}35 & 2 & 00 & 16 & 00\end{array}$

$25 \quad 175$

1325

$70 \quad 500$

25150

$35 \simeq 00$

1100

1600

00700

$40 \quad 275$

$35 \quad 250$

1900

$35 \quad 175$

1300

$3 \overline{5} 185$

1300

$25 \quad 1500$

$\begin{array}{lll}30 & 200\end{array}$

35250

$40 \quad 285$

45350

$30 \quad 200$

$60 \quad 400$

1500

2200

2100

$1+00$

3000

$25 \quad 150 \quad 1150$

$75 \quad 450$

60425

$25 \quad 13 \ddot{0} 1000$

$50 \quad 350 \quad 2625$

$\begin{array}{lllll}85 & 5 & 50 & 35 & 00\end{array}$ 
SINGLE NAMED EARLY TULIPS, continued

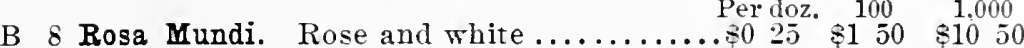

Rose Aplatis. Delicate pink; extra fine ... $50 \quad 350$

B 5 Rose Gris-de-lin. Beautiful pink; a splendid sort. F...................... $35 \quad 230 \quad 1800$

B 8 Rosy Queen. Deep pink, white-shaded. New...........................

B 8 Standard Silver. Crimson and white; a splendid rariets. F.................

A 8 Stanley. Pink reddish; fine for outdoors..

B 9 Thomas Moore. Buff, shaded orange. F. .

B 6 Van der Neer. Beautiful riolet...........

Van Goijen. Pink and white.............

B 8 Vermilion Brilliant. Dazzling scarlet: rers striking. F. .....................

C 8 White Swan. White; late, large and fine.

B 5 Wouverman. Splendid dark violet ........

B 7 Yellow Prince. Golden rellow ; sweetscented. Not good for outdoors. F.....

\section{$60 \quad 375 \quad 30 \quad 00$}

$40 \quad 2 \quad 75 \quad 2000$

$\begin{array}{lllll}60 & 4 & 00 & 27 & 00\end{array}$

$\begin{array}{lllll}30 & 2 & 00 & 15 & 75\end{array}$

$50 \quad 350$

$30 \quad 180$

$55 \quad+25 \quad 3200$

$\begin{array}{lllll}35 & 2 & 25 & 16 & 00\end{array}$

$50 \quad 350$

$30 \quad 175 \quad 1400$

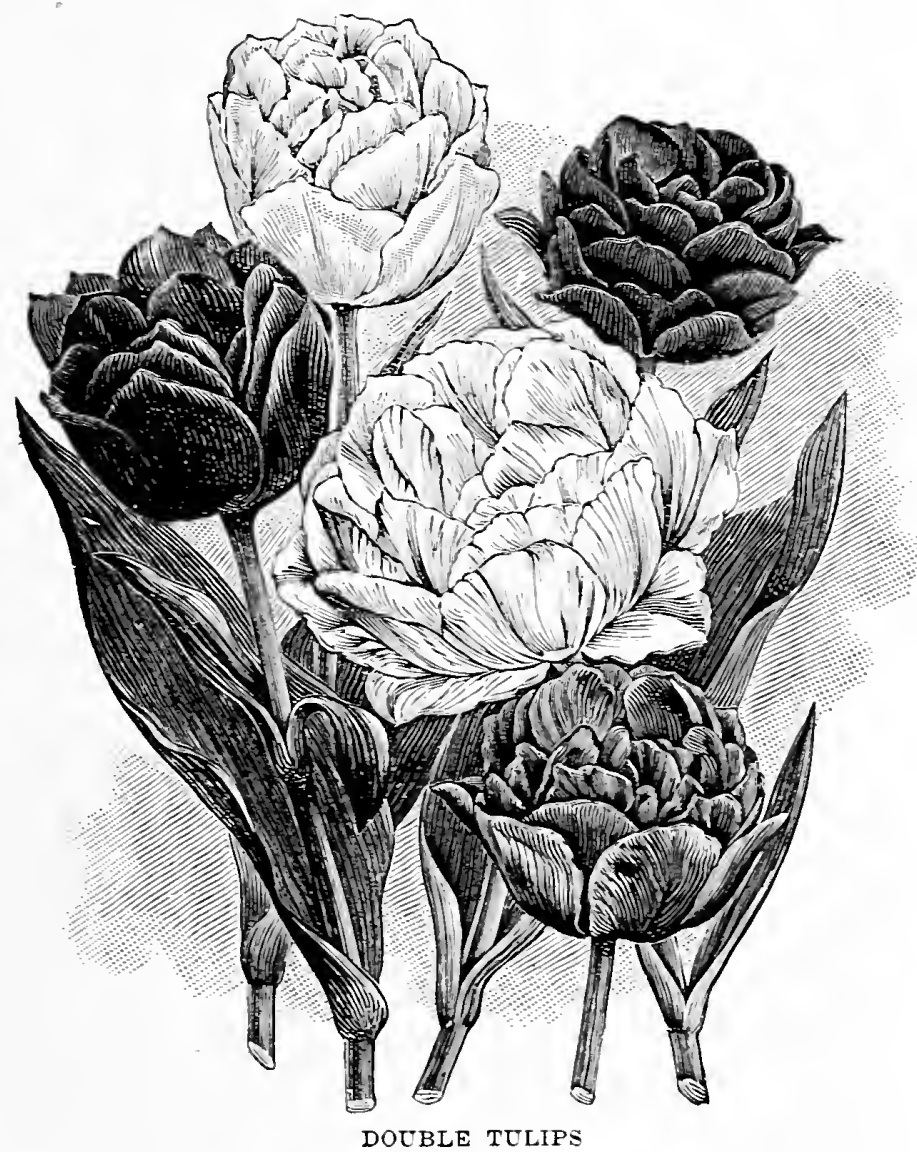

DOUBLE EARLY TULIPS

These are all rery desirable for bedding purposes. White, red and sellow sorts, of about the same height, planted in lines of three or four rows of each color, form, with their large rose-like flowers, beds of surpassing grandeur. Their flowers, being double, are quite persistent, and, being also late in blooming, ther serre to prolong the season of gorgeous beauts in the spring flower garden. Those sorts marked with an asterisk (*) are the earlier kinds, which are not onls valuable for bedders, but succeed admirably if forced in pots Those not so marked are not recommended for pot culture. A's flower together, B's follow, etc.

A 7 Alba Maxima. Pure white............ Per doz. $\begin{array}{llll}100 & 1,000 \\ 35 & 50 & \$ 19 & 00\end{array}$

A 9 *Couronne des Roses (Crown of Roses). Rich rosy pink: splendid ............. $85 \quad 625$

B $8 *$ Crown of Gold. Fellow............... i5 500

A 6 *Duc van Thol. Red and rellow: not desirable......................... $20 \quad 90 \quad 765$

B 8 *Duke of York. Purple, yellow edge ...... 30 I 85 12 75

Brimstone Beauty. Pinkish rellow: fine.. I $70 \quad 1200$
Cochenille. Scarlet ................ Per doz. $\$ 100$

1,000

A 7 Fulwellen Mantel (Velret Gem). Purple, brown and yellow................. $40 \quad 250$

A 7 *Gloria Solis Deep scarlet edge, with bright rellow......................

A 8 *Imperator Rubrorum. Brilliant scarlet;

fine for forcing, and one of the grandest
for bedding..........................
B 6 *La Candeur. Pure white; a splendid bed-

fine for forcing, and one of the grandest
for bedding.........................
B $6 *$ La Candeur. Pure white; a splendid bed-

$\begin{array}{lllll}30 & 2 & 00 & \$ 15 & 00 \\ 35 & 2 & 25 & & \end{array}$ ding kind. Used with Rex Rubrorum to form bedding designs, it makes a fine effect.............................

B 6 Murillo. Light pink; rery early; splendid for bedding and forcing............

A 8 Purple Crown. Purple-maroon ...........

A 7 Raphael. Delicate pink; extra fine; large flower ..................each, 16 cts... 1 i5 1000

B 7 Rex Rubrorum. Bright scarlet; splendid bedding sort, lasting a long time in perfection .......................... Rose Blanche. Pure white; rerr fine.... $25 \quad 160$ 12 25 Rose d'Amour. Extra fine; delicate pink; a beauty............... each, 10 ets... $100 \quad 625$

A 6 Rubra Maxima. Early; bright red..... $35 \quad 265$

A 7 *Salvator Rosa. Thite and deep rose.... $85 \quad 6 \quad 60$

A 7 *Tournesol, Red and Yellow ............ $35 \quad 250$

A 7 *Tournesol, Pure Yellow. Finest double yellow ...........................

A 7 Titian. Crimson-scarlet, edged with golden rellow; fine for bedding .............

Lucretia. Fine pink; large .............

Toreador. Orange-brown............. 13551000

\section{DOUBLE LATE TULIPS}

These bloom in May and are very shors and lasting.

Blue Flag. Tiolet-blue; distinct............ Per doz. $30 \quad \begin{gathered}100 \\ \$ 2\end{gathered} 00$

Buonaparte. Brown and riolet .............. 25 1 50

La Belle Alliance. Blue and white, feathered;

extra fine............................... $30 \quad 200 \quad \$ 1500$

Madame Catalina. Red and rellow............ $50 \quad 350$

Mariage de ma Fille. White, striped with

carmine ............................. t5 $300 \quad 23 \quad 25$

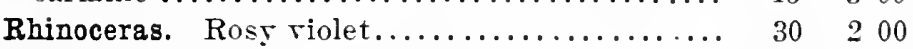

Yellow Rose. Golden sellow ............... $25 \quad 1750.25$

\section{PARROT TULIPS}

Parrot Tulips sometimes do not bloom satisfactorily, owing-to improper planting. Thes like a light, sand soil, shallow planting and a sunny location.

Parrot Tulips do not receire the attention they deserre. These belong to the late or Mar-flowering Tulips, and have inmense attractive flowers of singular and picturesque forms and brilliant and raried colors. The petals are curiously fringed or cut, and the form of the flower, especially before it opens, resembles the neck of a parrot. Thes form extraragantly showy flower beds and should be grown in every flower garden in quantities. The Parrot Tulips we import are from the most famous grower of these in Holland, and rery superior to those usually sent out, which are usually immature bulbs and will not bloom well under the most farorable circum. stances.

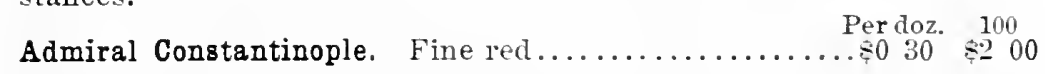

Cafe Brun. Brown and rellow striped ............... $30 \quad 200$

Cafe Pourpre. Orange, feathered witl scarlet ........... $60 \quad \pm 25$

Cramoisie Brillante, Scarlet: finest of all: free-blooming. $35 \quad 225$

Lutea Major. Yellow; rerr fine................... $30 \quad 200$

Markgraf von Baden. True. Orange, striped scarlet;

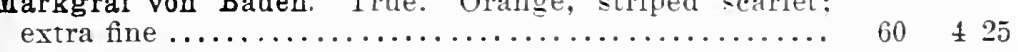

Perfecta. Fellow and scarlet.................. $35 \quad 225$

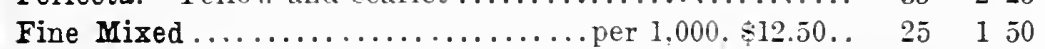




\section{MISCELLANEOUS TULIPS}

Late Tulips are but little known in this country, yet they are extremely beautiful and striking, and comeinto bloom in May, after all other Tulips are orer. Many are the original wild varieties, and, if planted where they can remain permanently, will improre year after year. Our experience has been that permanent plantations of Tulips should not be watered after flowering. They seem to do much better if allowed to take their chances with the weather, and we know of a lot of single early Tulips planted in a bed in a farm field where they have nerer been watered or cultirated in any way, not eren the weeds and grass remored, yet they thrive and bloom splendid!y year after year. We wish to call special attention to Gesneriana Tulip. Without exception, it is the most effective Tulip that can be planted outdoors. A bed of it will always attract the greatest almiration and enthusiasm.

Gesneriana. The tallest, largest flowers Per doz, $100 \quad 1,000$

showiest of all Tulips, for bed fing ond

masses. It deserres to be largely grown.

Height 18 to 24 inches. Flowers of enormous

size, on long, graceful stems, and the most

durable of all Tulips, as it holds its heautiful

color and keeps perfect for a long time, flow-

ering in May. Color rich crinsson-scarlet, with

glittering bine-black center. This is the Tulip

that attracted so much attention on the grounds

of James McKay, Esq., at Shadyside. Our

bulbs are guaranteed the true scarlet variety

and not the inferior purplish kind that is not

worth planting ....................... \$0 30 \$2 $00 \quad \$ 1600$

Golden Crown. Yellow, slightly edged with red: a splendid sort; similar to Gesneriana; fine

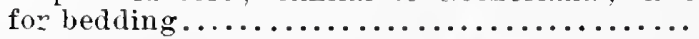

Late Double. Fine mixed; rery showy, large

$25 \quad 125 \quad 1000$

$20 \quad 125 \quad 1000$

\section{LATE SHOW TULIPS}

$\begin{array}{llll}\text { Bizarres Per doz. } 100 & 1,000\end{array}$ mixed varieties ..................... \$0 $30 \quad \$ 200 \quad \$ 1275$ 100 bulbs, in 25 named varieties, $\$ 3.50$.

Breeders. Self-colored; mixed............ $45 \quad 300$

Bybloems. Red on white ground. Choicest Doz. 100 mixed........................\$0 40 \$2 75 100 bulbs, in 25 named rarieties. $\$ 4$.

Roses. Red, on white ground. Choicest mixed rarieties.................... $40 \quad 275$

Violettes. Violet, on white ground. Choicest mixed $\ldots \ldots \ldots \ldots \ldots \ldots \ldots \ldots \ldots \ldots \ldots \ldots, 40 \quad 275$

The Bizarres, Bybloems, Roses and Violettes are the Tulips of the famous Tulip mania of Europe, when Tulips sold as high as $\$ 1,000$ each, and entire communities were involved in the speculation. They are very interesting and beantiful.

\section{MAY-FLOWERING TULIPS}

Blushing Bride (Shandon Bellor doz. $100 \quad 1,000$

Beautiful rose-pink,

shaded white; fine form A most

superb and distinct variety ....\$0 $50 \quad \$ 3 \quad 50 \quad \$ 26 \quad 00$

Bouton d'0r (Yellow Gesneriana).

Deep golden rellow; of great

value for outdoor cutting; splen-

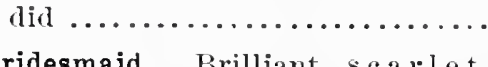

striped pure white; distinct and rery beautiful .................

Clusiana. The Larly Tulip. Red.. 125

Darwin. A new race of Tulips of remarkable beauty and brilliancy of coloring. Fine

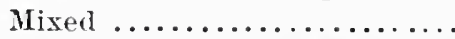
100 bulbs, in 25 named varieties, $\$ 4.50$.

Elegans. A grand Tulip. Very slowy, rich crimson-scarlet; large flower.............. $175 \quad 1200$
Florentine. Sweet-scented; yellow...........\$0 Per doz. $45{ }^{100} 50$

Greigi. Odd and beautiful........ each, $15 \mathrm{c} . . .150 \quad 1025$ Haageri. Red, with black ceuter.......... 85

Kauimanniana. White; large; early.each, $35 \mathrm{c} . . .3502500$ La Merveille. Orange-red; large............ $150 \quad 1000$ Maculata. Carmine, black center .......... $60 \quad 325$ Mrs. Moon. Pure yellow; large.....each, 75c... 800

0culus Solis. Norel, beantiful rariety; dazzling scarlet.......................... 1 00750

0strowskiana. New. Scarlet ............. 125800

Parisian Yellow. Golden yellow, painted flow-

ers ............................. $65 \quad 425$

Persica. Fragrant yellow.............. 150

Picotee, or Maiden's Blush. An elegant, longshaped, clear white flower; the petals, which are pointed and elegantly refiexed, are beautifully margined and penciled on edges with bright pink....................... $40 \quad 2 \quad 60 \quad \$ 20 \quad 00$

Retroflexa. Clear yellow................ 125900

Viridiflora. Green ................... $150 \quad 900$

Vitellina. Sulphur-yellow; large.......... $175 \quad 1200$

Con

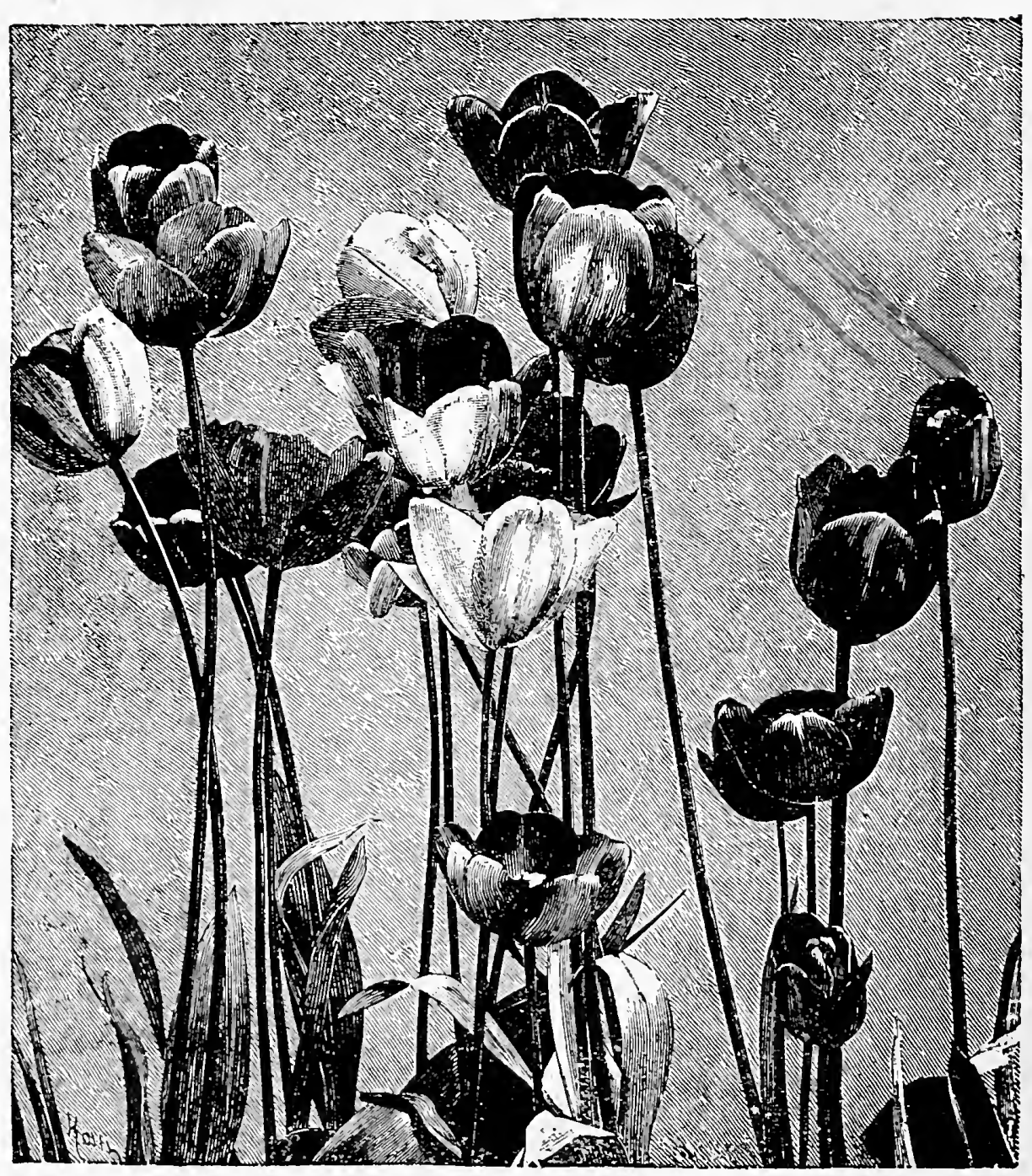

LATE SHOW TULIPS. From the English Flower Garden 


\section{-}

3.tan

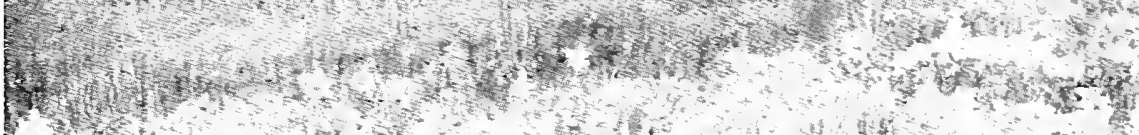

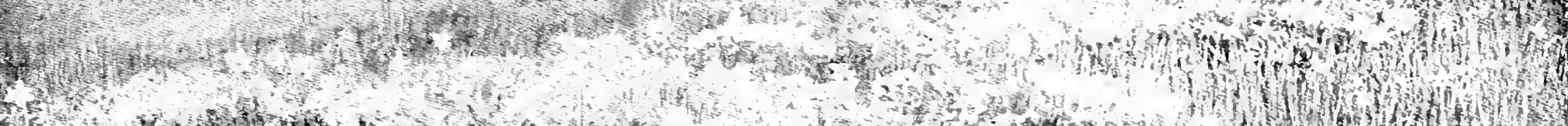

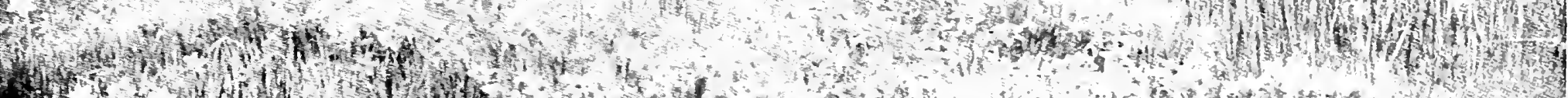

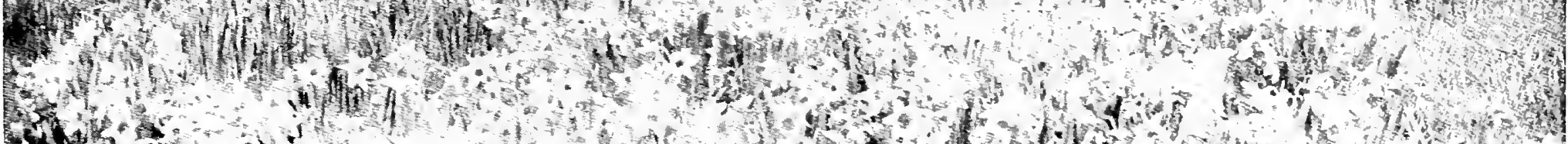

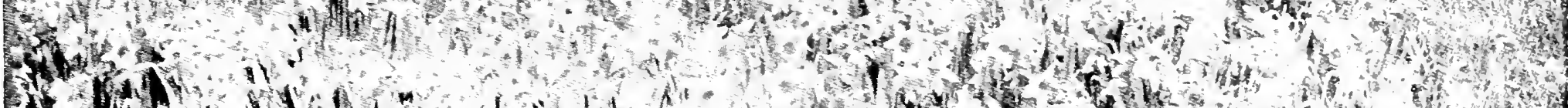

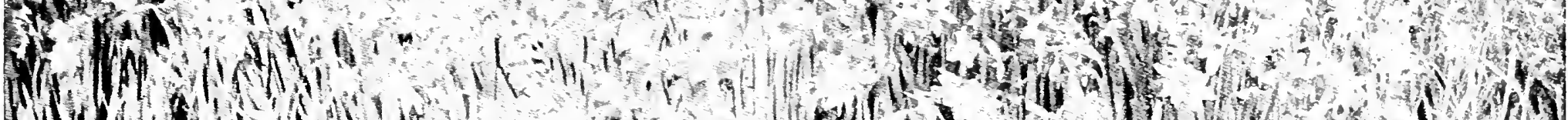

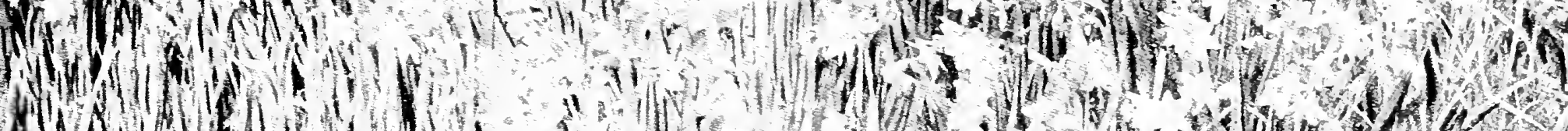

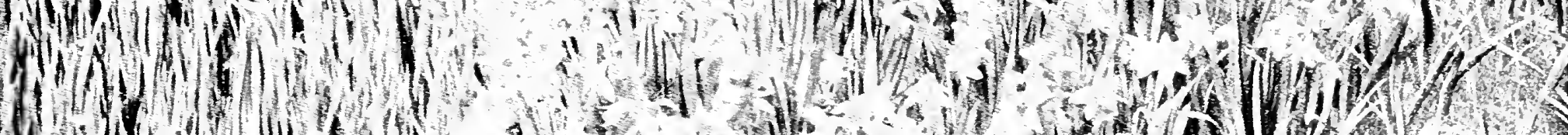

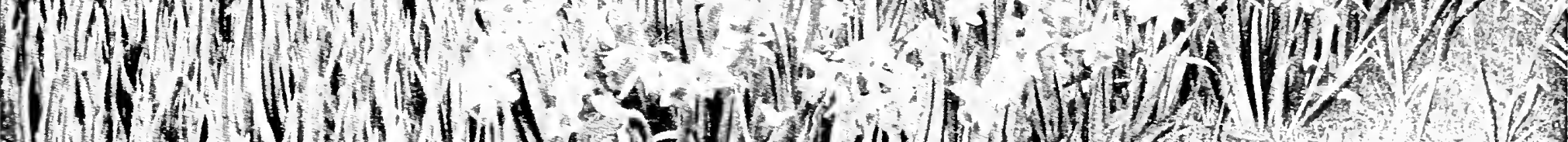

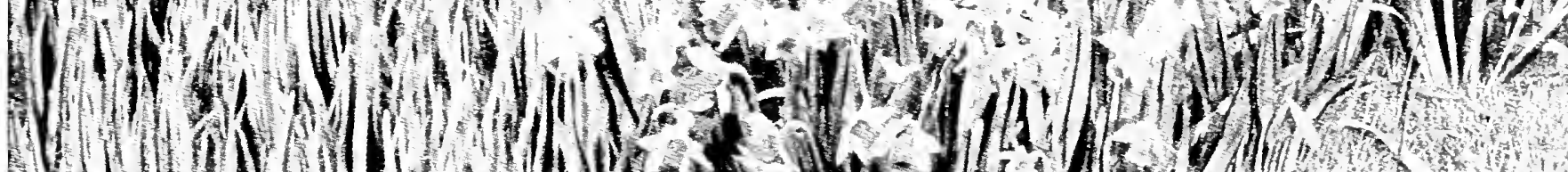

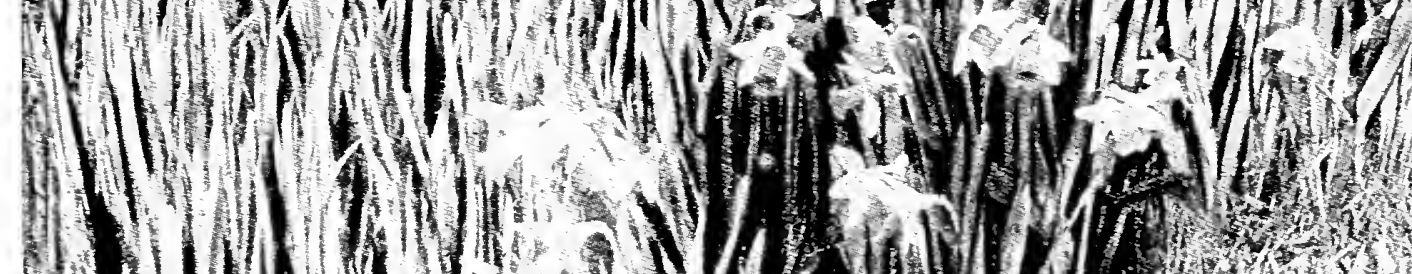
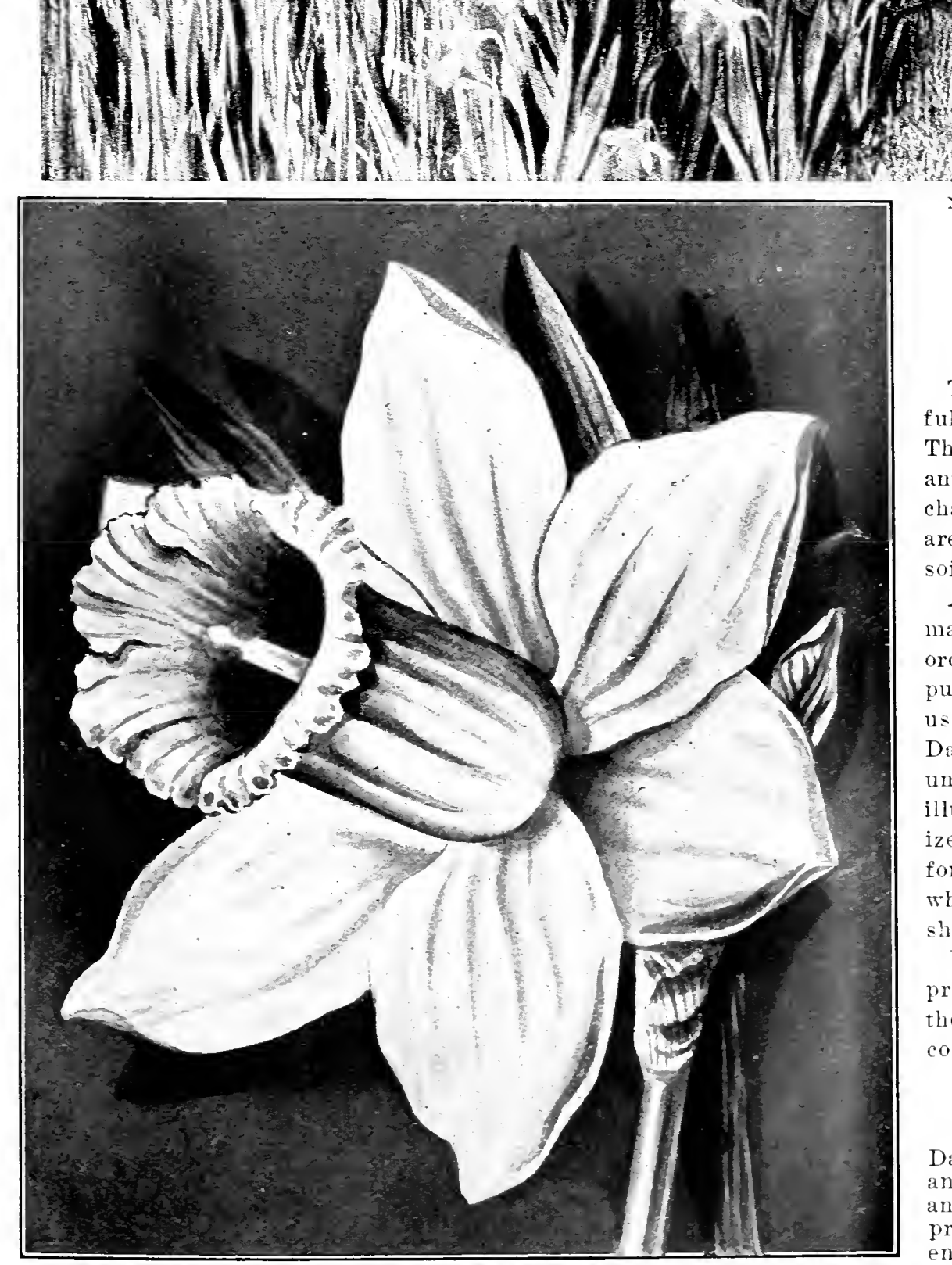

Narcissus

The Narcissi, or Daffodils, are undoubtedly the most beautiful, interesting and useful of all spring-flowering bulbs. The flowers hare a very great diversity of form. more so than any other family, and they are exquisitely graceful and charming. One of their most desirable qualities is that they are a permanent addition to the garden. and. planted in good soil, the increase in size and beauty year after rear.

A majority of the rarieties are of the easiest culture, and many can be naturalized in the grass. in open woods or purpose many rarieties are so inexpensire that the can be used by the thousand. It must alwas he remembered. when Daffodil are planted in the grass. that it must not be cut until the foliage of the Daffodils ripen-. about Juls 1. The illustration shows how beautiful Daffodils are when naturalized. The Daffodils are all fine for cut-flowers. either for forcing or for growing outdoors: and. at the low prices at which mant of them can be had, there is no reason why they should not be grown by the hundred and thousand.

We take great pleasure in making a substantial reduction in price for some of the finest rarieties. also in offering mant of the rarer kinds from England not before obtainable in this country.

\section{Narcissus Emperor}

This is the largest and finest deep sellow trumpet-flowered Dafforlil in cultiration, and is realls superb. both in foliage and flower. A few years ago it was selling at $\$ 25$ per 100 , price. per 100. but a contract made in Holland this sear enables me to offer fine bulbs at the following prices: 60 cts. NARCIEST'S EMPEROR per doz.. \$3.s0 per 100. $\$ 34$ per 1.000 . 


\section{SPECIAL OFFER OF GOLDEN SPUR NARCISSUS}

(See illustration, page 21)

This is one of the most valuable of the Trumpet Narcissi, with magnificent deep yellow trumpet with brim expanding like Maximus, and large, spreading, full deep yellow petals. It is free-flowering and splendid both for house culture and outdoors, and is one of the most desirable varieties for naturalizing. By contracting for one hundred and fifty thousand bulbs of this variety, we are enabled to offer them at the following prices, which are less than half the prices usually asked:

Per doz. $100 \quad 1,000$

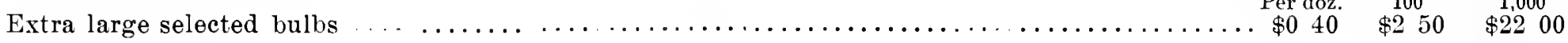

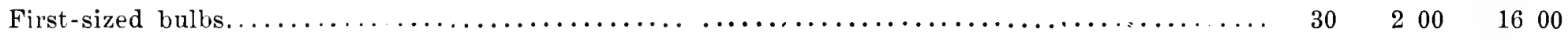

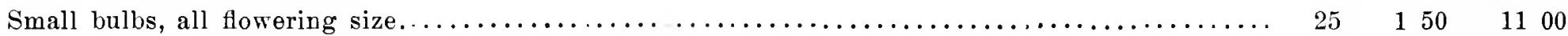

\section{SUPERB NEW NARCISSI EXTRAVAGANZA HYBRIDS}

These superb new Narcissi are now offered for the first time, and can not be had elsewhere either here or in Europe, as the originator, one of the most famous bulb-growers inoHolland, has given us the control of the entire stock. He writes us as follows: "These hybrids have been raised from a cross between selected stock of Poeticus ornatus and the beautiful Princess

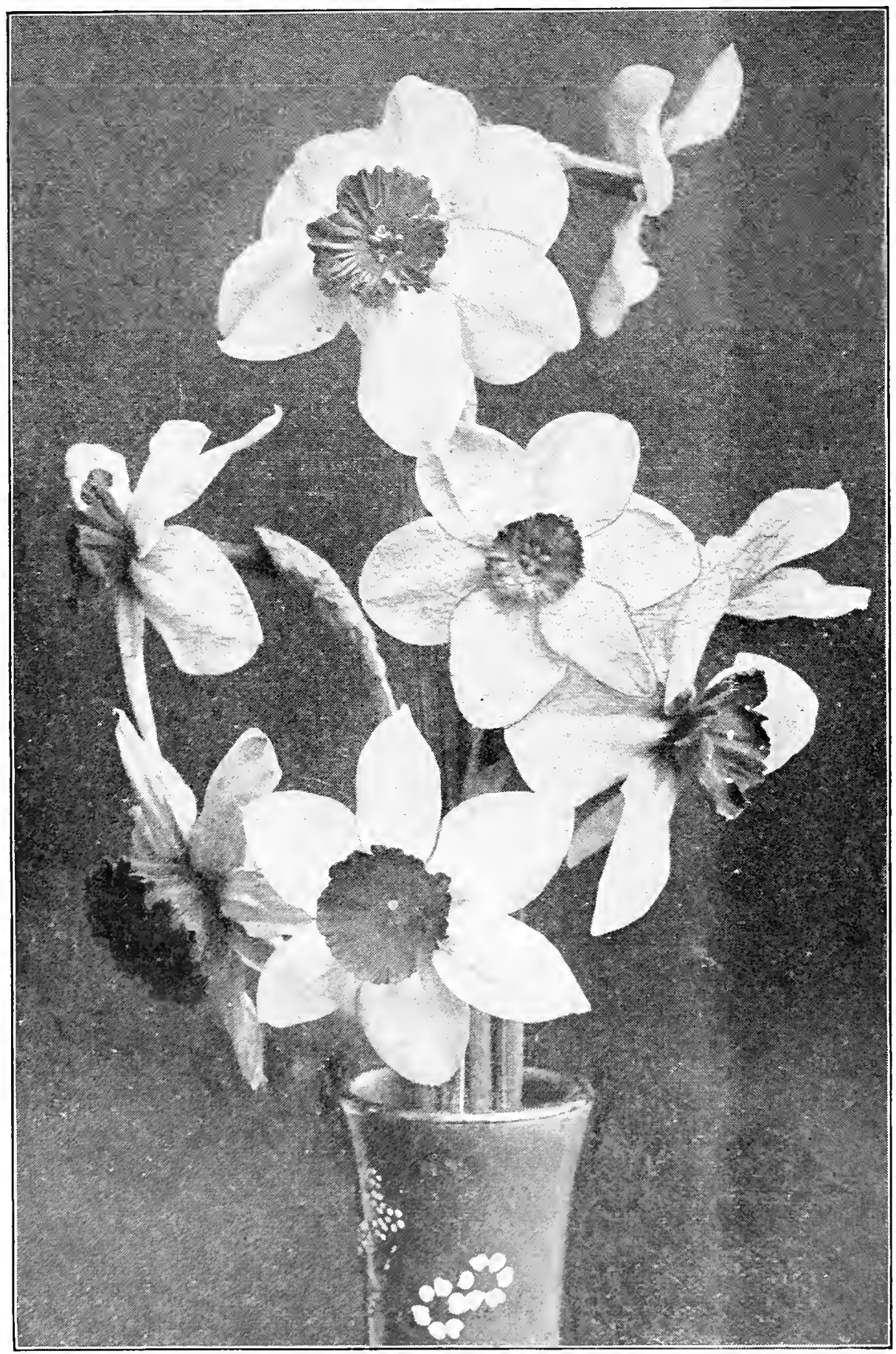

NARCISS1, EXTRAVAGANZA HYBKIDE
Mary of Cambridge, thus combining the freeblooming habit of Ornatus with the perfect form of Princess Mary, and nearly all have the orangestained cups, thus making them equal to the exorbitantly high-priced new English varieties, and they are the most charming flowers for cutting owing to the great variety of flowers among these seedlings. Knowing that your house handles only the highest grade bulbs and commands the finest retail trade in America, we have selected it for the introduction of this valuable novelty, and have reserved our entire stock for your use."

85 cts. per doz., $\$ 6$ per 100, $\$ 85$ per 1,000

\section{OXALIS}

These are profuse bloomers, and very attractive in the house or conservatory during the winter. Plant three or four bulbs in a pot; the pot should be kept near the glass to prevent the foliage from growing too long.

Bermuda Buttercup. Greatly improved selection from Oxalis Cernua; grows in the congenial soil and climate of Bermuda until the bulbs have attained great strength, producing plants and flowers larger and more luxuriant than the common variety. It is one of the finest of winterflowering plants for pot culture, of strong, luxuriant growth; one bulb is sufficient for a 6 -inch pot. The great profusion of bloom produced in uninterrupted abundance for weeks is astonishing. The flowers are of the purest bright buttercup yellow.

Bowiei. Brilliant rose.

Hirta. Rose-colored.

White (Multiflora alba).

Price of any of the above, 3 cts. each, 25 cts. per doz., $\$ 1.50$ per 100

Mixed, containing a large number of varieties. 5 cts. for 3,15 cts. per doz., $\$ 1$ per 100 . 


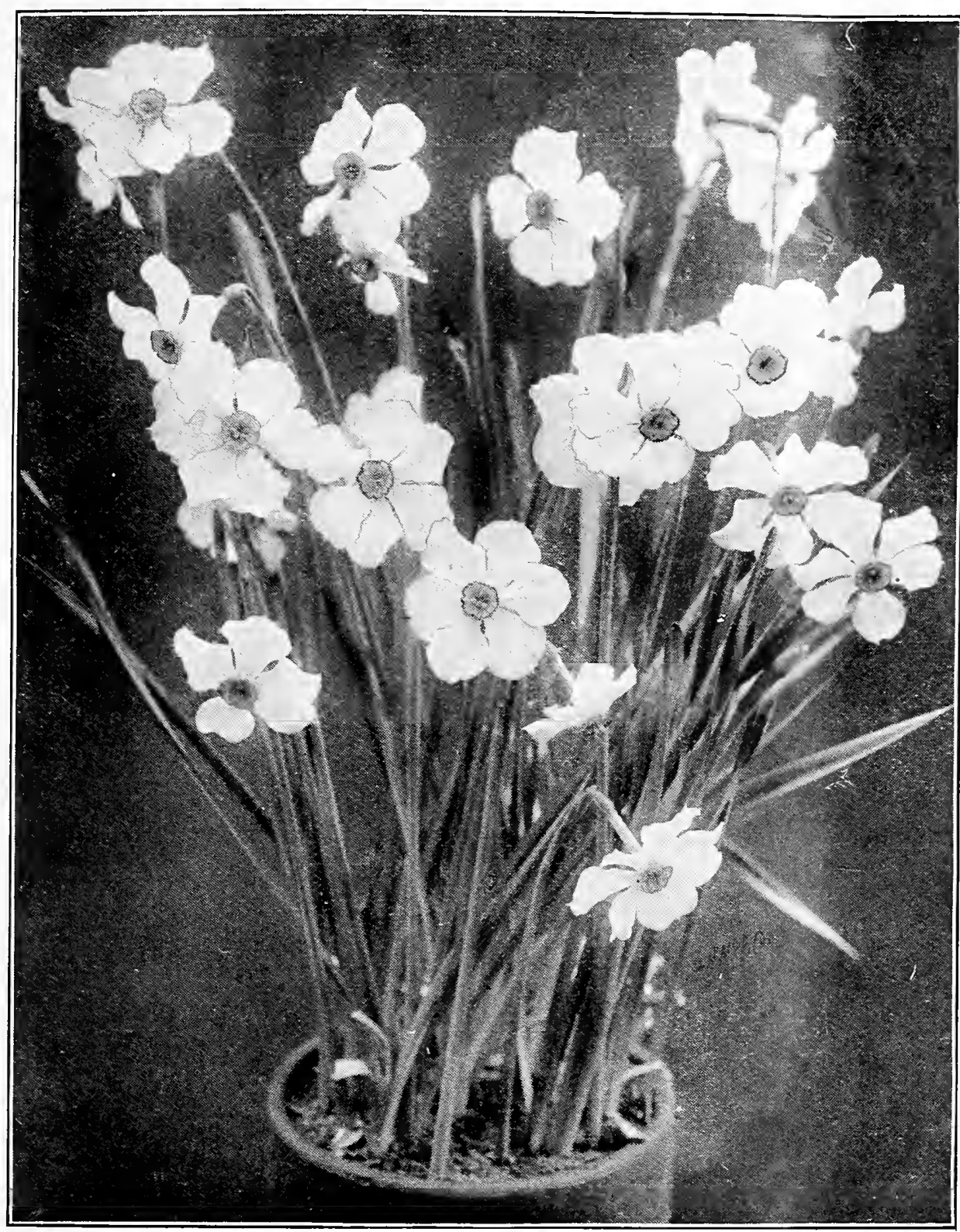

NARCISSUS POETICUS ORNATUS (Bloomed in the house)

\section{Narcissus, Bicolor Empress}

This may be considered rather a high-priced Daffodil for planting in the grass, but the effect produced is so lovely and it is so hardy and vigorous and increases so rapidly that nothing can be planted that will be more satisfactory, and, as we control the best stock of it in Holland, we can make a specially low price in spite of the 25 per cent duty now levied on bulbs. If planted in orchards, meadows or any place where the grass is not mown by a lawn-mower, these Daffodils will increase in quantity and beauty year after year. Where they are planted the grass must not be mown before the middle of June, so that the bulbs may have time to ripen before the tops are cut off. Price, $\$ 385$ per $100, \$ 34.50$ per 1,000 . See illus tration on opposite page.

\section{DOUBLE NARCISSUS}

$\begin{array}{llll}\text { Per doz. } & 100 & 1,000\end{array}$ Alba plena odorata. Double Poeticus. Pure white, resembling a Gardenia, sweet-scented: fine for outdoors in a dry soil.................... $\$ 0 \begin{array}{llllll}18 & \$ 1 & 10 & \$ 9 & 50\end{array}$

Incomparabilis fl, pl. Butter and Eggs. Full double flowers of rich yellow................

Jonquils. Double; sweet-scented..............

Orange Phœnix. Eggrs and Bacon. Double; white and orange; beautiful. ..................

Sulphur, or Silver Phœnix. Pure white, beautiful flower; splendid........................

$20 \quad 125 \quad 1000$ $\begin{array}{lllll}35 & 2 & 10 & 18 & 00\end{array}$

$\begin{array}{lllll}30 & 1 & 75 & 13 & 00\end{array}$ $65 \quad 350 \quad 3000$
Van Sion. Double Yellow Daffodil. Per doz. 100 1,000 One of the best forcing sorts; extra large fine bulbs, and not mixed with the inferior greenish flowered

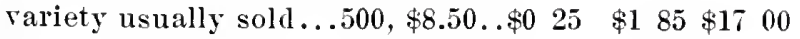

\section{SINGLE NARCISSUS}

The true Poet's Daffodils. Delicately perfumed and charming in form and color. Fine for planting among shrubbery or in the grass.

Per doz. $100 \quad 1,000$

Jonquils. Single; sweet-scented...\$0 $\begin{array}{llllll} & 15 & \$ 0 & 85 & \$ 6 & 50\end{array}$ Jonquils (Campernelles). Yellow ; sweet-scented..................

Jonquil (Campernelle) rugulosus. Large golden yellow: larger and finer than the old Campernelles..

Poeticus. Pheasant's Eye. The wellknown Poet's Narcissus. 500, $\$ 2.25$.

Poeticus Burbidgei. Pure white cup, margined with cinnabar-red; early.

Poeticus ornatus. Improved Poeticus. Can be forced earlier and blooms earlier outdoors.................

Poeticus poetarum. Pure white petals, with orange-scarlet cup... $\quad 60 \quad 375$

Poeticus grandiflorus. Large flowers. $150 \quad 1100$

\section{TRUMPET NARCISSUS}

Per doz, $100 \quad 1,000$ perianth .................. \$0 65 \$3 50 \$26 00

Barri conspicuus, Broad primrose petals and elegantly expanded crown, heavily stained orangescarlet; extra fine............

Bicolor Empress. Pure white perianth, with yellow trumpet; extra large, superb flower. This variety is an improved Bicolor Horsfieldi, and

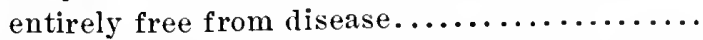

Bicolor Horsfieldi. King of Daffodils. Very large flowers, with pure white perianth and rich yellow trumpet; strikingly beautiful; fine for outdoors or for forcing. A popular sort, but decays badly in the packages. We will import it at purchaser's risk only. We advise order-

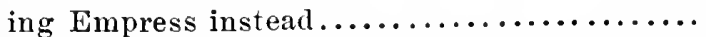

Bicolor grandis. White perianth, with yellow trumpet; more dwarf and a little later than

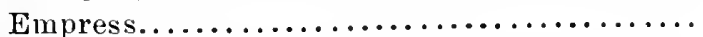

Bicolor, Mrs. Walter T. Ware. Perianth pure white, trumpet yellow; new and fine.........

Bicolor Victoria. A novelty of striking beauty.

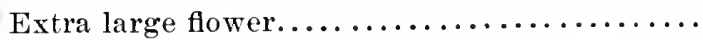

Bulbocodium. Hoop Petticoat Narcissus. Rich golden yellow; fine and curious.............

Emperor. The largest Daffodil in cultivation; golden yellow; superb. This Daffodil should be in every collection; it is really grand......

Glory of Leiden. Perianth and trumpet deep yellow. The largest and finest of the newer Daffodils. A superb and distinct variety that should be included in every amateur's collection of choice Daffodils................2 $40 \quad 1700$
$60 \quad 3 \quad 85 \quad 3450$

60400 
TRUMPET NARCISSUS, continued

Golden spur. Large, deep yellow trumpet, with Perdoz. $\quad 100 \quad 1,000$ brim expanding like Maxinus, and large, spreading, full, deep yellow petals. This is one of the very largest anil most beautiful Daffodils in eultivation, and should be used freely, both for outdoor culture and for forcing........... \&n $40 \quad \$ 2 \quad 50 \quad \$ 2200$

Henry Irving. Bold golden yellow trumpet, with vellow wheel-shaped perianth; very fine. 'The flowers of this fine variety stood 13 degrees of freezing in my garden this spring without

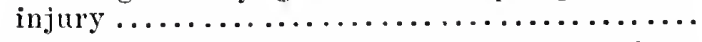

Incomparabilis Figaro. Perianth and cup yellow, stained orange; very large and spreading; fine.

Incomparabilis simplex. Yellow; fine.........

Incomparabilis alba stella. Large white perianth, with a yellow cup; a most charming and lovely variety, either for outloors or forciug........

Incomparabilis Cynosure. Large sulphur-white

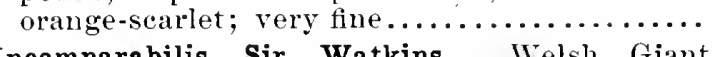

Incomparabilis Sir Watkins. Welsh Giant Daffodil. Very large petals of a rich sulphuryellow color, and large cup, yellow, tinged with orange; the immense flowers are sometimes $5 \frac{1}{2}$ petals, cup also sulphur-white, stained witl

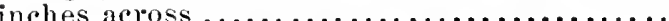

Mrs. Langtry. Pure white; twin-flowered; a gen for forcing and for cutting................ \$0 $40 \quad \$ 2 \quad 00 \quad \$ 1600$ Mad. de Graaf. The largest and finest of the white Trumpet Daffodils.........Each, $\$ 1.25$. .

Major. Trumpet Major. Golden yellow petals and trumpet; very free bloomew; early. This variety may be forced easily and early. Also

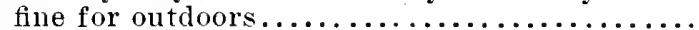

Maximus. Hale's Beaten Gold. Deep golden yellow perianth and trumpet.............. $\begin{array}{lllll}45 & 2 & 75 & 23 & 00\end{array}$

$20 \quad 125 \quad 900$

$20 \quad 125 \quad 900$

$20 \quad 1 \quad 10 \quad 950$

$25 \quad 125 \quad 1000$
Obvallaris. Tenby Daffoclil. Deep yellow; very

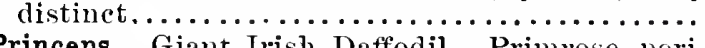
Princeps. Giant Irish Daffodil. Primrose rerianth, with yellow trumpet; extra file ........

Triandus albus. Angel's Tears; Cyclamen Daf-

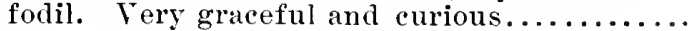

$25 \quad 150 \quad 1300$

$85 \quad 550$

$25 \quad 125 \quad 950$

85600 $\begin{array}{rrrrr}70 & 5 & 00 & 36 & 00\end{array}$
NARGissus ORANGe PHGenix (See page 19)

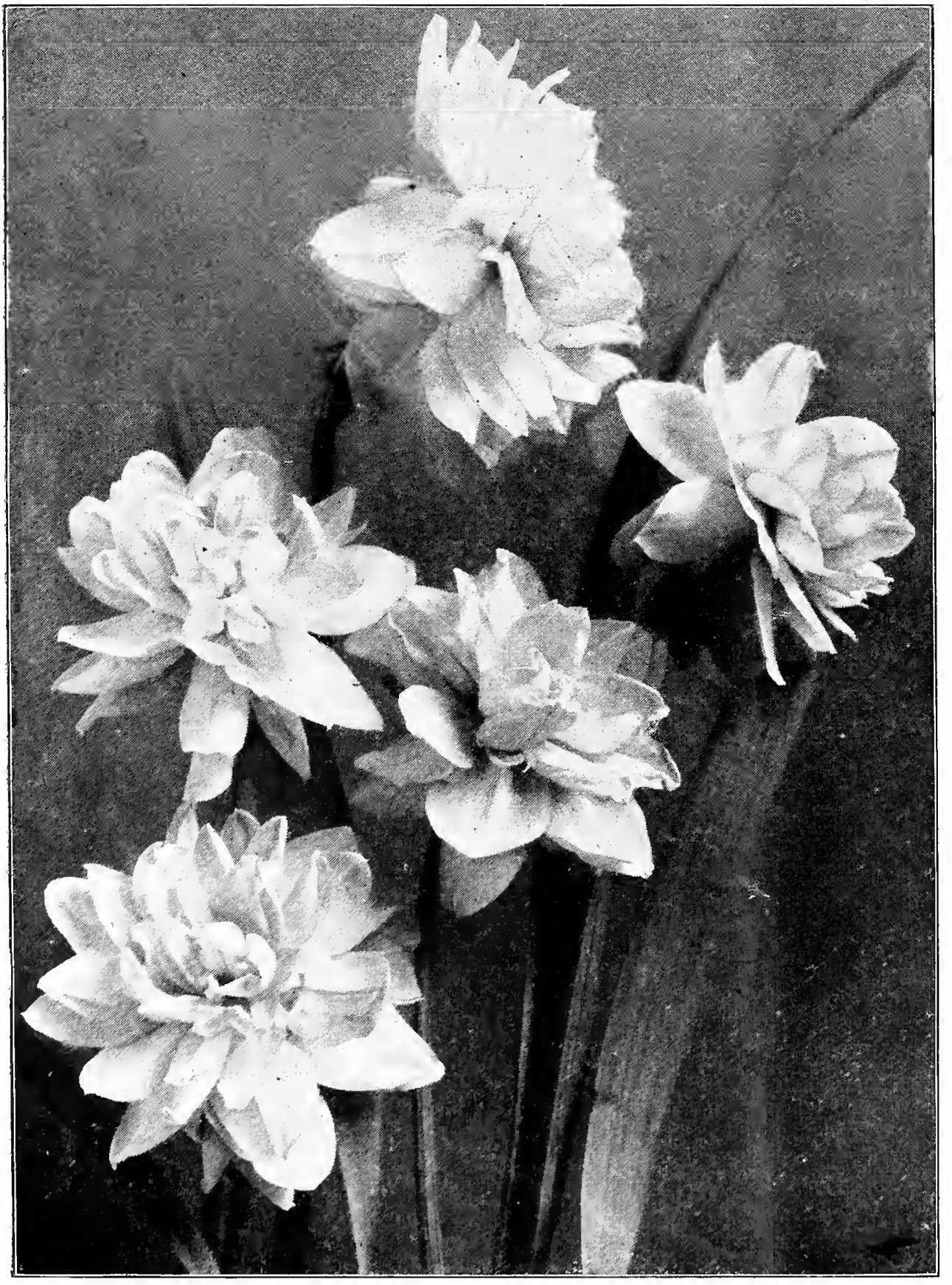

\section{POLYANTHUS NARCISSUS}

These Narcissi produce fine large clusters of flowers, which bave a very delicious scent. They may be forced very easily and early, and are also fine for outdoors.

Notw.-Polyanthus Narcissi, although not considered hardy, luave always proved hardy with us with a covering, and produce much larger flowers and more of them than those grown in the house in pots.
100 bulbs, in 10 fine named sorts, our selection, for $\$ 4$

Chinese Sacred. The much-advertised Per doz. 100 "Chinese Sacred Lily"; of little value.\$1 $15 \quad \$ 700$

Fine Mixed, without names......... 40 250

Gloria Mundi. White, with citron cup;

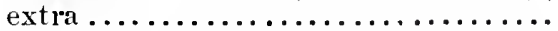

Gloriosa. Pure white, with orange cup; very fine; extra; distiuct...........

Grand Monarque (true). Large; pure white, with citron cup; extra fine. This variety is one of the finest of all white Polyauthus Narcissi ..........

Grand Soleil d'Or. Yellow and orange;

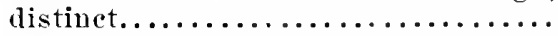

Her Majesty. Dwarf; white, with yellow cup; finest and largest variety of

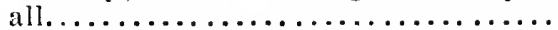

Lord Canning. Dark yellow; extra fine. Newton. Very fine yellow, with orange cup; distinct; extra free bloomer...

Paper White. Fine for foreing; can be had in bloom early in December; bulbs ready in August............. per $1,000, \$ 10$.

Paper White. New; large-flowered.... per $1,000, \$ 13$.

The Pearl (true). Pure white, large flower..................... 75, 450

\section{COLCHICUM}

\section{(Autumn-Flowering Crocus)}

These charming flowers resemble crocuses, but are larger and are displayed more above the ground, and, instead of blooming early in spring, bloom in September, when the flowers appear without any leaves, the leaves appearing the following spring. The bulbs are very apt to bloom in the packages while being delivered, but this will not prevent them from blooming the following season. The colors comprise many shades of white, purple, rose and striped. They are beautiful and interesting and should be planted where they can remain permanently.

Autumnale alba. White............ Per doz. $\$ 0 \quad 85 \quad \$ 60$ Autumnale major. Purple........... $150 \quad 1000$ Autumnale purpurea plena. Double purple..................... $150 \quad 1000$ 

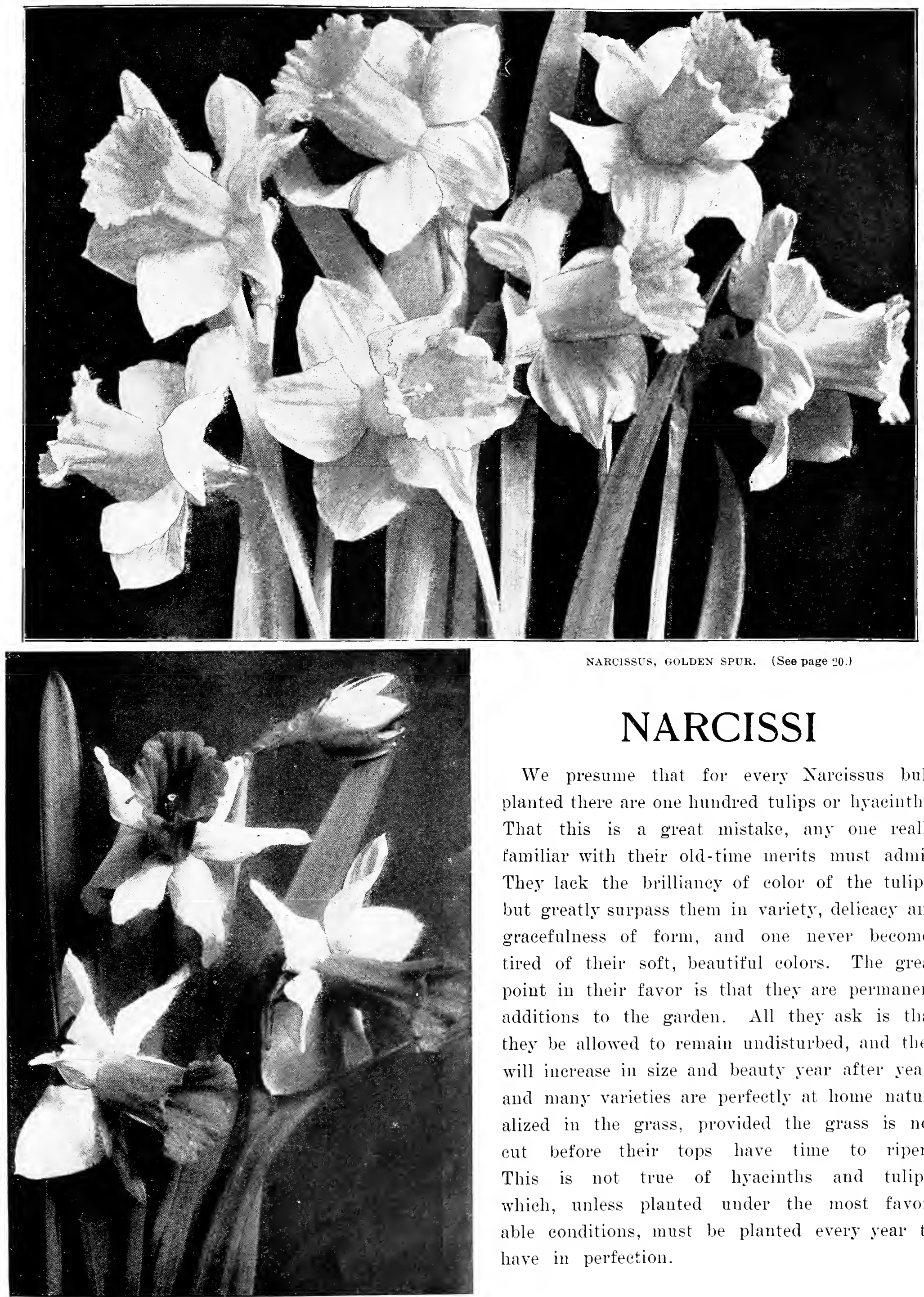

NARCiSsus, GOLden SPUR. (See page 20.)

\section{NARCISSI}

We presume that for every Narcissus bulb planted there are one hundred tulips or hyacinths. That this is a great mistake, any one really Pamiliar with their old-time merits must admit. They lack the brilliancy of color of the tulips, but greatly surpass them in variety, delicacy and gracefulness of form, and one never becomes tired of their soft, beautiful colors. The great point in their favor is that they are permanent additions to the garden. All they ask is that they be allowed to remain undisturbed, and they will increase in size and beauty sear after sear, and many varieties are perfectly at home naturalized in the grass, provided the grass is not cut before their tops have time to ripen. This is not true of hracinths and tulips, which, unless planted under the most favorable conditions, must be planted every year to have in perfection. 


\section{Miscellaneous Bulbs and Plants}

Prices for orders received before July I, 1905, for fall delivery

Prices on any bulbs not offered in this Cataiogue will be furnished on application

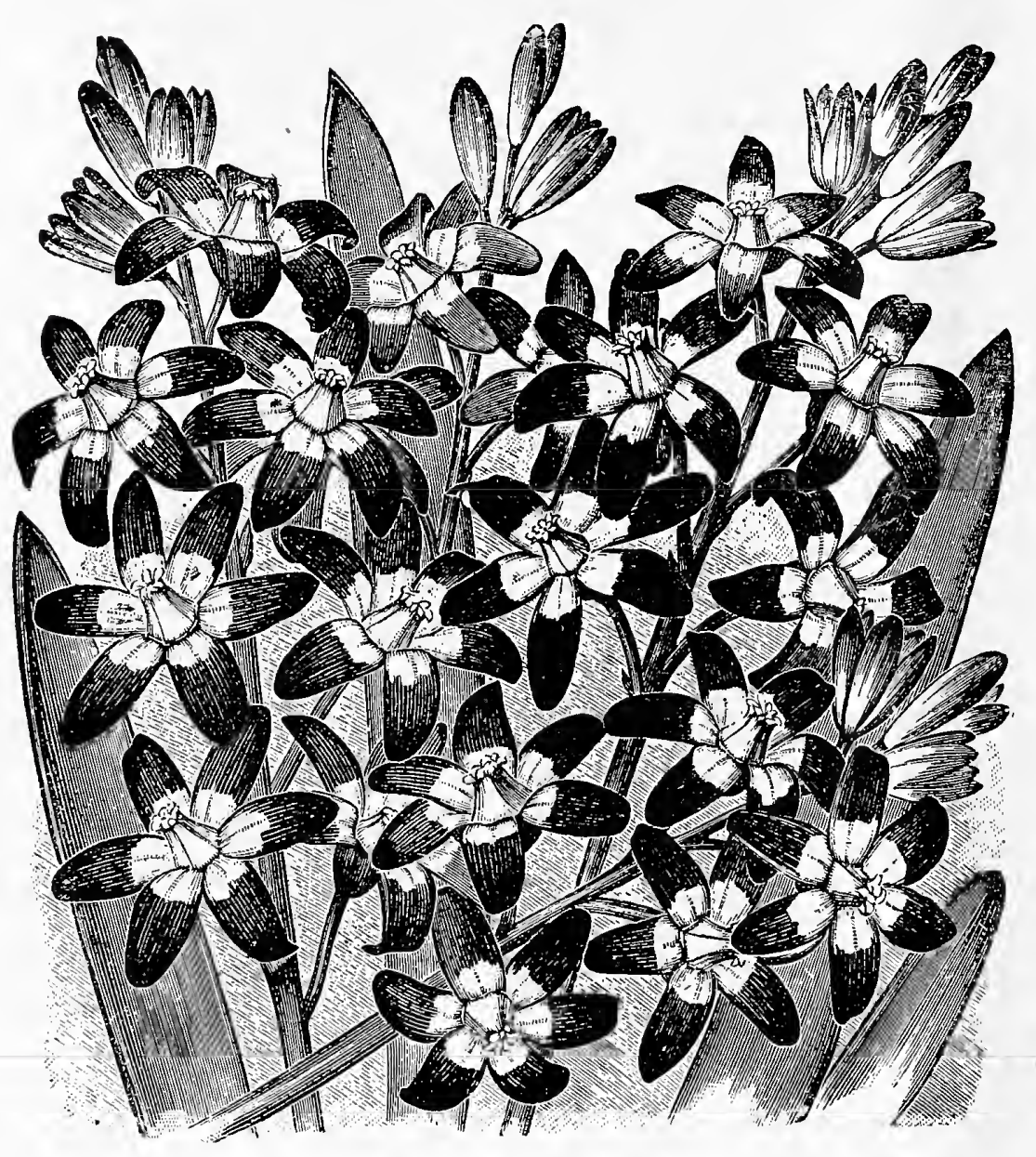

CHIONODOXA LUCILIE

ALLIU Per doz. 100

Lermettil grandiflorum. Pinkish white..\$0 $20 \quad \$ 125$

Moly Luteum. Yellow....................... 15 . 75 \$ô 00

Neapolitanum. Pure white; fine................. $18 \quad 125 \quad 700$

ALSTREMERIA Chinensis. Ready in November. Seedlings, mixed...........................

ANEMONE coronaria, Double, Large Scarlet.......

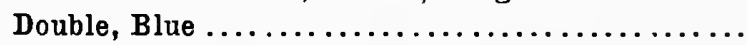

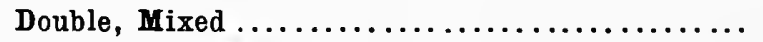

100 bulbs in 90 fine named varieties ............

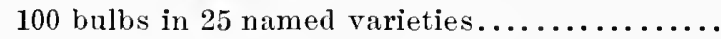

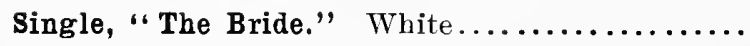

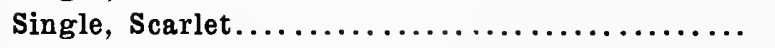

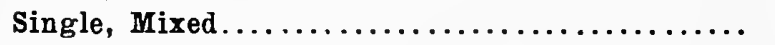

ChIONODOXa Alleni. New. Very large..........

Gigantea. New. A great acquisition, differing from all others of this family by its unusually large flowers of lovely lilac-blue, with conspicuous white center. Is thoroughly hardy and a perfect gem for spring decoration in masses in the garden, and when grown in pots for winterflowering it is beautifully effective .............

Luciliæ (Glory of the Snow). Very lovely; fine

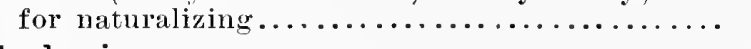

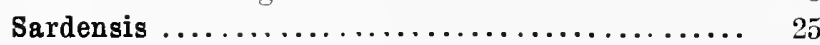

CROWN IMPERIAL, Aurora. Rel............. 250

Lutea. Yellow........................... 225

Rubra folia aurea variegata Golden variegated

foliage ................................. 200

Rubra maxima........................ 450

Fine Mixed ............................. 140

$$
160 \quad 1200
$$$$
160 \quad 1200
$$

ERYTHRONIUM Americanum doz. 100 1,000 Violet). Extremely beautiful and fine for naturalizing...................... $\$ 100 \quad \$ 700$

Large-flowering, Mixed .............. $30 \quad 2 \quad 00 \quad \$ 16 \quad 00$

FRITILLARIA aurea. New. Yellow, spotted; extra fine .........................

Meleagris. Fine mixed seedlings .........

GALANTHUS Elwesii (New Giant Snow-

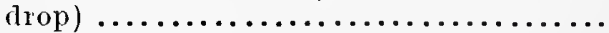

Nivalis (Single Snowdrop)..............

Nivalis (Double Snowdrop) ...............

GLADIOLUS Colvillei alba, "The Bride." True. Very popular in England for

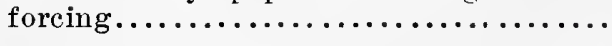

IRIS, Anglica (English Iris). 100 roots in 20 named varieties .................. Anglica, Mont Blanc Pure white; large and fine. This is the variety grown so largely in England for cut-flowers.......

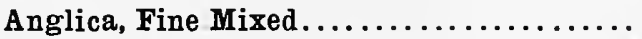
Germanica (German Iris). Americangrown. In 25 named varieties......... 100 Germanica, "Silver King." A most beautiful variety; of silvery white color....... 125

Hispanica (Spanish Iris). 100 roots in 20 named varieties .......................

Hispanica Blanche Fleur. Pure white. The best white Spanish Iris............ Hispanica, Chrysolora. The best yellow, and the variety so largely grown for Covent Garden Market in London.........

Hispaniea, Count of Nassan. The firest dark blue .........................

Hispanica, Louise. Beautiful shade of light blue .........................

Hispanica, Fine $\mathbb{Y}$ ixed ................ 15

Iberica (Rhizomatous). Very fine........ 150

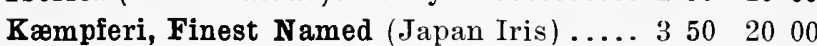
Kæmpferi, Mixed varieties ............. $125 \quad 6$ C0

Lortetti. Pale creamy pink, with brown spots; extra fine............each, $\$ 1.25 . .1200$

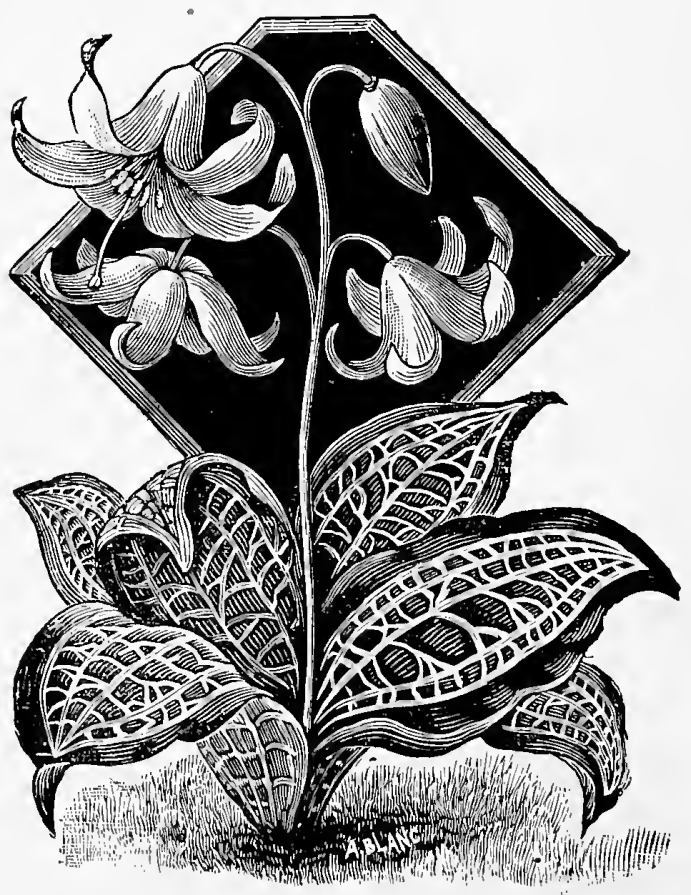

ERYTHRONIUM AMERICANUM
$85 \quad 500$

$40 \quad 2 \quad 40$

$\begin{array}{lllll}20 & 1 & 00 & 8 & 00\end{array}$

$\begin{array}{lllll}15 & 1 & 00 & 8 & 25\end{array}$

$\begin{array}{lllll}30 & 2 & 00 & 12 & 50\end{array}$

$\begin{array}{lllll}25 & 1 & 70 & 10 & 00\end{array}$

425

325

2400

$\begin{array}{llll}2 & 25 & 17 & 00\end{array}$

500

800

200 


\section{MISCELLANEOUS BULBS AND PLANTS, continued}

IRIS Pavonia (Peacock Iris). Pure white, with blue doz. 100 blotch.................................\$0 30 \$1 50

Persica. Sky-blue, with yellow streaks; blooms very

early in spring............................ 2 $00 \quad 1200$

Reticulata. Deep violet, blotehed with yellow........ $\cong 25 \quad 1900$

Rosenbachiana. Dwarf; splendid grayish blue, with yellow spot on the lower petals of the flowers blooms in Jareh..................each, $\$ 1.50$.

Sibirica. American-grown. White and blue....... $75 \quad 500$ susiana (Rhizomatous). Extra fine, large, flamed; very curious; not hardy.....................2 $235 \quad 1150$

IXIAS. These are extremely beautiful, fine for forcing and growing outdoors; not quite hardy, but can be arown in the garden with a little protection. 100 bulbs in 25 varieties.........................

Viridiflora. Green, black eye; extra. 85 ets. per doz., $\$ 6$ per 100

Mixed. Very fine mixture. 15 ets. per doz., 60 ets. per 100 .

L A C H E N L I A S, Mixed. $\$ 1.75$ per doz., $\$ 10$ per 100 .

LEUCOJUM æstivum (Summer Snowflake). 40 ets. per doz., $\$ 3$ per 100 .

Vernum (Snowflake). 30c. per doz., $\$ 1.75$ per 100 , $\$ 11$ per 1,000 .

MUSCARI botryoides album (White Grape Hyacinth). 20 ets, per doz., $\$ 1.40$ per $100, \$ 8$ per 1,000

Botryoides cæruleum (Blue Grape Hyacinth). 15 ets. per doz., 70 ets. per 100, $\$ 4.25$ per 1,000 .

Botryoides carneum (Pink Grape Hyacinth). Beautiful novelty. \$1.40 per doz., $\$ 8$ per 100 .

Botryoides pallidum grandiflorum. Pearl-blue. $\$ 1.75$ per doz., \$9 per 100 .

Conicum, Dark blue. 40e. per doz., \$2.75 per 100 .

Moschatus major (Musk Hyacinth). Grayish yellow; very fragrant. $\$ 4.25$ per doz.

ORNITHOGALUM Arabicum. $\$ 1$ per doz. \$2 per 100

Umbellatum (Star of Bethlehem). Lovely, and fine for naturalizing. $25 \mathrm{ets}$. per doz., $\$ 1.25$ per 100 $\$ 5.75$ per 1,000 .

RANUNCULUS, French. 100 in 25 varieties. $\$ 2$ per 100.

French, Fine Mixture. 20e. per doz., $\$ 1.20$ per 100 , $\$ 6$ per 1,000 .

Persian. 100 in 25 varieties. \$2 per 100 .

Persian, Fine Mixture. 20e. per doz., 65 ets. per 100 , $\$ 5$ per 1,000 .

Turban. Deep scarlet. 20c per doz., $\$ 1.10$ per 100 . $\$ 6.55$ per 1,000 .

Turban, Grootvorst, Bright searlet. 25 ets. per doz. $\$ 1.25$ per 100.

Turban, Hercules. Pure white. 45 ets. per doz. $\$ 2.75$ per 100 .

Turban, viridiflora. Crimson and rellow. 25 ets. per doz., $\$ 1.25$ per 100 .

Turban, Fine Mixed. $25 \mathrm{cts}$. per doz., \$1.50 per 100 , $\$ 9.50$ per 1,000 .
SCILLA campanulata alba. White............ Per doz. ${ }_{40}^{100} 1,000$

Campanulata cærulea. Blue............... 25 150

Campanulata rosea. Pink................... $40 \quad 25$

Hyacinthoides. Fine blue................... $30 \quad 150 \$ 1100$

Peruviana cærulea (Cuban Lily). Blue....... $160 \quad 1000$

Sibirica. Finest sky-blue. One of the loveliest

spring flowers; it has a color almost as dee as the sky itself. It is quite hardy, and flowers outdoors at the same time is snowdrops, in front of which it has a eharming effeet. It is fine for planting in the grass. If planted in pots and kept indoors, it may be had in bloom as early as Christmas. Its bright blue color makes it suitable for any decoration............. 18 18 $03 \quad 850$

200 SPARAXIS tricolor. Fine mixed .............. $20 \quad 75 \quad 400$

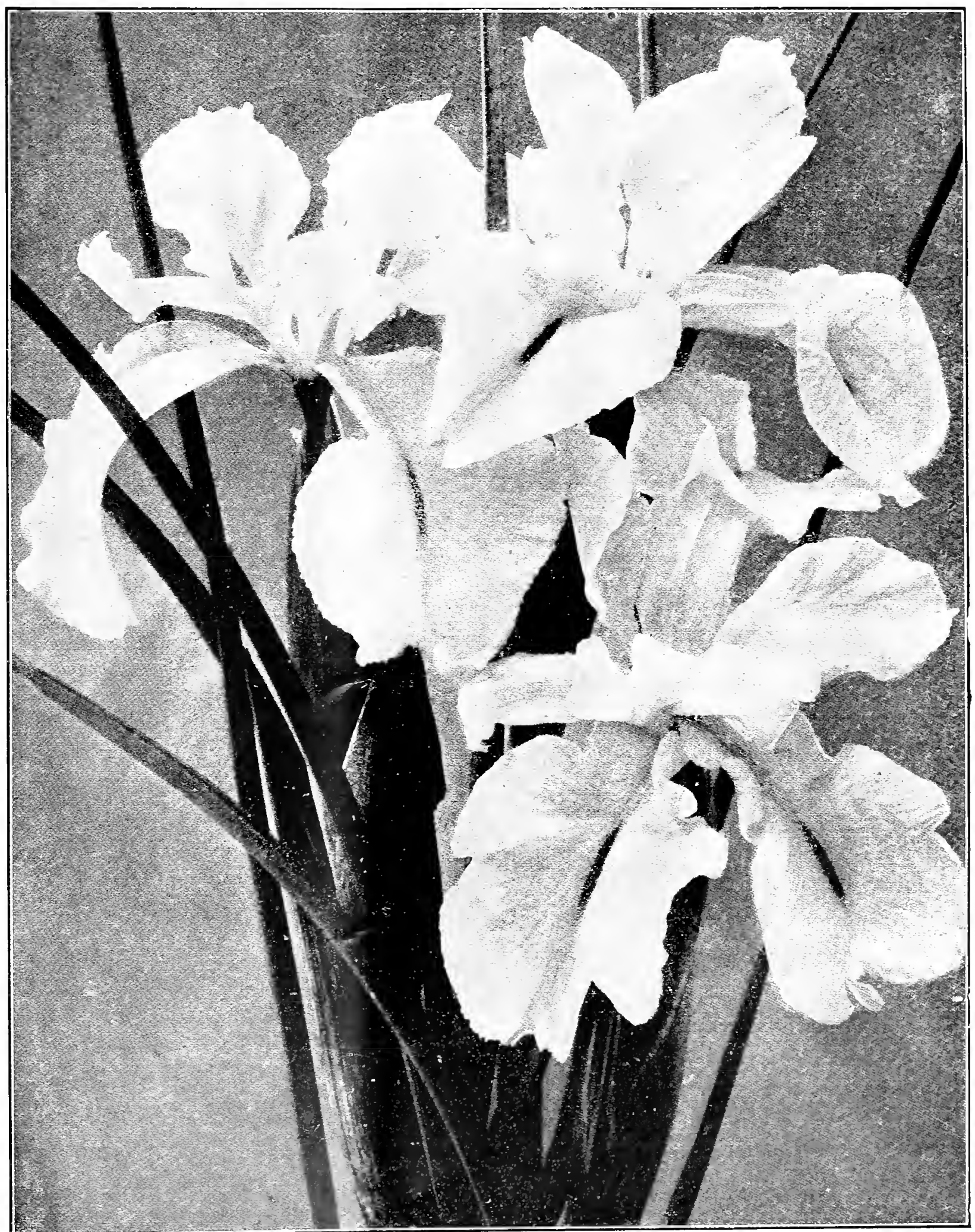

IR1S ANGLICA (See preceding páge) 


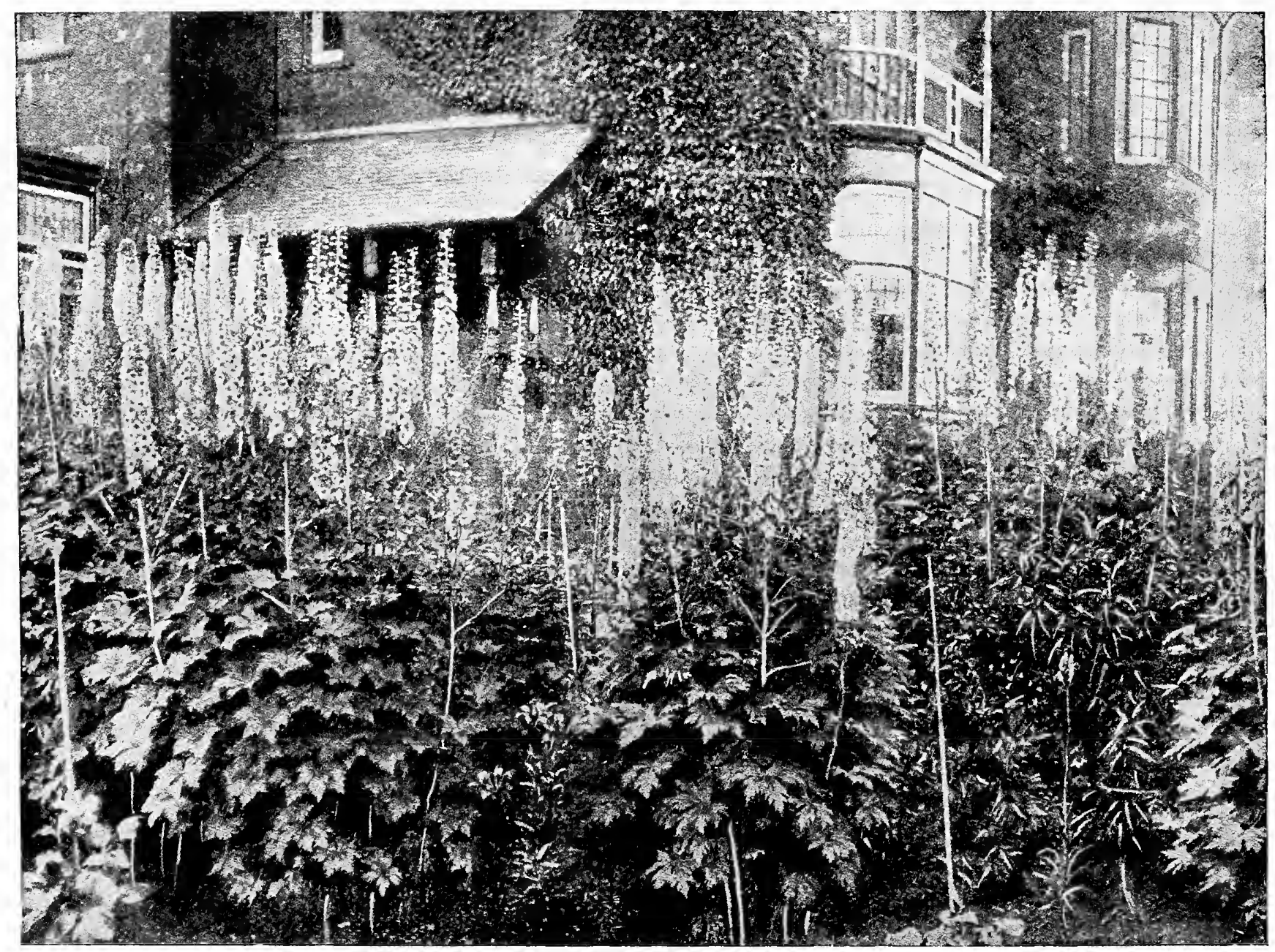

TALL ENGLISH DELPHINIUMS (From photograph)

\section{TALL ENGLISH DELPHINIUMS}

I am tempted to say that the Improved English Delphiniums are the most beautiful hardy plants in cultivation, but I am also tempted to say this of a score of other things, and, of course, it is impossible to say which is the most beautiful of hardy plants, for they have such an immense variety of beauty that the wonder grows that people continue to plant bedding plants by the million which have little or no beanty, are an annual expense, and cost quite as much as hardy plants, whose first cost is their only cost and which increase in size, in beauty, and often in quantity year after year. These Delphiniums may not be tle most beautiful hardy plants, but they are among the most beautiful, and nothing can be more distinct and satisfactory. They are stately and picturesque, some varieties growing eight feet high in rich soil; they have immense spikes of most beautiful flowers of every imaginable shade of blue, and their season is a long one ; in fact, they will bloom from spring till fall if properly treated.

Hitherto it has been impossible to get satisfactory varieties and plants of English Delphiniums ; in fact, no nursery in this country has had a satisfactory general stock of hardy plants, and I have had the greatest difficulty in getting the plants specified for my landscape gardening work, as this class of plants is extremely difficult to import. The difficulty became so great and my work was so hampered by the lack of plants to carry it out that I persuaded one of the most capable horticulturists in this country to start a hardy-plant nursery and guaranteed the financial results. It is the intention to make this nursery a model of its kind and grow everything in hardy plants worthy of culture and to send out nothing but well-grown plants packed in the most careful malner. The Delphiniums deseribed on next page are one of the offerings of this nursery (The Springdale Nursery).

CULTURE OF DELPHINIOMS. - The culture of Delphiniums is exceedingly simple, and the results out of all proportion to the slight amount of care necessary. They thrive in almost any position, and may be planted at any time of the year, provided that in summer the plants are not too forward, and that they be well-watered if the weather be dry. The soil may be a rich, friable loam, which suits them finely; but any soil, even hot and sandy, if well watered and manured, will give excellent results. Dig deeply-trenching is better-add plenty of well-rotted manure, and plant about $2 \frac{1}{2}$ feet apart. Placed in lines, as a background to a border, or in groups of, say, three plants at intervals, the effect of the Delphinium is exceedingly fine. They look well in beds also, arranged at the same distance apart each way. They are grand grown in masses of large groups of separate colors, and may be associated with shrubs with great advantage, succeeding by their robustness well in shrubberies. A succession of flowers may be expected from spring to early autumn, especially if the spikes. which have done flowering early be cut down to the ground; fresh growth will then be produced, which will give blossom. Copious watering in summer will be attended by increased size of spike and flower; in fact, in seasons of prolonged drought water is absolutely a necessity on many soils if the varieties are to exhibit themselves in their true size and beauty of flower and spike. Top-dressing is greatly recommended on certain soils, instead of the bare surface of the ground being left exposed to the sun. Some of the neater dwarf alpine and other hardy plants may be utilized to plant between and around Delphiniuins. Coal ashes strewn over the crowns will protect the plants from slugs through winter and spring. As we have intimated, any garden soil suits the Delphiniums; it is, however, necessary to secure sorts such as are offered below, in order to obtain an effect superior to that afforded by the old smaller-flowered varieties. No amount of liberal treatment will cause the smaller-flowered kinds of a few years back to develop into the gorgeous hybrids of today.

"The Delphiniums were the first thing that attracted my attention for they were just at their best and there was just about one acre of them. They niade such a sight as I shall not soon forget, so vast was the quantity in bloom, so grand the spikes, and so rich and varied the different shades of blue. Although I have been a grower of these lovely hardy border plants for some twenty years, I was not preparcd to see so much improvement in color, and was much struck with the intensity of the shade in many varieties as compared with the old Bella donna. The varieties which have a shade of bronze in the center are also very fine, the contrast between the bronze center and the deep blue erterior being very striking. But the shades of color in many of the newer sorts almost baffle description, and I shall not attempt it. It is in the doubles and semi-doubles that the greatest advancement has been made, and many of them are truly lovely. They are much larger and more compact than the old doubles of the Ranunculoides type, and consequently are much more valuable for ordinary garden adornment. The 
DELPHINIUMS, continued

light blue flowers with the large white eyes are very striking, and of this section Britannia is, I think, the very best Delphinium I ever saw. . . . The Delphiniums do not receive any special treatment here, being simply planted on a strong loamy soil in an open position. Some few were tied to stakes, but the majority do not require it, being so dwarf in comparison with the old sorts. This dwarfness of habit is a great gain, and the greater portion of the plants were not more than 4 to 5 feet high, yet carrying immense spikes of large bloom."-The Gardening World.

\section{SPECIAL OFFER OF IMPROVED ENGLISH DELPHINIUMS}

I want every one who receives this price-list to try these improved English Delphiniums, and to that end offer them at extremely low prices. I guarantee that they will give unqualified satisfaction in every instance and will be a revelation of beauty to most people.

Fine Mixed English, grown from seed of Kelway's famous named doz. 100

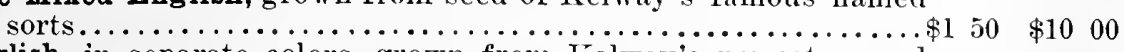
English, in separate colors, grown from Kelway's newest named

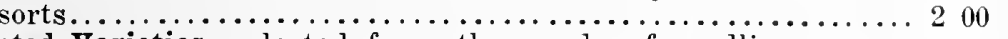

Selected Varieties, selected from thousands of seedlings grown

from Kelway's choicest named sorts .................... $300 \quad 2000$

Extra Selectod Varieties.......................each, 50 ets... 500

White Varieties.............................each, $\$ 1.50 \ldots$

\section{Delphinium formosum}

In the perennial Delphiniums or Larkspurs we have one of the most beautiful and useful families in hardy plants. The old Delphinium formosum is one of the few hardy plants which has been pretty generally offered by the plant trade; yet it is comparatively unknown, although few things can be planted that will give more satisfaction, whether the object is decorative effect in the garden or flowers for cutting for the house. Messrs. J. H. Small \& Sons, the leading florists of New York and Washington, have nade quite a hit with it as a novelty in cut-flowers. The flowers are intense deep blue, perhaps the nost brilliant blue to be found in flowers freely produced in long spikes; it blooms in June and July and continues for a long time in bloom, and if the plants are cut back after flowering will bloom a second time in the fall. Plants grow to 4 or 5 feet high, and if planted in masses about two feet apart will produce a splendid effect. $15 \mathrm{cts}$. each, $\$ 1.25$ per doz., $\$ 8$ per 100 .

\section{Delphinium formosum cœlestinum}

This rare and scarce Delphinium has all the good qualities of the well-known Formosum, but instead of dark blue flowers produces great spikes of exquisite light blue bloom, the loveliest shade of blue in the floral world. I have always treasured this Delphinium in my garden, but could never get enough stock of it to offer. This season I have secured three thousand plants, which I can offer at a reasonable price. $20 \mathrm{cts}$. each, $\$ 2$ per doz., $\$ 12$ per 100.

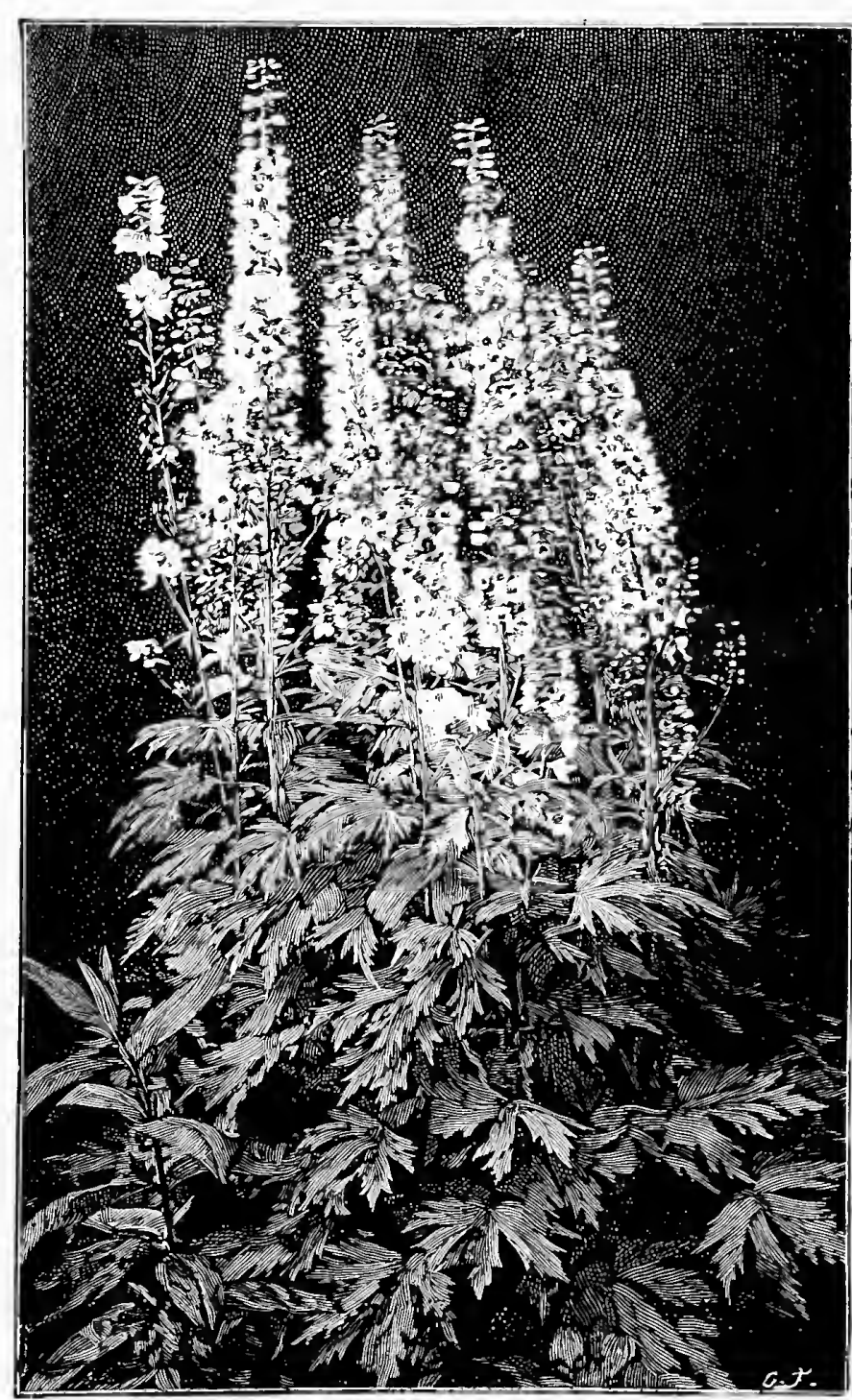

SPECIMEN PLANT OF IMPROVED ENGLISH DELPHINIUMS

\section{Formation of Lawns from Seed}

The ground should be thoroughly drained and well prepared. The soil ought not to be too rich, as a rapid growth is not wanted in the grasses of a lawn, but the surface should be as much alike in quality as possible. After sowing, the ground should be rolled, in order to press the seed firmly into the soil. The proper time to sow grass seed depends, of course, upon the latitude. In tbe central and eastern states, from September 15 to October 15 is the best time. Seed may also be sown in the spring, provided it is done early enough to secure a good, strong growtl before hot, dry weatler of summer sets in. 'The sowing should be done when the ground is moist, or before an expected rain, and a subsequent rolling is always advisable.

Rolling. - As soon as the frost is out of the ground in the spring, the land should be gone over with a heavy roller. Winter frosts loosen the soil, and rolling is necessary to compress it again. lf grass seed is to be sown, this should be done first and the rolling inmediately afterward. Frequent rollings ore recommended.

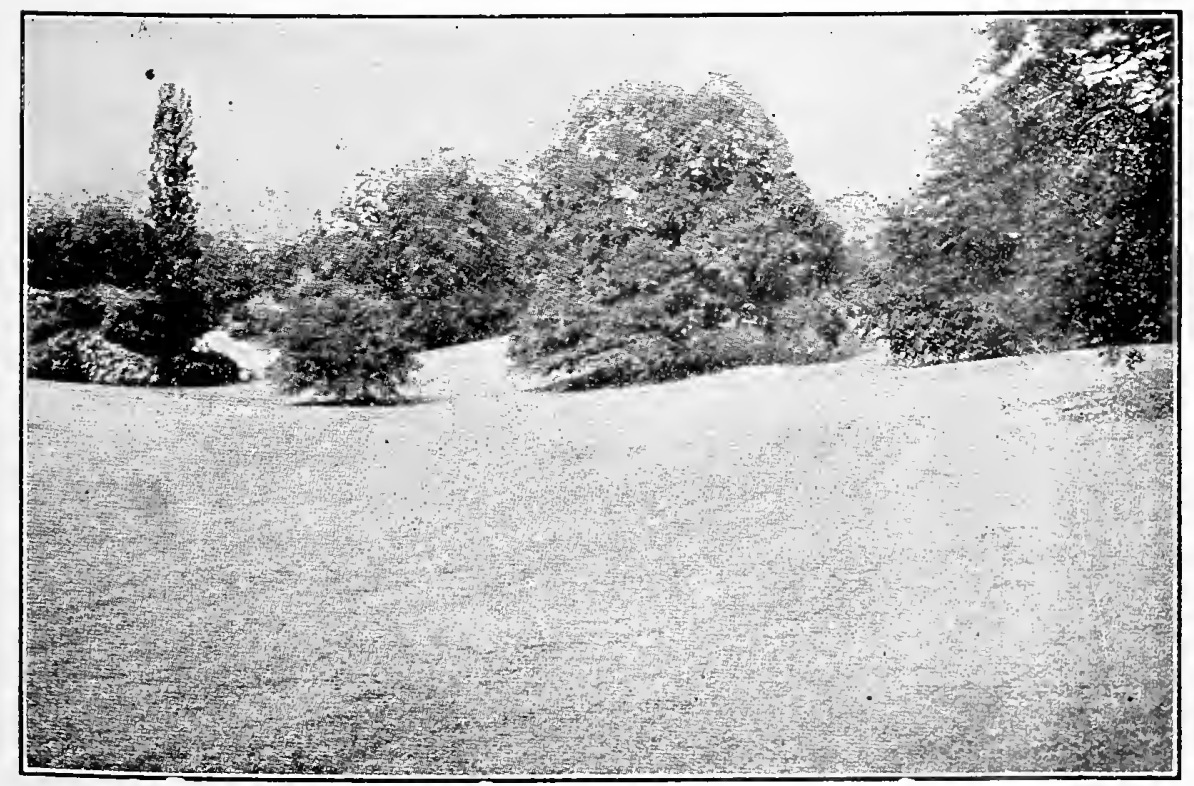

Mowing, - All turf-forming grasses are improved, both in vigor of root-growth and fineness of texture, by frequent mowings, It is impossible to say just how often the grass should be morn, as that depends upon the rate at which it grows. Too close cutting should he guarded against, however, especially during the hot sumner months, when the roots require some top-growth to protect them from the burning sum. A good top-growth is also necessary to protect the roots from the serere winter frosts. Mowing should, therefore, be discontinued in time to let the grass grow pretty long before winter sets in.

\section{PRICES OF GRASS SEED}

We can supply the very best quality of Mixed Lawn Grass Seed for $\$ 2.50$ per bushel. This is exactly the same quality of seed that is usually sold for $\$ 5$ per bushel as Central Park Mlixture, or under some other fancy name. Grass seed weighs only 14 pounds per bushel, and can be shipped inexpensively by express. We do not supply less than one-half bushel. Special prices quoted for large quantities. Grass seed is sold by weight, 14 pounds for a bushel, but 14 pounds of clean grass seed will not fill a bushel. Grass seed can be sown advantageously in the fall, preferably in September. Prices: 1/2 bushel, $\$ 1.50$ 1 bushel, $\$ 2.50$; 10 bushels, $\$ 2.25$ per bushel; 25 bushels or over, $\$ 2$ per bushel. Small quantities shipped from here; large quantities from Cincinnati. 


\section{Hardy Perennial Phloxes}

The beauty and usefulness of these grand border plants give to them a deservedly first place among hardy plants. For cutting, their large trusses go a long way in floral decorations. In color they range from pure white to the richest crimson and purple, and from soft rose and salmon to bright coral-red, all having a delicate fragrance. Perennial Phloxes succeed in almost any soil enriched with manure, but are much benefited by a mulching of decomposed manure in spring, and in hot weather an occasional soaking of water. If the frst spike of bloom is removed as soon as over and the plant given a good soaking of water, they will produce a second supply of flowers, thus continu. ing the display until late in autumn. These later blooms are often finer than the first.

The following varieties are a selection made by me in France, Germany, Holland and England, and include all the very best of the new and old varieties.

\section{Field-grown plants ready for fall delivery, except where noted, 15 cts. each, $\$ 1.50$ per doz., $\$ 10$ per 100}

Aurora. Brilliant orange-crimson, bright crimson eye; a very showy variety.

Adonis. China-rose, large carmine eye.

Argon. Light purple, shaded white; very distinct. 25 cts.

Athis. Tall; salmon. The tallest-growing of all Phloxes.

Bacchante. Crimson, with carmine eye; dwarf and rery bushy; full-flowering.

Beranger. Rosy white, suffused gray; very delicate shade.

Berenice. Pure white; dwarf and bushy. A lovely sort. $25 \mathrm{cts}$.

Boule de Feu, Bright rosy red, dark center.

Captain Wilhelmy. Dark erimson.

Charlotte Saison. White, richly shaded with purple-rose; extra tall. Coquelicot. Orange-scarlet, center purple; magnificent color for bedding. The most brilliant Phlox yet produced.

Comet. Rich dark crimson; the richest colored of all Phloxes. 25e

Eclaireur. Bright purplish rose, light center; enormous flowers.

Embrasement. Salmon-scarlet; extra fine.

Esperance. Pale mauve, fine white eye: extra fine.

Eugene Danzanvilliers. Light lilac, large white eye; immense trusses

Ferdinand Cortez. Bronzy copper; distinct and fine.

Fiancee. The best white; enormous pyramidal spike. 25 cts.

Frau Ilona Von Barczay-Waldeck. Pure snow-white; very large. 25c. Frau S. Buchner. Salmon-pink; large and fine.

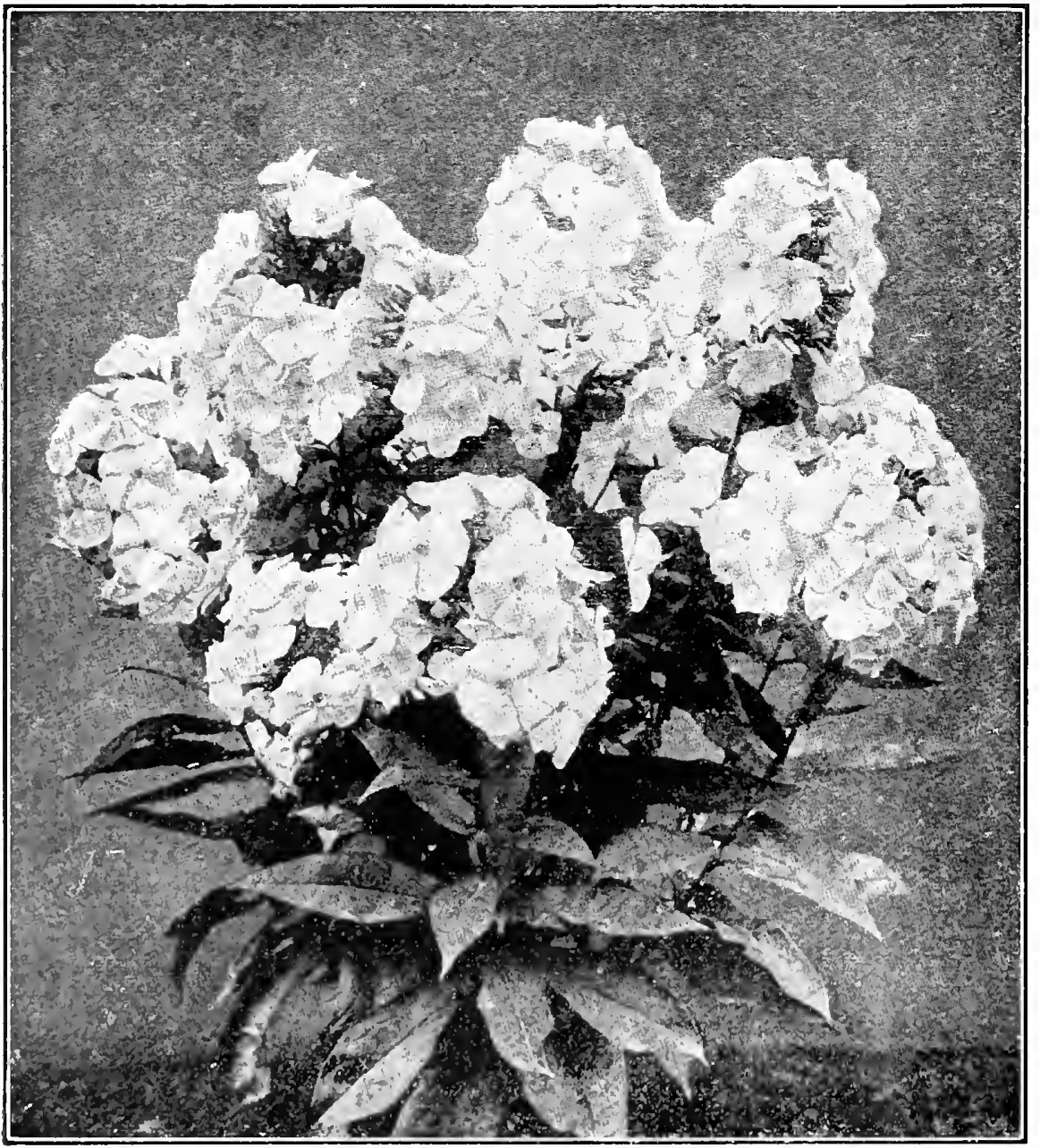

PERENNIAL PHLOXES
Gartendirector A. Medin. Bright lilac-carmine, flamed with white white eye.

Georges Bruant. Carmine, with large white eye.

General Breart. Blush white, with large carmine eye; $21 / 4 \mathrm{ft}$. high. Gloire d'Orleans. White, rosy purple center; variegated foliage.

Henri Murger, Purest white, with deep rose center ; large, handsome truss; $13 / 4$ feet high. 25 cts.

H. 0. Niger. Pure white, large crimson eye.

Kossuth. Deep rose.

Liberte. Lovely soft cerise-salmon; large, branching spikes; extra fine. $25 \mathrm{cts}$.

La Neige. Pure white.

La Candeur. Pure white.

Lothair. Crimson.

Le Mahdi. Steel blue; very intense color. An award of merit for this variety August, 1899 .

Le Siecle. Rose, white eye; distinct.

Le Soleil. Lovely soft rose-pink, with white center; a charming color; extra fine; 2 feet high. 25 cts.

Marquis de Breteuil. Bright pink, light center; very fine; $1 \frac{1}{2}$ feet high.

Miss Lingard. The best Phlox in cultivation. It produces immense heads of beantiful white flowers in June, and blooms again in September and October. Splendid foliage and babit and free from attacks of red spider.

Miss Cook. White, pink eye; early.

Hadame Pope Carpenter. White.

N. Smith. Dark crimson.

Obergartner Wetteg. Light rose; center lighter, with red eye.

Orientale. Rosy mauve, white center; fine trusses of large flowers.

Pearl. Pure white; very late.

Pantheon. Salmon-rose; a splendid variety.

Richard Wallace. White, pink eye.

Schlossgartner Reichenauer. Pure white.

Splendens. Brilliant glowing crimson; flowers small, but color rich; $21 / 2$ feet high.

Undulata variegata. Variegated foliage.

\section{NEW PHLOXES}

The following Phloxes were raised on $\mathrm{my}$ own grounds, and I can commend them as being distinct and fine. Field-grown plants ready for fall delivery.

25 cts, each, $\$ 2.50$ per doz.

DANSKE DANDRIDGE. Light purple, large star-shaped white center, sometimes mottled purple and white; a distinct and lovely variety.

CHESWICK. Salmon, bright crimson center.

SPRINGDALE. Deep pink, dark center; fine.

MARGARET ELLIOTT. White, red center; extri large panicles; very showy.

JEAN. Deep pink, large bright red eye; very distinct.

ROSALIE. White, blush center; delicate and distinct.

DEFIANCE. Bright deep red, almost as briglit as Coquelicot and much hetter habit.

MARGARET SLACK. Bright pink, red center; dwarf and hushy: loose panicles; very free-flowering. 


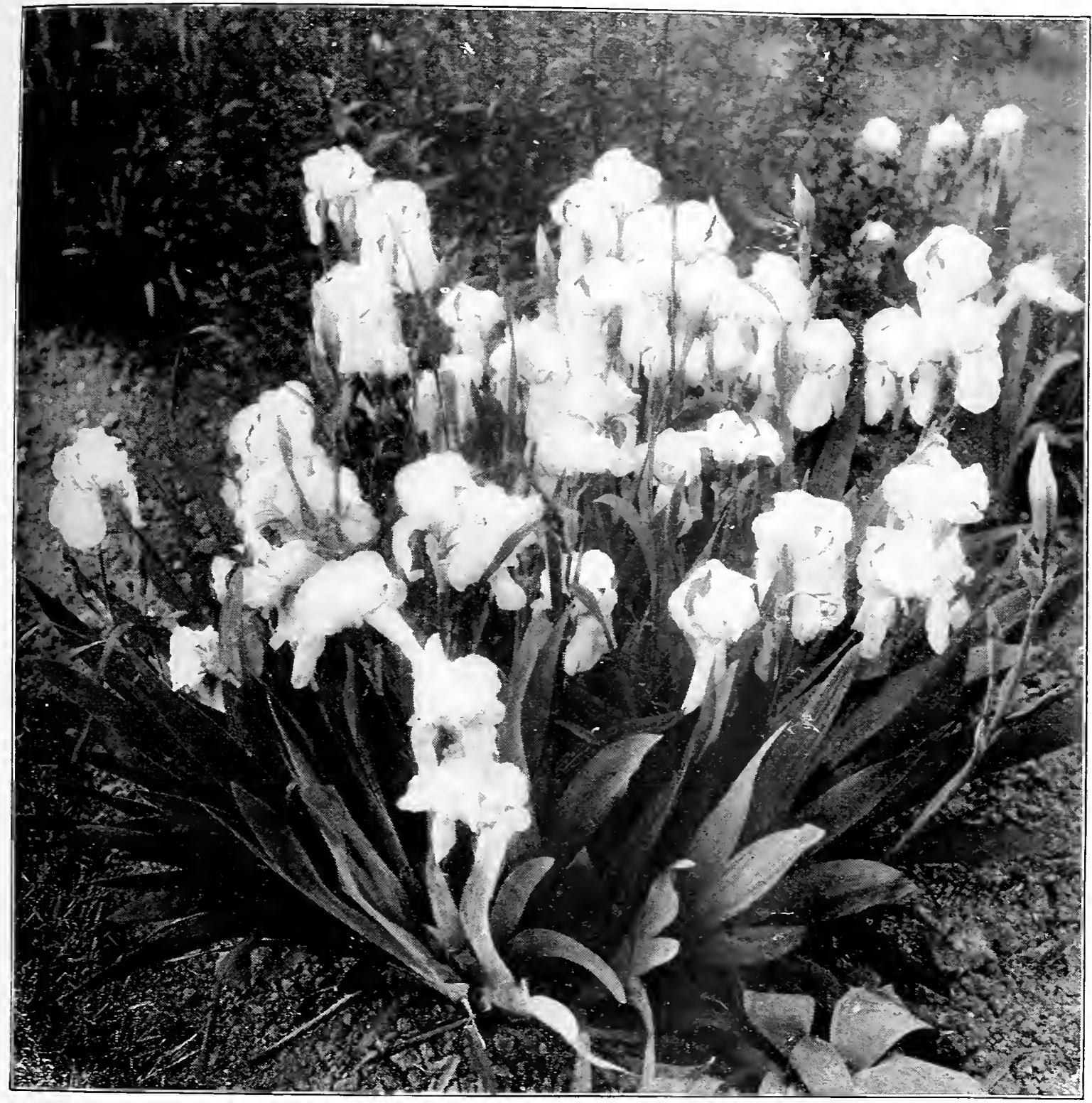

GERMAN IRIS

\section{GERMAN IRIS}

In the Iris family the German rarieties rank second in importance, the magnificent Japanese Iris being firt, of course. They bloom profusely early in May, are of the greatest hardiness and easiest culture and should he freely planted in erery garden. These Irises are the "Flags" of the oldfashioned gardens. They are most effectire when used as edging for a shrubbery or garden border There are no pure white rarieties

Named Varieties. 15 cts. each, $\$ 1.25$ per doz, $\$ 8$ per 100 , unless otherwise noted.

Mized Varieties. 10 cts. each, 75 cts. per doz., $\$ 5$ per 100 .

Celeste. Delicate larender.

Canary Bird. Pale yellow; lorely.

Florentina. Silvers white; early.

Honorable. Sellow, falls brownisli maroon.

Madam Chereau. Pearly wlite, daintly edged with lavender: one of the best.

Pallida speciosa. Larender with rosy tinge; fragrant; lovely.

Silver King. Silvery white; lorely. Queen of May. Lorely soft roselilac, almost pink. $20 \mathrm{cts}$. each, $\$ 2$ per doz.

Auralia. Purple.

Annette. Tellow, purple-maroon falls.

Edith Cook. Yellow, maroon falls. Parisienne. Deep purple; drarf. President Thiers. Bronze-purple, dark purple falls.

Vesta. Deep sellow, maroon falls.

\section{JAPANESE IRIS}

Some rears ago a set of Japanese Iris ( $I$. Kempferi) were sent to us from Japan to test. which were said to be identical with the collection $: n$ the Royal Gardens. "Te cannot rouch for this statement, as we have nerer been in Japan, but we hare nerer seen another colleetion in America or Europe that would equal it in ans war. The collection contained mans colors and rarieties we had nerer seen before, and the flowers were of remarkable size and beauts. When these Irises were in bloom thes excited the greatest admiration and enthusiasm. and it was bard to conrince people that these unique and exquisitely beautiful flowers were as hardy as apple trees. and as easily grown as potatoes. Ther will thrive in any good garden soil, but if the soil is made rerr rich and deep. and flooded with water for a month before and during their blooming season, ther will produce flowers of a wonderful size, sometimes 10 to 12 inches across. These Irises should be planted in full exposure to the sun. As the Japanese names are unintelligible and imposslble to remember. We hare renamed this collection.

Since the abore collection was receired we hare annually added to it the newest rarieties from Japan and new rarieties selected from. thousands of seedlings grown on our own gromds, until we now have what is unquestionably the finest and most complete collection of Japanese Iris in the world.

\section{PRICES OF JAPANESE IRIS}

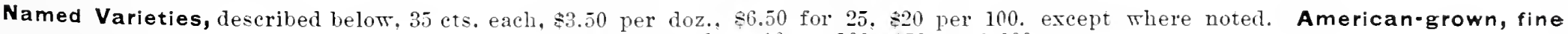
mixed, $\$ 1.25$ per doz., $\$ 6$ per 100 . \$50 per 1,000 .

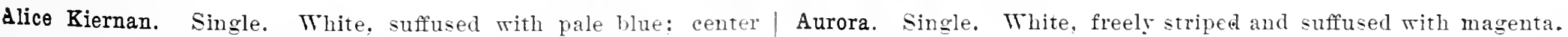
white, edged blue; extra large and fine; desirable. 5 cts.

Alvin C. Spindler. Single. Dark blue. striped and bloteled with reddish plum; center maroon. edged white: rery livelș, robust and rigorous plants inclined to spolt. $75 \mathrm{cts}$.

Allegheny. Double. Pale larender, striped white: late.

Amoret. Double. Blue, blotched and mottied with dark blue and reddish plum, sellow bar at base; late. $75 \mathrm{cts}$.

Angelo. Double. Bluish purple, center of petals white with rellow band at base.

Apollyon. Double. Reddish plum, striped white. base of petals 5ellow; large and fine. 60 cts.

\section{5 cts.}

Bessemer. Double. Bright blue: distinet. 1.

Blue Flag. Donble. Indigo-blue. base of petals rellow: the last rariety to bloom. $\$ 15$ per 100 .

Coronet. Double. White ground, densely spotted and blotched with rose and maroon. \$1.

Cærulea. Double. Larender-blue, delicate coloring: large and distinct. $\$ 1$.

Coquette. Double. IIagenta, rellow center feathered with lilac; inclined to sport: distinct.

Cygnet. Single. Purple and plum, blotched white. 


\section{JAPANESE IRIS, continued}

Delight. Single. Dark rose, yellow spot, purple at base of petal. 60e. Dick Webb. Double. Rose, yellow band at base of petals; very distinct. 75 ets.

Distinction. Double. Extra large flowers; base of petals yellow, blotched and striped white and magenta; distinct. 60 cts.

D. s. Newhall. Very double. Bright blue, center white; distinct; extra fine. $\$ 1$.

Elizabeth. Double. White, sometimes slightly penciled blue. $75 \mathrm{c}$.

Enchantress. Single. Pale blue, penciled with dark blue.

Esmeralda. Single. Magenta, penciled white, center white.

Fairy Queen. Single. White, base of petals yellow, maroon center. Gigantea. Double. Bluish purple, lightly striped white; vigorous grower and free bloomer. The earliest-flowering variety in the collection.

Glow. Double. Velvety maroon; large. \$1.

Heart of Gold. Double. Extra large white flowers, yellow center.

Hermione. Single. White, maroon center, petals penciled with blue.

Ida. Reddish plum, rich and velvety; narrow yellow band on base of petals.

James R. Mellon. Double. Extra large flower; lilac, striped with purplish blue, purple center; distinct and fine. $\$ 1$.

James F. Parker. Double. Rich dark blue, yellow center; large and fine. 75 cts.

John Marron. Double. White, distinctly penciled with blue; blue center, base of petals yellow; late.

John C. Slack. Double. Lilac, heavily blotched with magenta and blue; yellow center; distinct. $\$ 1$.

King of the Purples. Single. Dark rich purple; extra large and fine; very striking. $60 \mathrm{cts}$.

Kirk. Very double. Crimson-maroon, rich and velvety, petals striped lightly with white. $50 \mathrm{cts}$.

Kitty. Single. White flower, slightly suffused with pale blue. 75 cts.

Laura. Double. White, penciled dark blue; purple center; Iate.

Lorna Doone. Double. White, delicately penciled with blue; yellow band at base of petal; blue center.

Mary Anderson. Double. Light blue, blotched with dark blue; yellow spot at base.

Merry Hampton. Single. Reddish plum; base of petals yellow, with broad band of white. 60 cts.

Mikado. Rich glowing purple. Royal in its beauty and effect. 75 cts.

Minerva. Double. Bright blue, striped and blotched white. Exceedingly handsome. 60 cts.

Mont Blanc. Double. Pure white; large and fine. One of the finest of the white varieties.

Moonlight. Double. White flower, with yellow center. Rich and dainty in effect.

Mrs. James H. Ballantine. Single. Extra large wlite flower, delicately suffused with pale blue; margin of petals pure white; fine.

Mrs. Alexander King. Double. Lavender, edged reddish purple; yellow spot at base of petals; extra fine. 75 ets.

Mrs. Henry S. Turner. Double. Reddish purple; center of petals bright blue, with large yellow band; extra strong grower; distinet and slowy.

Mrs. D. E. Richardson. Double. Rose, shading to white at margin; center white; very distinct. Ons of the best of the new sorts. \$1.

Mrs. R. H. Boggs, Single. Pale blue, blotehed white; large, distinct and fine. $\$ 1$.

Mrs. Frank H. Hiscock. Double. Delicate blush; dis tinet and lovely. $75 \mathrm{cts}$.
Mrs. Henry L. Higginson. Single. Bright reddish maroon, cen. ter white.

0ctavia. Single. White, dark center; petals distinctly netted with blue.

Queen of the Whites. Double. White, vigorous grower.

Regina. Double. Blue, striped white and edged reddish purple. 60c.

Romola. Single. Lilac, striped witl plum; plum center.

Rosalind. Double. Light purple, freely striped with white.

Sewickley. Single. Purple, blotched and mottled with white.

Snowdrift. Single. Pure white.

Springdale. Single. Bluish purple, slightly striped white. 75 cts.

Venus. Single. Lilac, freely striped and suffused with purple.

Westmoreland. Double. Light blue, striped and suffused with darker blue. 75 ets.

W. J. Buttfield. Double. Center of petals white, heavily margined with magenta; vigorous grower. $\$ 15$ per 100 .

Wm. J. Matheson. Double. Reddish plum; base of petals yellow; large and fine.

Wm. Falconer. Double. Rich royal purple; extra fine. 50 cts.

Wm. A. Procter. Double. Dark blue, slightly striped white; yellow center. $\$ 1$.

Wm. F. Dreer. Double. White, penciled with lavender. $\$ 1$.

Yokohama. Double. Reddish purple; base of petals yellow; large and fine; late. $75 \mathrm{ets}$.

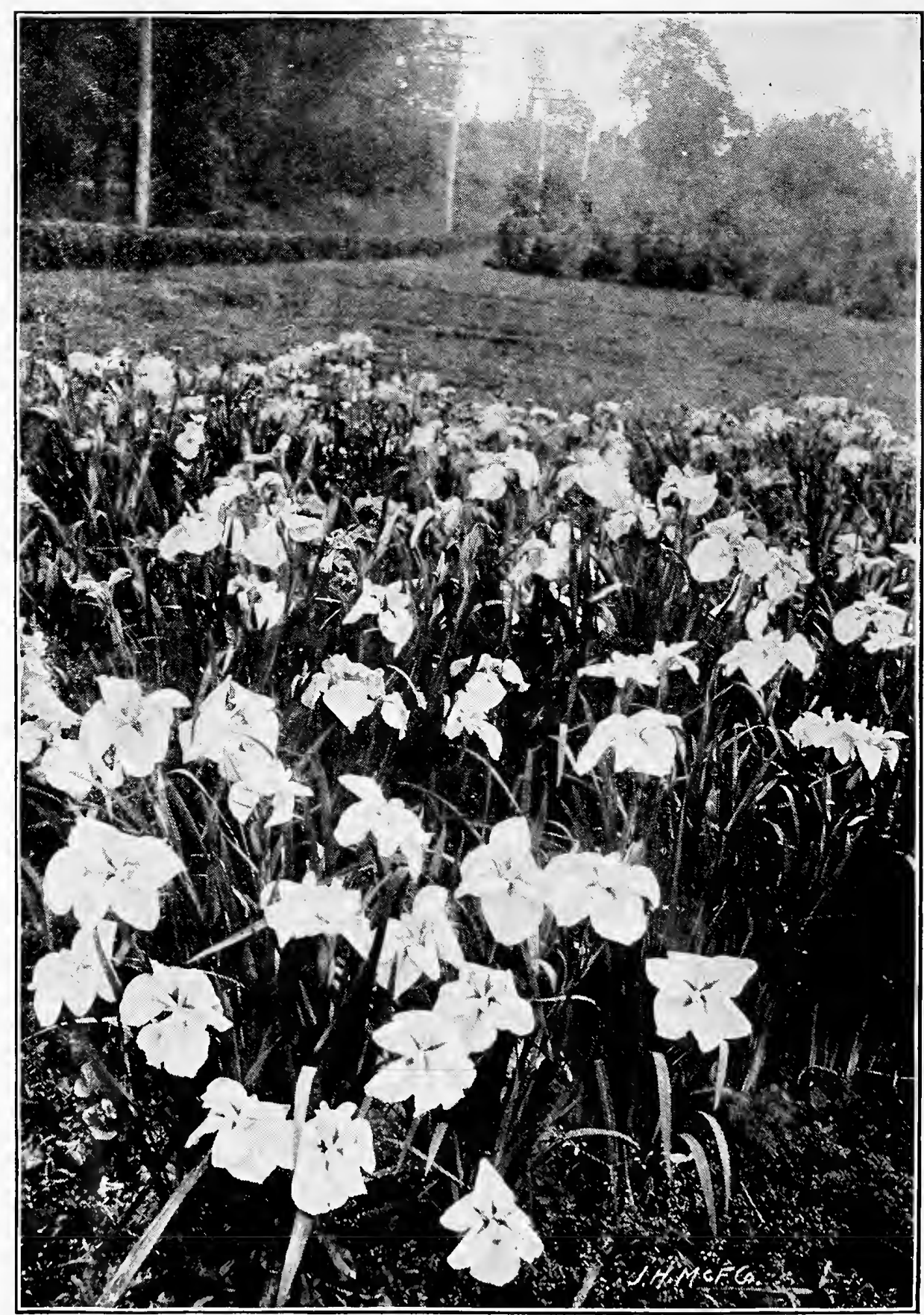

GROUP OF JAPANESE IRIS 


\section{TREE PEONIES}

Our stock of these splendid Shrubby Peonies is the largest in America

The Japanese varieties are the most beautiful but the most diffi. cult to grow on account of suckering. The European varieties are grafted on herbaceous roots and do not sucker.

\section{JAPANESE VARIETIES}

Fine blooming plants (grafted) in 25 choice varieties, $\$ 1$ each, $\$ 10$ per doz.

Extra selected blooming plants in 25 choice varieties, $\$ 1.50$ each, $\$ 15$ per doz.

Japanese Tree Peonies on their own roots (supply limited), large specimen plants, probably 20 years old, $\$ 10, \$ 15$ and $\$ 20$ each, according to size.

\section{EUROPEAN VARIETIES}

Best Named Varieties, 2 years old..............\$1 50 Each Perdoz.

. " "

5 years old.............. $300 \quad 3200$

Queen Elizabeth. One of the best European rarieties.

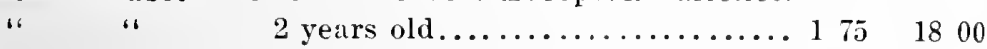

4 years old ................. 250

5 years old ................. $325 \quad 3600$

Moutan. A fine old pink variety............. $75 \quad 800$

In ordering Tree or Herbaceous Peonies, please state if it is desired to have them shipped as soon as ready in August or September.

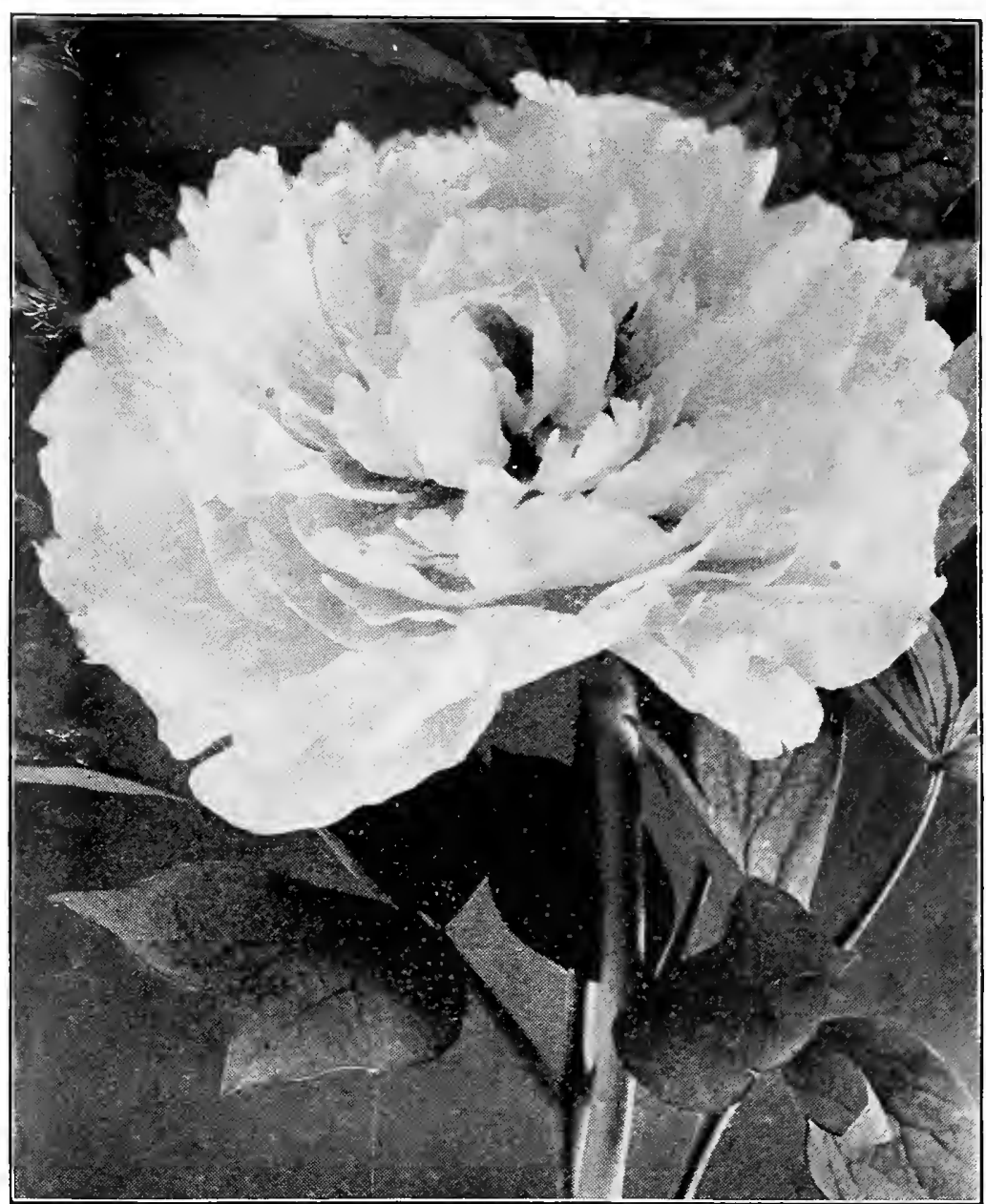

MOUTAN TREE PEONY

\section{HERBACEOUS PEONIES (Ready in September)}

It gives us great pleasure to see the increasing popularity of Peonies, for there is nothing more deserving, and when the merit and

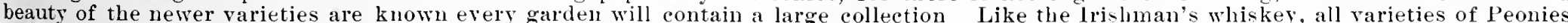

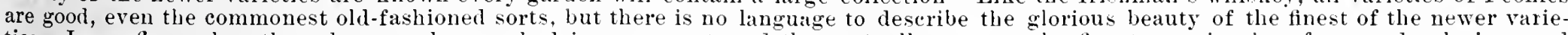

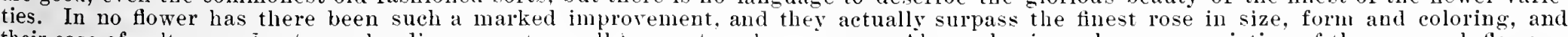

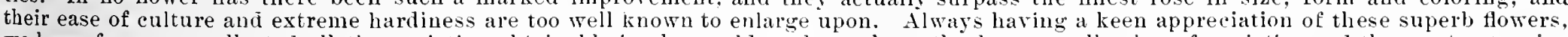

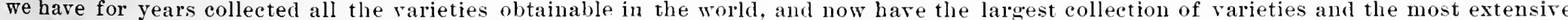

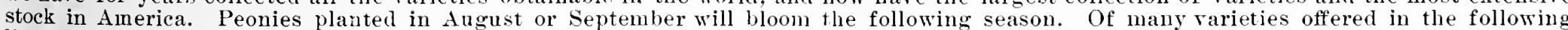

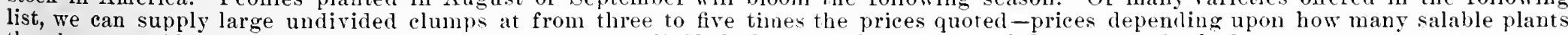
the clump would inake if divided. By planting these undivided clumps a fine display of flowers can be liad next season.

\section{JAPANESE VARIETIES}

Double and Semi-Double. In 25 varieties. These Each Perdoz, 100 are really very choice and distinct from varieties grown in this country, and will give the greatest satisfaction .................... \$0 60 $\$ 600 \quad \$ 1500$

single. In 25 varieties. The finest Single Peonies undoubtedly come from Japan. They are equal or superior to single sorts coming from Europe costing three times as much ........ 70 $750 \quad 5500$

\section{CHOICE NAMED VARIETIES}

Abel Carriere. Good-shaped Howers, anemone-fawu, Each Per doz. amaranth-violet......................... $\$ 035 \$ \$ 350$

Agida. Brilliant red; very free-flowering.......... $35 \quad 350$

Alpha sulphurea. Very full, grand, globular Hower; beautiful shape, pure white, center sulphur-yellow; extra

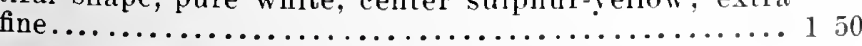

Armandine Mechin. Large Howers in clusters; rery brilliant clear amaranth $\ldots \ldots \ldots \ldots \ldots \ldots \ldots \ldots \ldots \ldots \ldots$

Arsene Meuret. Large, globular flower of fine shape; beautiful lilac, with clear violet border..............

Arthemise. Large flowers; lovely soft rose and salmon; very beautiful .......................... 100

Asa Gray. Large, full flower, imbricated, beautiful form; carnation-salmon, powdered with carmine-lilac. One of the best ............................ 150

August Lemoinnier. Large anemone-formed flower;

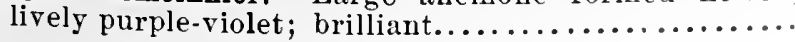

August Miellez. Large flowers, in clusters; clear lilac Euch Per doz. and purple, center chamois and lilac...........\$0 40 \$4 00

Baron J. Rothschild. Outside petals rose, center salmon;

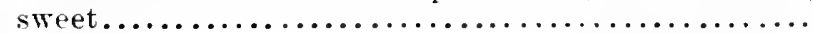
Ba ard. Beautiful clear brilliant violet............

Belle Douaisienne. Large, rery full, imbricated flowers; carnation and chamois, chalice streak of carmine; ex-

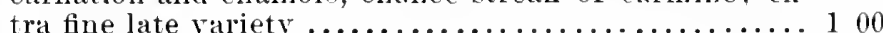
tints; late-flowering; extra .................... $100 \quad 1000$
Blushing Bride. Light rose ................. 50

Buyckii. Large, globular flower: lovely rose, center salmon-rose with silver reflex: extra fine........... Blush White. A good variety, with blush flowers, turning white after opening.............per $100, \$ 14$.

Compte d'osmont. White, center sulphur............ Cameron. Brilliant purple-violet, shaded with relvet Candida flore pleno. Dark red, rosy white center; fine.. $125 \quad 1200$ Candidissima. Beautiful anemone-formed tlowers, very tull, clear sulplur-yellow, with green heart; extra....

Carnea alba. Large flowers; clear carnation, center

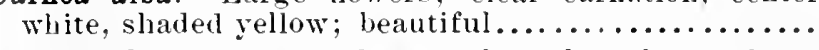
Carnea elegans. Fine flower of perfect form; large petals, clear carnation with satin reflex mixed with small yellow petals, very fresh coloring; fine........

Caroline Allain. Beautiful blush, center sulphur, tip-

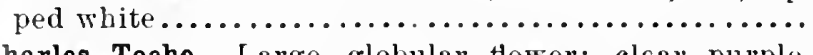
charles Toche. Large, globular tower; clear purple, with carmine reflex, golden stamens. Elegant variety. 100

500

75 


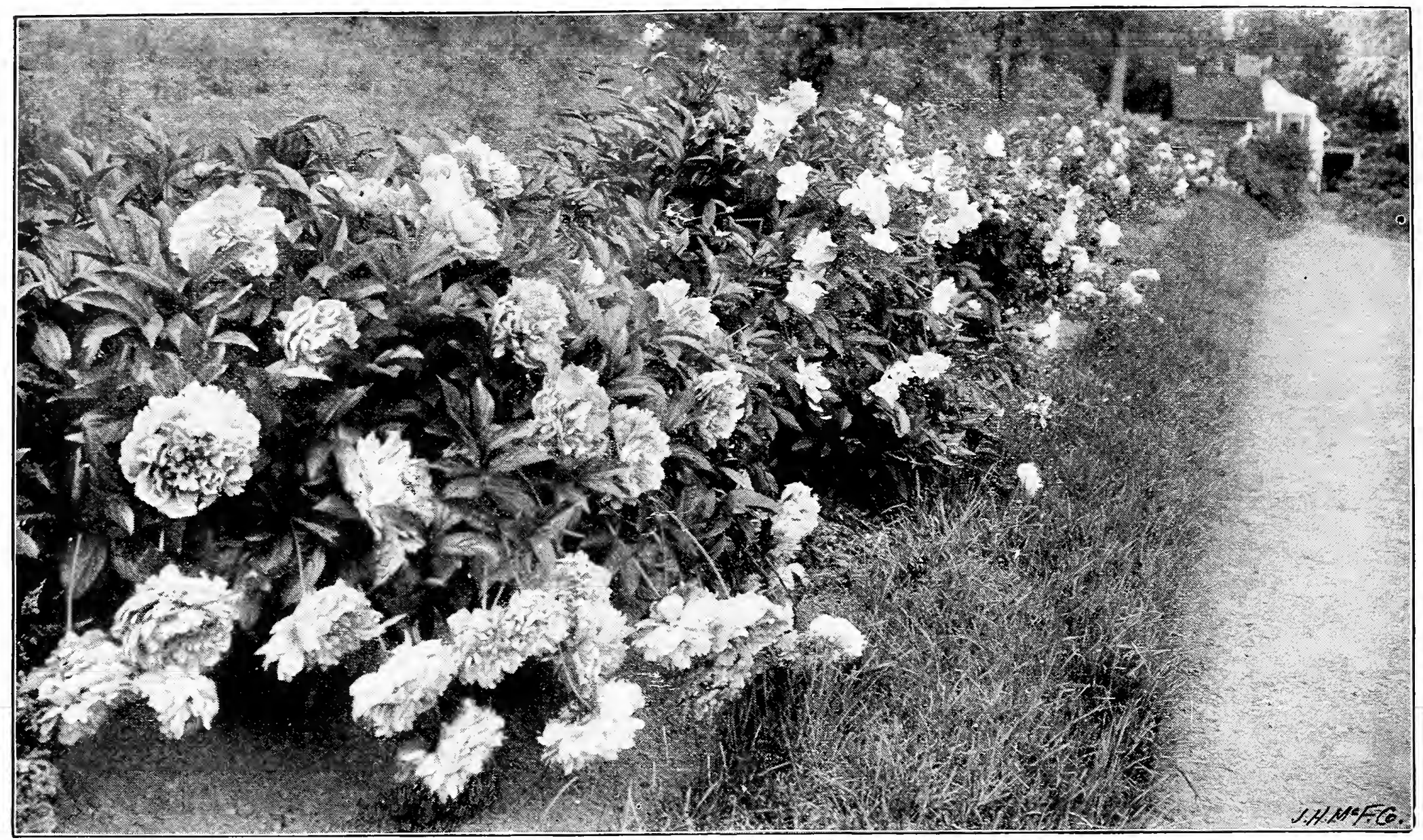

BORDER OF HERBACEOUS PEONIES

CHOICE NAMED PEONIES, continued

Comte de Paris. Rose-color, center salmon-yellow, crested with large petals of lovely rose; very beautiful variety ..........................\$0

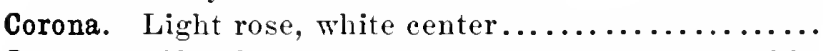

Couronne d'Or (Golden Crown). Large, imbricated white flower, yellow reflex with stripes of carmine aud

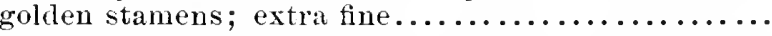

Curiosity. Large petals of clear violet-red, those of the center transformed into golden ligules; very distinet

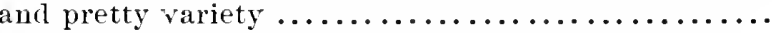

Carlotta Grisy. Large purple flower, shaded carmation-

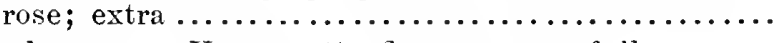

Charlemagne. Very pretty flower; very full; cream-

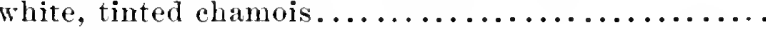

Charles Binder. Beautiful lively deep rose; equally

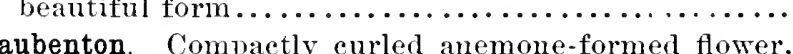
lilac-purple rose, white edge; very beautiful.......

Delachii. Large, eup-shaped flower, deep amaranth,

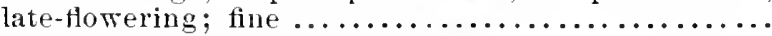

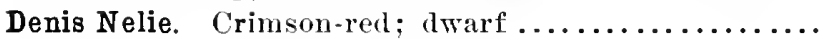

Descartes. Very large flower; brilliant clear amaranth.

Doyenne d'Enghien. Violet-rose and earmine.........

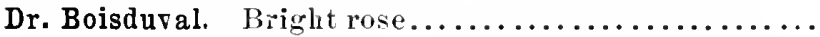

Dr. Bretonneau (Verdier). Large, globular flower: large rose petals and clear white; beautiful ........ Duc de Cazes. Large petals of lively carmine-red, cen-

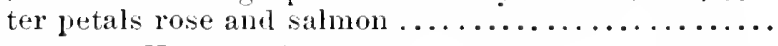

Duchesse de Nemours (Verdier). Rose-piuk: very large.

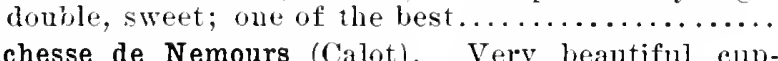

shaped flower, sulphur-white with greenish reflex, pretty bud.............................. 100

Duchesse d'0rleans. Beantiful carmine, rose center, in-

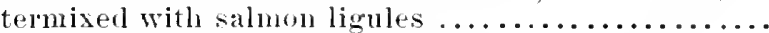

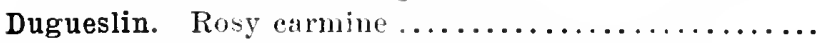

Duke of Wellington. White, with yellow center .......

Dorchester. Cream color, tingerl pink; very double; fragrant ..........................1 00
Each Perdoz.

$\$ 350$

$40 \quad 400$
Eclatant. Bright carmine; large flower............. Each Per doz. Edel Kanig. Deep rose ....................... 25 250

Edouard Andre. Large, globular flower. Deep crimson-red shaded black, with metallic reflex; stamens golden yellow, magnificent coloring, producing a grand effect ............................... 300

Edulis alba. Large white flower, with some stains of carmine in the center; very pretty variety ..........

Edulis superba. Very large flower of perfect shape; beautiful brilliant tinted violet mixed with whitish

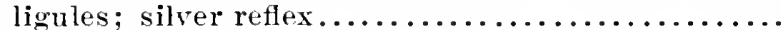

Epicurie. Fine, soft rose; beautiful...............

Eugene Verdier. Large, eup-shaped flower, flesh-pink, shaded yellow and salmon; very fresh coloring;

extra fine.............................. 200

Faust. Pretty anemone flower; color tender lilac, center: petals very narrow, flesh shaded with clear salmon; very handsome variety ........................

Felix Crousse. Large, anemone-shaped flower of perfect form; very brilliant red......................

Festiva maxima. Very large, pure white flower, with some blood-red stains in center; tall stalks, beautiful foliage, and very free-flowering. One of the very best white Peonies in cultivation..................

Festiva. Dwarf, pure white, center carmine-spotted... Fimbriata plena. Dark violet, fringed petals; very pretty flower.................................

Floral Treasure. Soft rose; ligules buff, witl tufts of rose petals in center; distinct and fine .............

Formosa. Pretty convex flower; sulphur-white, stigmate lively red; very beautiful variety.............

Formosa alba. Very large full flower; dull white, slightly tinted cream; extra ......................

Fragrans rosea. Sweet-seented, pink variety.......... Fragrantissima. Violet and rose color; very sweet....

$\begin{array}{lll}3 & 50 & \text { Francis Ortegal. Dark purple-crimson; rery large, fine, } \\ 4 & 00\end{array}$ double and sweet ............................ 100 Geldolf. Soft red; extra fine .................... 40

Georges Cuvier. Purple-lilac flowers, silver border .... General Bertrand. Violet-rose center, lined with salmon. $\quad 35$
$60 \quad 6 \quad 00$

$50 \quad 500$


CHOICE NAMED PEONIES, continued

Germain Bigot. This is a new French variety, and one of the most beautiful in our collection. Very large

flower; clear brilliant pink, shaded with salmon ....\$5 00

Globosa. Flesh-pink, chamois center ............. 30

Gloire de Chenoceaux. Large, full flowers in clusters, beautiful satiny rose, lightened with white; rery

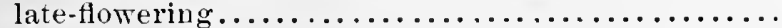

Golden Harvest. Nearest approach to yellow .......... Grandiflora nivea. Very large, pure white flower, shaded with sulphur, lovely rose and salmon, with some stains of carmine; a rariety of perfect loveliness ................................... 125

Grandiflora rosea. Very large, full, convex flower, rose and salmon mixed; beautiful ....................

Grotchen. White and rose, with yellow center.........

Henricartiana. Outside petals violet-rose, those of the center shaded salmon: very beautiful variety.......

Humei rosea. A splendid old sort, with deep rose flowers; one of the latest to bloom....................

Isabel Karlitzsky. Large; delicate rose............

J. B. Rendatler. Large imbricated flower, clear cerisepink, with silver reflex; late variety..............

Jeanne d'Arc. Large flowers of soft rose, sulphur-white and lively rose, center stained carmine, lovely flesh coloring $\ldots \ldots \ldots \ldots \ldots \ldots \ldots \ldots \ldots \ldots \ldots \ldots \ldots \ldots \ldots, 1$

Jeramus. Pink, yellow center................... 50

King of Roses. Deep rose; sweet-scented..............

La Coquette. Large flower of perfect shape; lively beautiful rose, center flesh pink, salmon and carmine; one of the finest........................... 100

La Tulipe. Very large, globular flower, rosy white center, outside of the flower lively carmine, center striped deep carmine; extra fine...................

Lady Bramwell. Silvery rose; very fragrant........ 50

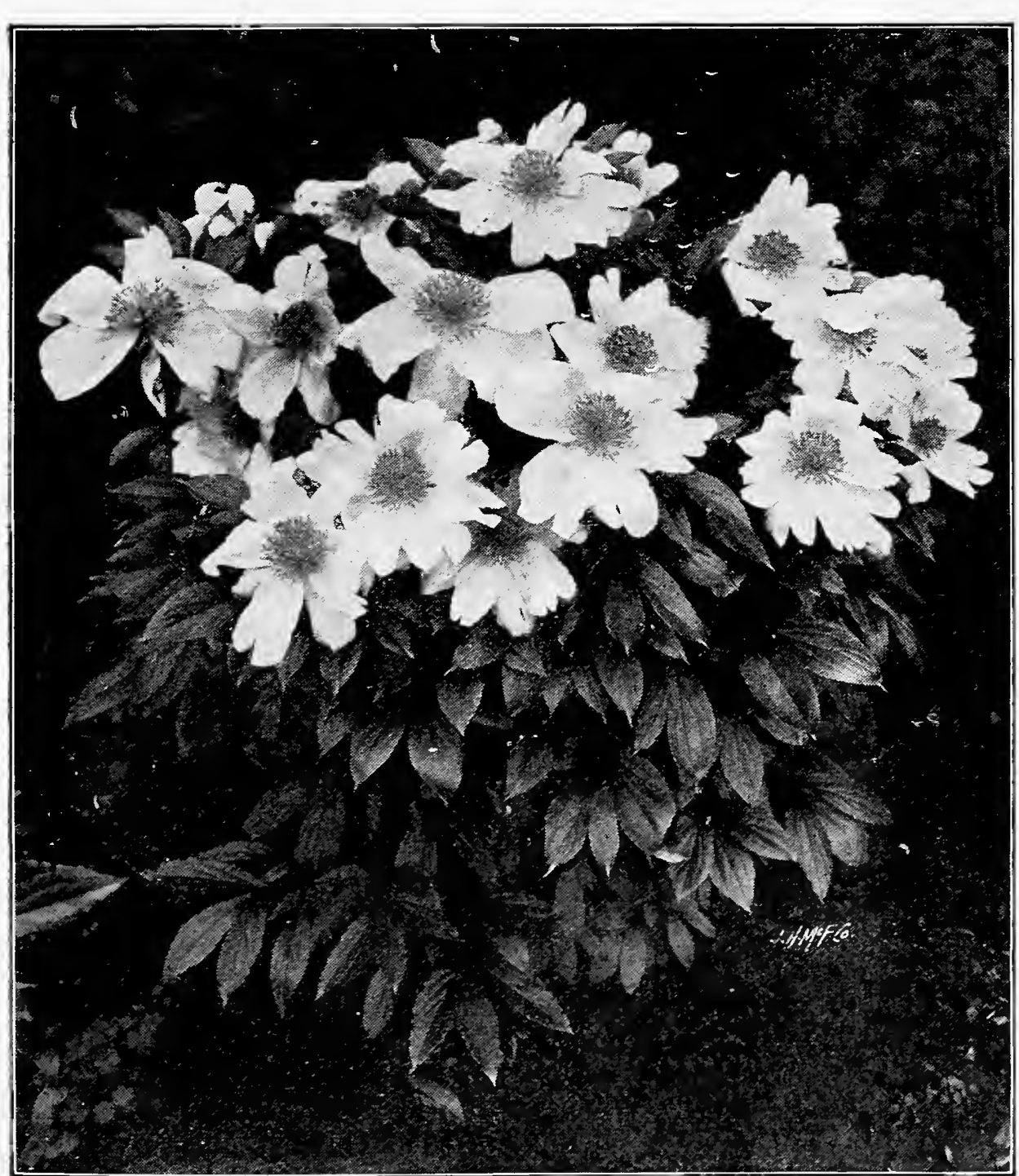

$60 \quad 600$

$75 \quad 800$

L'Innocence. White, rosy onter petals Each Per doz. Louis Van Houtte. Large flower of lively riolet-red; very brilliant; a very beautiful variety ............

M. Chevreul. Large, imbricated flowers, very full; beautiful lilac, shaded pink; rery fresh coloring....

M. Courant. Blush-pink, center lemon-yellow.........

Madam Crousse. Large, perfect flower; pure white;

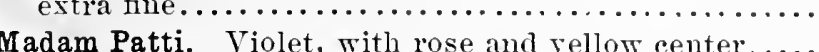

Magnifica. Soft rose, center sulphur edged carmine; dwarf plant; extra fine........................

Maiden Blush. White and light rose; beautiful........

Marechal Vaillant. Very large flower; violet-red; lively

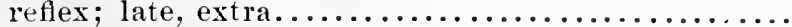

Marie Lemoine. Delicate flesh, changing to white....

Mathilde Mechin. Very pretty anemone flower, compact, ball-shaped, perfect form, carnation-pink mixed with small salmon petals ....................... 100

Maxima rosea plena. Dark carmine-rose; very full.... 75

Meissonier. Very large, double flower with large petals of brilliant purple-amaranth; very beautiful........

Mlle. Renee Dessert. Very large flowers in clusters; beautiful lilac with silvery violet reflex; extra ..... 100

Mme. Aug. Petereau. Large, full flower, beautiful lively satiny pink, silvery reflex on the edge; late variety.

Marie Lemoine (Lemoine). Extra large, free-flowering, ivory white............................. 1 00

Mme. Bucquet. Black relvety amaranth, rery deep color; extra fine ............................ 100

Mme. Calot. Large, peony-shaped flower; very double, carnation-white, tinted yellow ................. 100

Mme. Chaumy. Large flowers in clusters; rose-shaded, large silvery border; very late variety .............

Mme. de Verneville. Very pretty anemone flower, very full; collar of large petals, those of the center very close; carnation-white and sulphur, sometimes carmine; extra.... 100

Mme. de Vatry. Very large flower of perfect shape; color clear carnation, sulphur-white center with carmine stripes; extra fine variety ................. 150

Mme. Ducel. Very large, globular flower, extra full and perfect shape; silvery salmon-pink ....................

Mme. Emile Galle. Large, cup-shaped, imbricated flower, soft lilac, center yellowish white, fresh coloring; extra fine ........................... 150

Model of Perfection. Beautiful soft pink. 50 Modeste Guerin. Broad flower; outside rose, center pink................. 125

M. Dupont. Large, cup-shaped flower, perfect shape, sulphur-white, carmine edge; extra...................... 100

Mme. de Gavin. Large flowers in clusters; very soft flesh pink, touched with lively carmine; extra..................

Mme. Leban. Very large flowers in clus ters, very double; very brilliant cerise petals mixed with salmon ligules, silvery reflex; late variety; extra ......

Ne Pis Ultra. Very large flower of good shape, fresh and lively pink..........

New Giant. An extremely large and showy

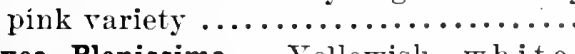

Nivea Plenissima. Yellowish white stainerl carmine; dwarf plant ........ 100

Nobilissima. Fine bright dark rose..... 35

Norfolk (Richardson). Beautiful soft pink; fine-shaped flower ............ 150

0fficinalis. Single; white............ 40

Officinalis alba (Old Double Thite). Blush-white; early-flowering.........

Officinalis rosea (Old Double Rose). Rich bright shining rose; very early..

old Double Crimson. This fine old Peony is very effective when planted in masses; one of the earliest to bloom... per $100, \$ 20$ 
CHOICE NAMED PEONIES, continued

Palassi. Light rose, blush center. 40 ets. each, $\$ 4$ per doz.

Perfection. Outside petals roselilac, inside purple and salmon; sweet. 50 cts. each, $\$ 5$ per doz.

Philomele. Soft satiny rose-color, center composed of saffron-yellow ligules tufted lively red; an elegant flower of remarbahle freshness. $\$ 1$ ea., $\$ 10$ per doz.

Phrynee. White, center light yellow, blotclied red; extra. 75 cts. each, $\$ 8$ per doz.

Plenissima rosea superba. Very full convex flower, beautiful pink and salmon. $35 \mathrm{cts}$. each, $\$ 3.50$ per doz.

Pottsi. Dark purple: sometimes semi-double. $30 \mathrm{cts}$. each, \$3 per doz.

Preciosa Nova. Blush-pink, center shading to white. $25 \mathrm{cts}$. each, $\$ 2.50$ per doz.

Prince de Salm Dyck. Lovely lilac, chamuis center, tufter lilac. 50 cts. each, $\$ 5$ per doz.

Prince Imperial. Very large flowers ; shining purple-scarlet. Very beautiful. 50 cts. each, $\$ 5$ per doz.

Princess Galitzen. Soft carnation, very narrow center, petals of sulphur-yellow; fine. $\$ 1$ each.

Pulcherrima. Large, imbricater flower; riolet-rose, center pink and salmon. Very pretty. 50 cts. each, $\$ 5$ per doz.

Purpurea superba. Large flower, violet and red; very Each Per doz. beautiful ................................. \$0 40 \$4 00

Princess Clothildo. Large cup-shaped flower, very clear carnation; very beautiful......................

R. H. Boggs. Crimson outer petals, with flesh-white center; distinct and fine......................

Reine Victoria. Carnation-white, center clear yellow,

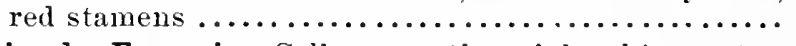

Reine des Francais. Collar carnation-pink, white center,

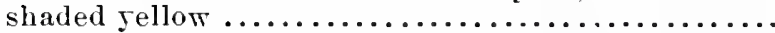

Rosalinde. White, with light rose; extra beantiful.... 100

Rose d'Amour. Large flower of very soft carnation-pink; very fresh color; fine........................ 125

Rosea plena. Double pink...................... 25

Rubra superba (Richardson). Grand globe-shaped flower; purplish crimson; very late. One of the finest reds in cultivation..................... 100

Snowball. Pure white ........................ 50

Solfaterre, Collar of large, pure white petals, those of the center narrow and sulphur-yellow. One of the best .................................. 100

Souvenir de l'Exposition du Mars. Large flower; beautiful lively violet-red, with brilliant silrery reflex; extra fine.............................. I 00

Souvenir de l'Exposition Oniverselle. Very large, imbricated flower of perfect form; rich cerise; rery

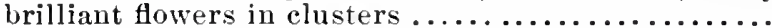

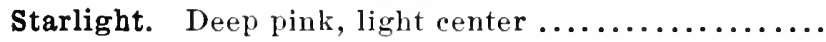

Sweetheart. Red and white, with rose center ..........

Sarah Bernhardt. Large flowers of fine effect; corolla cf large petals, lively violet-rose, center salmon ....

Tenuifolia. Same as following rariety, but with beauti-

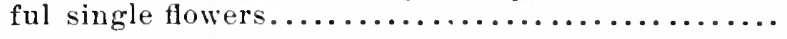

Tenuifolia flore pleno. Deeply cut, fringe-like foliage; flowers bright scarlet-crimson; rare and fine........

Thisbe. Flesh-pink

Triomphe de l'Exposition de Lille. Large, imbricated flower; soft carnation-pink, with white reflex, carmine center; very fresh coloring. One of the very

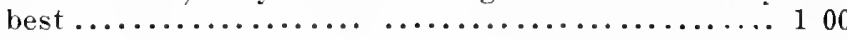

Triomphe du Nord. Violet-rose, lilac sharle ........ 30
50500

50500

$50 \quad 500$

75

1000

$50 \quad 500$

(3)

1000 300

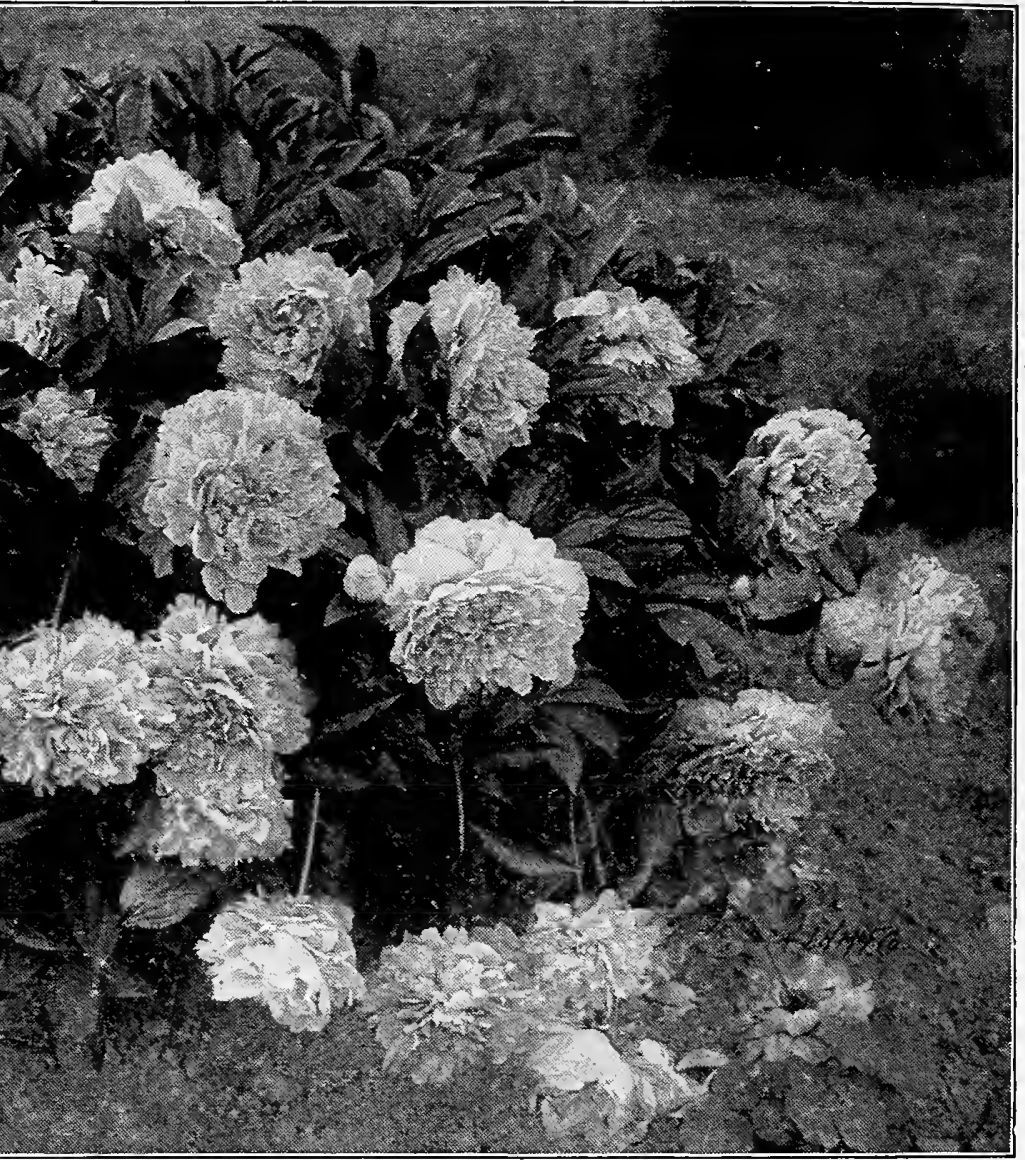

SPECIMEN PLANT OF DOUBLE PEONY.

Triumphans, Pink, shaded lilac, chach Perdoz. Van Dyck. Large flower; color very fresh salmonpink, center tinterl with salmon aud chamois; extra

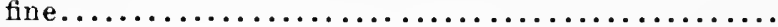

Victoire Modeste. Large flowers of violet-rose, center petals marked with laıge salmou lines.............

Victoire de L'Alma. Large flower of perfect form, purplish violet-scarlet, silvery reflex; extra ............

Virginie. Large flower, pink tinted lilac, very fresh

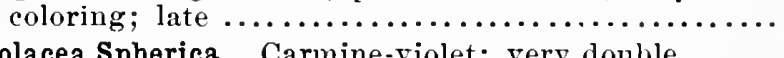

Washington, Clear red, center soft salmon-pink; beau-

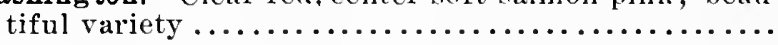

Whitleyi. White; large and sweet $\ldots \ldots \ldots \ldots \ldots \ldots \ldots$ $75 \quad 800$ 75

50500

$75 \quad 750$

75800

$50 \quad 500$

$35 \quad 350$

Wilhelmina. Fine soft rose; extra large ......... $75 \quad 750$

\section{KELWAY PEONIES}

Kelway \& Son are the most famous growers of Peonies in the world, and the following varieties are what they consider the very best of their new varieties. These Peonies are imported to order only, and orders must be received not later than October 1 for fall delivery or May 1 for spring delivery. Prices include duty and all charges,

Agnes Mary Kelway. Light rose guard petals, yellow petaEach loids, with a rose tutt: extra fine. First.class Certificate.

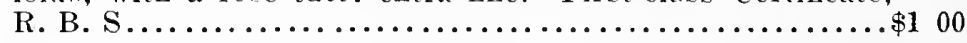
Alonzo. Deep crimson. First-class Certificate, R. B. S...... 200 Bunch of Perfume. A full double flower, of a vivid rich rose color; exceedingly sweet-scenterl. First-class Certificate,

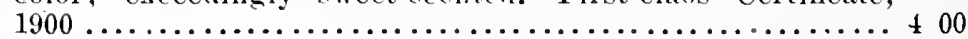

Baroness Schroeder. Lovely flesh-pink. First-class Certificate.

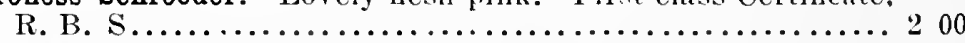
Cyclops. Purple-crimson. First-class Certificate, R. B. S..... 100 Duchess of Teck. An excellent variety; large and of good form, attractively colored, creany white and bright pink. Firstclass Certificate, R. B. S. Award of Merit, R. H. S...... 200 Duke of Cambridge. A very handsome bright crimson flower; a superb variety; the very best of its color ............2 00 Duke of Clarence. Cream, slightly flushed pink. First-class

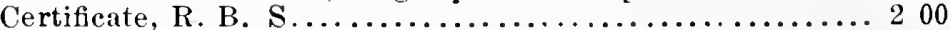




\section{KELWAY PEONIES, continued}

Duke of Devonshire. "A large rariety of deep rose color, with large outer guard petals and dense center."-Journal of Horticulture. "Duke of Deronshire is a fine large double variety, with deep rose guard petals and a mass of small inner petals of the same color."-Gardeners" Magazine. Award of Merit, R. H. S......................\$3 00

Ella Christine Kelway. Beautiful soft lavenderflesh color, enclosed in a large guard petal; very large and full and of perfect form, very sweetly perfumed "Ella Christine Kelway, a very handsome herbaceous kind, with white blossoms faintly tinted with salmon- pink. The variety is remarkable for its fullness and the great solidity of the blooms."-The Garden. Award of Nierit, R. H. S., June 14,

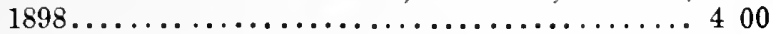

Glory of Somerset. Soft pink, large, beautiful. First-class Certificate, R. H. S............ 300

Harpasus. Pure white................... 100

Joan Seaton. Double, bright cherrr-rose, each petal edged with a lighter color; rose-shaped flowers, showing anthers amongst the petals: nicely scented. Certificate of Merit, R. B. S., $1897 \ldots \ldots \ldots \ldots \ldots \ldots \ldots \ldots \ldots \ldots \ldots \ldots \ldots \ldots \ldots$. and lorely sort, and very sweet. First-class Certificate, R. B. S.................... 200

Lady Beresford. The petals are tipped with carmine; rery sweet. "A large-Howered variety of a soft blush-pink shade, delicate and beautiful; a very fine-habited strong plaut."-The Garden. "Lady Beresford is a huge white flower, with a tinge of buff at the base of the broad soft segments: a magnificent bloom."Gardentr"s ilfagazine. Award of Merit, R. H. S .......................... 300

Lady Carrington. Flesh very fine, sweet-smelling. First-class Certificate, R. H. S..........2 200

Lady Gwendolin Cecil. A very delicate larenderflesh; a beautiful flower: late-flowering. Firstclass Certificate, R. B. S. ................. 300

Langport Queen. Of a beautiful rosy blush color, suffused with pink; very fine............... 200

Leonard Kelway. Pink guard petals, enclosing a conrex cushion of cream-colored petals finished off by a pink crest. First-class Certificate,

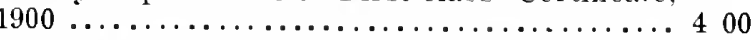

Limosel. Very bright, clear, light lilac-rose; very large flower: full double, with a broad guard petal and narrower petals in the center, sweetscented. Certificate of Merit, R. B.S. $1897 \ldots 300$

Lottie Collins. Deep purple : early-flowering. A ward of Merit, R. H. S. .................2 200

Lyde. Rose-color center, tinted pink; distinct and extra fine. First-class Certificate, R.H.S. 200

Miss Brice. Rose guard petals, yellow and rose petaloids, rose Each tuft. First-class Certificate, R. H. S................\$2 00

Miss Salway. White guard petals, sulphur center; rery fine. First-class Certificate, R. H. S., and Certificate of Merit, R. B. S.................................. 200

Moonbeam. Large white, tufted in the center. Award of Merit,

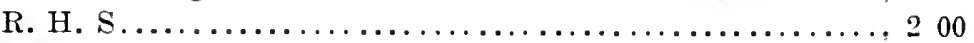

Mr. Manning. Deep glowing crimson. Certificate of Merit, R. B. S................................... 200

Paderewski. Bright pink; very sweet-smelling. First-class Certificate, R. B. S............................. 200

Prince George. Fine purple. First-class Certificate, R. B. S. 200

Sir T. J. Lipton. Large flower, with golden stamens appearing in the center of bright rosy crimson petals. "A buge double, bright rosy carmine flower of great merit."-Gardening World. Certificate of Merit, R. B. S., 1897............ 300

Suminer Day. Cream white. Certificate of Merit, R. B. S... 300

Venus. Flesh-pink, with shades of salmon; most delicate; a beautitul colored plate of this rarietr has appeared in The

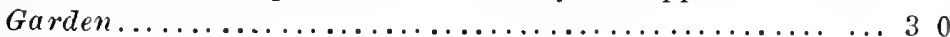

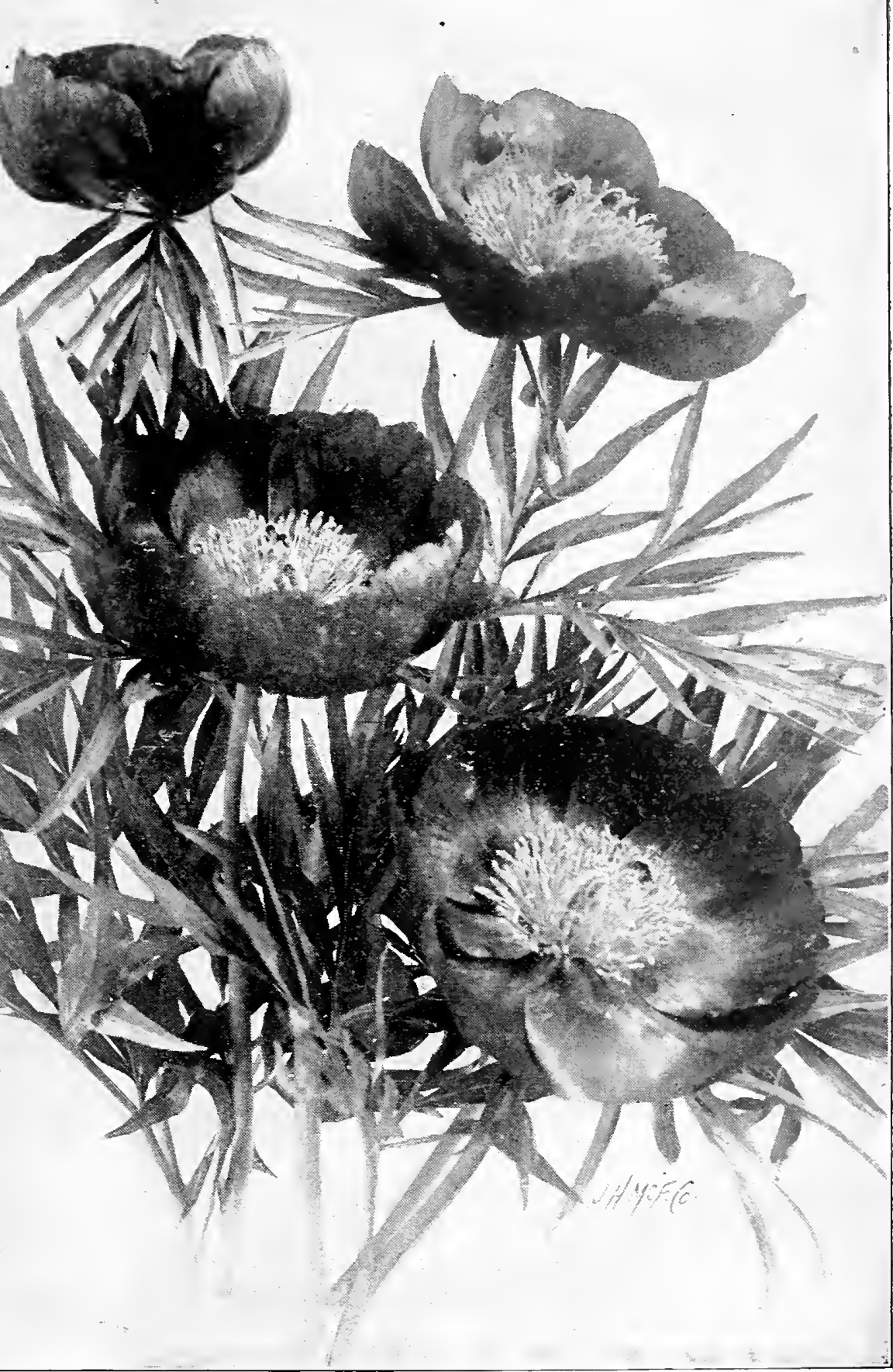

PEONY TENUIFOLta (See page 3?)

\section{SINGLE VARIETIES}

Autumnus. Purple. First-class Certificate, R. H. S., Manches- Each ter. First-class Certificate. R. B. S................. $\$ 130$

Bridesmaid. Single; white........................ 300

Dorothy. Delicate pink: very charming............... 200

Fairy. Rosy white ............................... 200

Felix Holt. Pink................................ 200

Lady Wimborne. Fine pearls blush-pink................. 2 00

Pride. Rich crimson; a most striking flower............... 200

Stanley. A gorgeous rariety of the riclest and deepest maroon crimson, with pure gold-color stamens. First-class Certificate, R. B. S, and Award of Merit, R. H. S............. 300

The Czar. Deep purple-crimson.................... 130

Tinted Venus. Bright pink. First-class Certilicate, R. B. S. . 200

Viscount Cross. Deep cherre-red. "A charming single rariety, of a bright cherry hue."-Gardening World. First-class

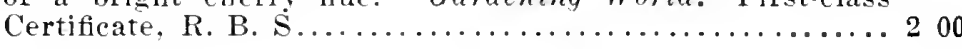

\section{MIXED PEONIES}

This mixture is made up from rarieties of which we have not sufficient to catalogue. It does not contain the best rarieties, but the quality is extremely good for the low price quoted. $20 \mathrm{cts}$. each, $\$ 2$ per doz., $\$ 1$ per 100 . 


\section{Special Offer of Ornamental Flowering Shrubs}

\section{SHIPPED FROM STATION NEAR PITTSBURG}

I want to encourace the planting of shrubs. To be effective they slould be planted in masses, but when dealers or nurserymen ask fifty cents each for easily grown shrubs there is not much encouragenent to plant them freely. I have made arrangements by which I can supply my customers with well-grown shrubs in the best varieties at the extremely low prices quoted below, but in every instance the selection of varieties must be left to me; however, in ordering, if it is stated that certain shrubs are not wanted they will not be sent. I guarantee these shrubs to be satisfactory in both quality and variety.

SPECIAL OFFER A. -50 shrubs in fine assortment of 15 varieties, $\$ 7$.

SPECIAL OFFER B. -100 shrubs in fine assortment of 20 varieties, $\$ 12$.

MY GENERAL CATALOGUE, containing full price list of Trees and Shrubs, will be sent free on request

\section{Low Prices for Hydrangeas}

\section{SHIPPED FROM STATION NEAR PITTSBURG}

Prices good until stock is exhausted. Fall or spring delivery. No more popular plant or shrub has ever been sent out than Hydrangea paniculata grandiflora, and 1 take great pleasure in offering my customers a large stock of splendid vigorous plants at such extremely low prices that it will enable then to plant this effective shrub in quantity.

People who have only seen this Hydrangea grown singly as specimens have no conception of how beautiful and effective it is when planted in masses. They are planted in this way at Newport, R. I., which is famous for its fine gardens, and almost as famous for its Hydrangeas. These Hydrangeas can be planted in connection with other shrubbery or in isolated beds, in the same manner as cannas, caladiums or other strong-growing bedding plants. 'They are perfectly hardy, and once planted they are a permanent addition to the lawn or garden. When grown in beds or groups they should be planted about two feet apart in rery rich soil, which should be liberally enriched annually with rotten stable manure, and in the early spring, before they commence to grow, cut back so as to leave only two or three inches of the new growth of the previous season; if extremely large flowers are desired, cut out some of the weakest shoots after growth has commenced. Treated in this manner they will pro duce enormous panicles of flowers, and the beds will be a solid mass of bloom. They bloom profusely the same season planted. Try them. You will find them more than satisfactory.

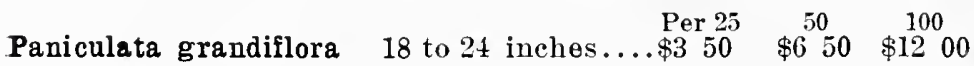
Paniculata grandiflora. 2 to 3 feet, extra
large and bushy...................... $500 \quad 860 \quad 1500$

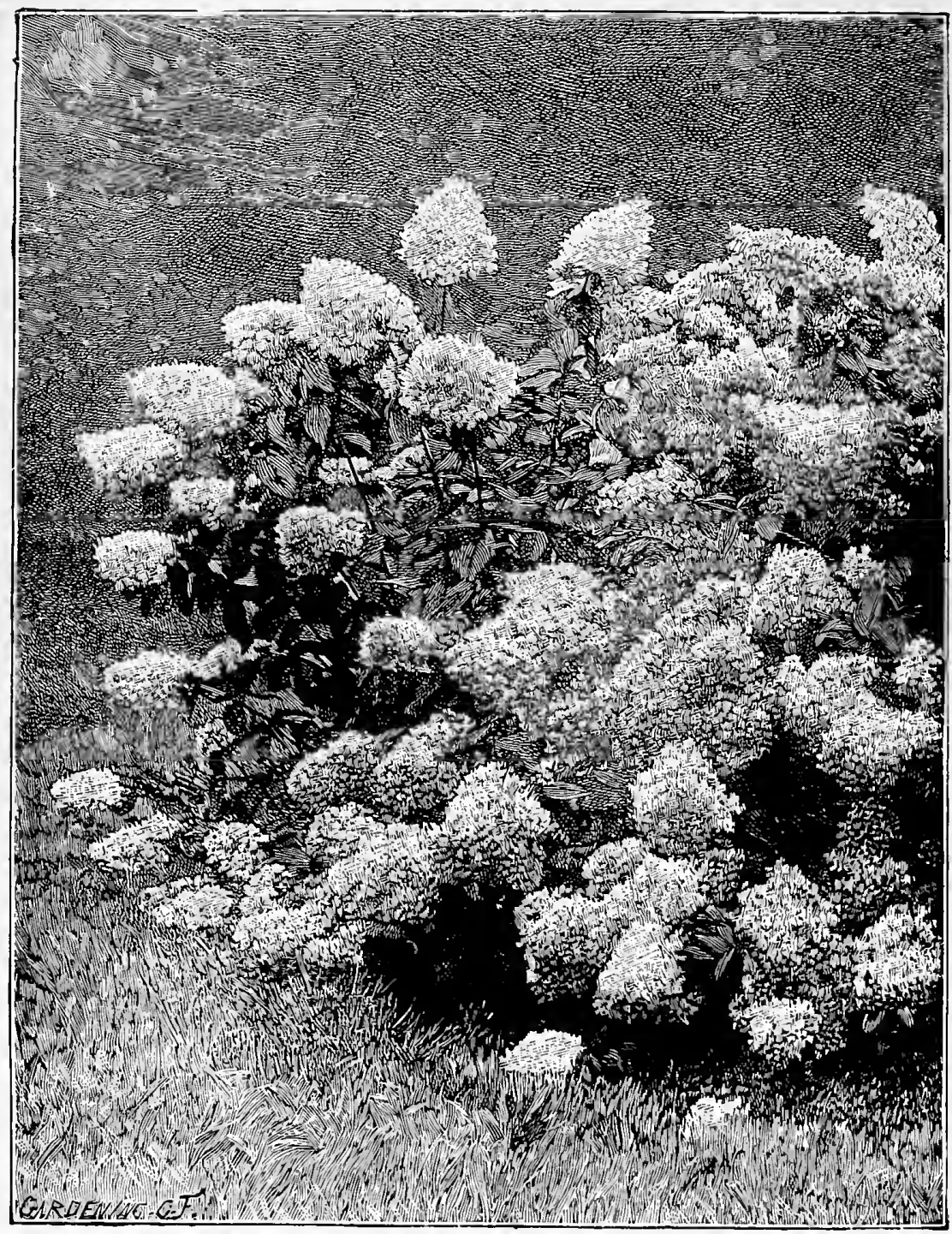

GROUP OF HARDY HYDRANGEAS ON LAWN From "The English Flower Garden"

All of the above will bloom the first year, and are superior to the stock commonly retailed at 25 ets. to $\$ 1$ each.

It is suggested that when smaller quantities are wanted two or three club together in ordering.

For prices on smaller quantities, see list of Hardy Shrubs.

\section{Hollyhocks}

Have you ever noticed that painters ehoose single Hollyhors as subjects for floral painting oftener than any other flower? I suppose the reason for this is that artists have a keener appreciation of beanty than other people and recognize that the common, and $I$ fear sometimes despised, Single Hollyhock, is one of the most stately, pieturesque and beautiful plants in the world. Nothing can be more effective than a large group or mass of single Hollyhocks, and once planted tliey will literally take care of themselves, eren if planted in the grass. I know of patches that have not been cultivated or disturbed in any way for twenty years. Notwithstanding their great beauty and usefulness, Single Hollylioeks are not offered for sale by any nurseryman in America, and the seed is not to be had from any seedsman. I am determined to have Single Hollyhocks for my landscape gardening work, so I collected seed from old-fashioned (they will soon be newfashioned) gardens and had thousands of plants grown for my eustomers. Some people think Double Hollyhocks are more beautiful than Single. They are mistaken; although the doubles are beautiful, but inelined to be top-heary, often need staking and are liable to disease. Hollyhocks planted in the fall will bloom well the following summer.

Per doz. 100 Best Large-flowered, Single. All colors mixed....... $\$ 125 \quad \begin{array}{lll} & 25 & 00\end{array}$ Best Double Grown, Chater's Famous Strain. In separate colors: white, sulphur-vellow, scarlet, rose,

Isabel color, salmon-rose, crimson, golden rellow,

dark erimson, eherry-red and peach................ 150

New Everblooming......................... 150

1000

1000
New Allegheny. Inmense semi-double flowers, with fringed edges; very beautiful...................each, 20 ets.... $\$ 200$ Ficifolia (The Fig-leared Hollyhock). With bright vellow flowers ....................................... 250

Primrose Queen. A splendia addition; harge, bright single primrose-yellow flowers ............................ 250 


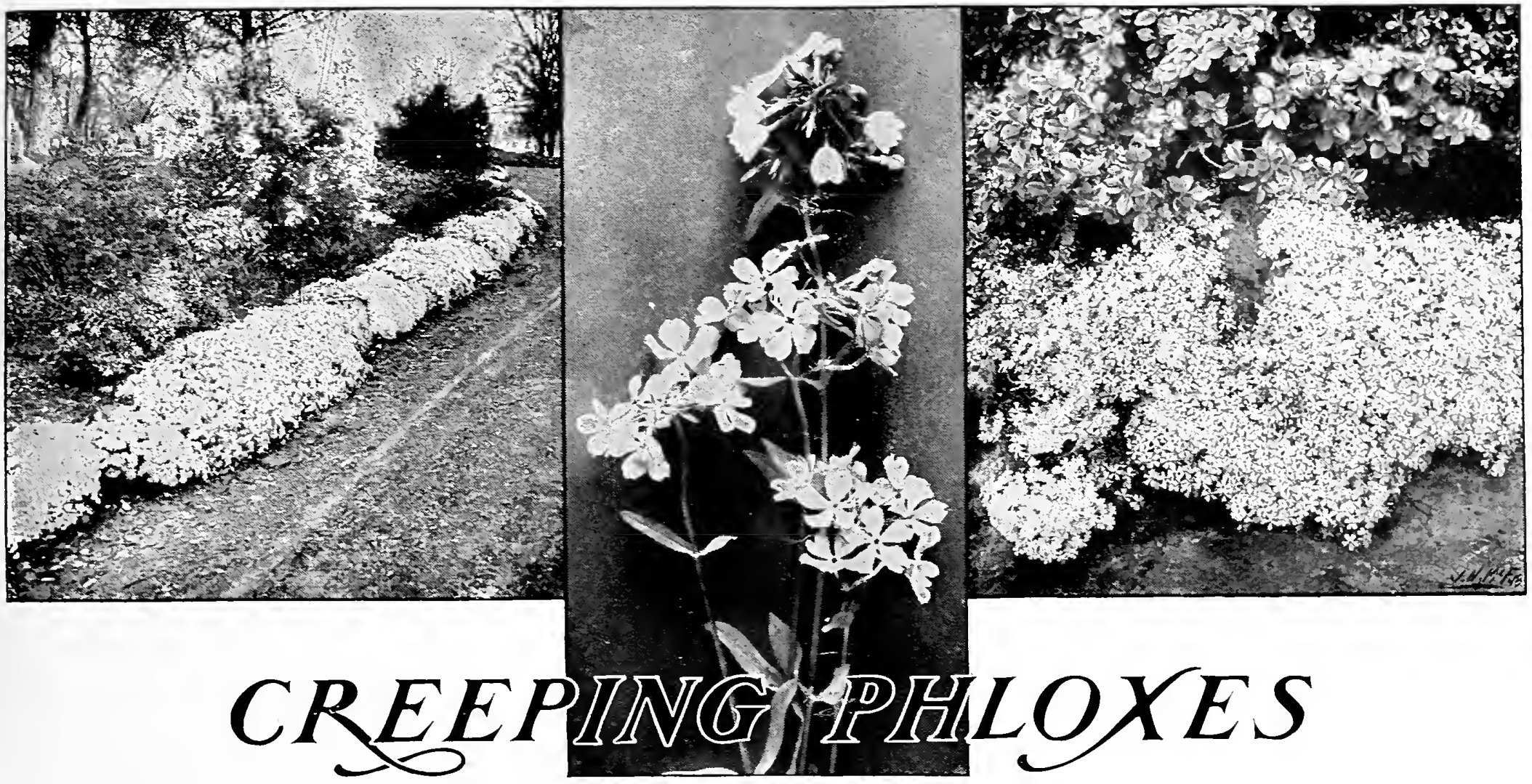

The Creeping Phloxes have a charming beauty all their own, and, for general usefuluess, are not to be surpassed by any flower that grows. They will thrive in any situation, except a very shady one; and in any soil, no matter how poor, unless it is very wet. They are absolutely hardy without protection; hardy last winter, which killed millions of plants and shrubs, and even trees. They have neat evergreen foliage, and when in bloom, which is during the month of May, their multitude of lovely pink, white or blue flowers cover everything beneath as completely as a carpet covers a floor. They bloom again in September, but not so freely. For covering rocky hillsides, sandy slopes, bare ground too poor to grow anything else, or for edging borders of hardy plants or shrubs, they are unsurpassed. Our President literally uses these plants by hundreds of thousands in his landscape work, and wherever he uses them there is a large demand for them. Just think of it! The splendid, inexpensive hardy plants, which increase fourfold every year, are almost unknown, while commonplace geraniums, with not one-tenth their beauty, are planted by the million, and must be planted annually.

The Creeping Phloxes can be planted advantageously in September and until the fifteenth of October. As we grow them by the acre, we can offer them in quantity at very low prices, which are given below.

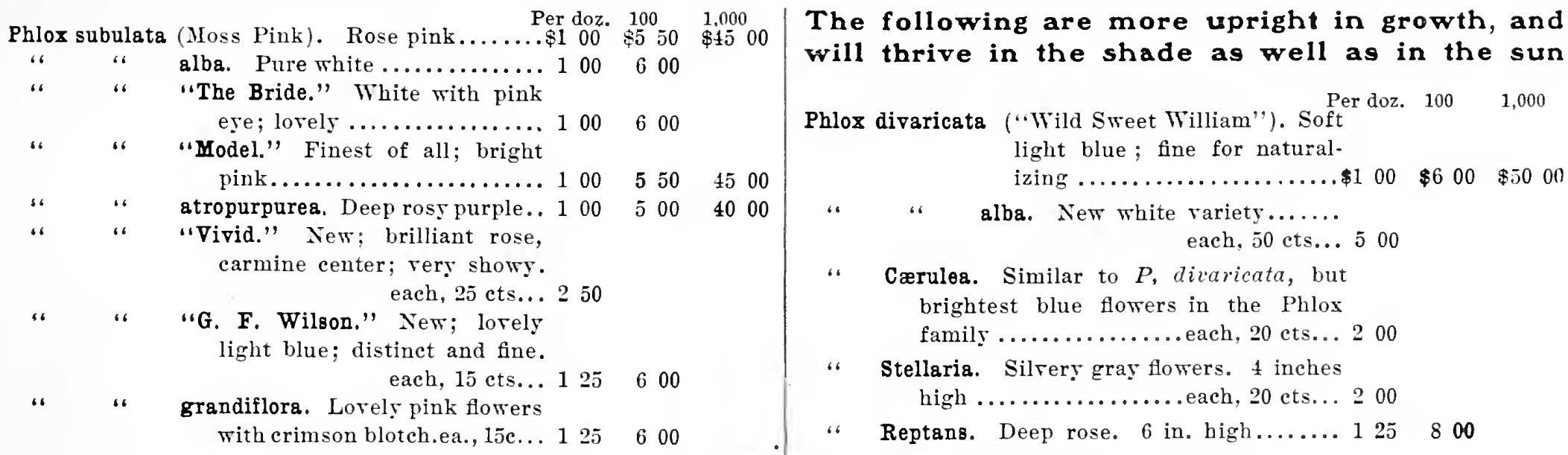

\section{Special Offers of Hardy Plants in Variety}

The following very low prices are for the purpose of equalizing stocks of Hardy Plants. They will be of the best and most desirable varieties and the plants of the best quality, but in evers instance the selection of rarieties is to be made by us. Howerer, as before stated, if purchasers will name the things they have or don't wish, these will not be included in the selection.

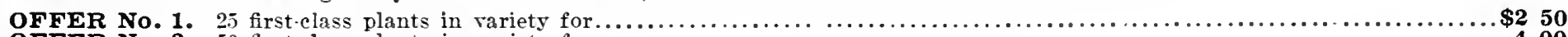

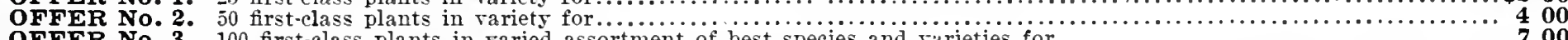

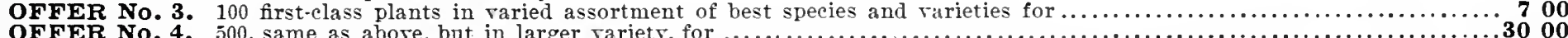

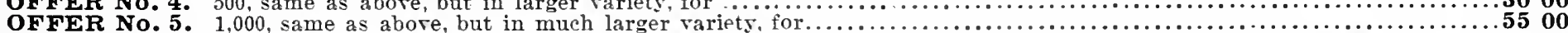

Our General Catalogue, contalning complete llst of Hardy Plants, with descriptions and prices, will be sent free on request 


\title{
A PLEA
}

\section{By J. WILKINSON ELLIOTT}

TTENDER plants predominate in so many modern gardens that lovers of flowers should be grateful to $\mathrm{Mr}$. J. Wilkinson Eliiott for bringing forth an argument, in the shape of a sensible and practical book, in favor of our native asters, lilies, hollyhocks and other hardy plants. This book is sure to appeal to all garden makers.

\section{PRESS COMMENTS}

\begin{abstract}
"Certainly one of the most sensible as well as beautifully illus. trated nature books that the season has to offer."-Chicago RecordHerald.

"An irresistible combination of sensible suggestions and splendid illustrations."-Los Angeles Herald.

"The price is incredibly small considering the mechanical perfection of the book." - Nashville A merican.

"Such a book is an inspiration to the inert land-owner, and has real help to offer the energetic one."-Farm, Field and Fireside.

"Should be in the hands of every owner of a country place."-The Argonaut.
\end{abstract}

PRACTICAL AND WELl ILLUSTRATEd. PRICE, NET, \$1.60

DOUBLEDAY, PAGE \& COMPANY, Publishers, 34 Union Square, New York

\section{SPECIAL OFFER}

TE should like very much to have every one of our customers receive a copy of the book advertised above, and with this end in view we have made arrangements with the publishers by which we can supply our customers with it at a nominal price. With orders for trees, shrubs, plants or bulbs amounting to two dollars or more, a copy of the book bound in boards may be added for seventy-five cents, but only one copy can be ordered at this price, or every one who sends to us a subscription to that splendidly illustrated magazine, "Country Life in America," will receive a copy of the book, "A Plea for Hardy Plants," free of cost; or, in other words, the magazine and the book will be sent for the price of the magazine alone, which is four dollars per year.

\section{ELLIOTT NURSERY COMPANY}

\section{8}

\section{LANDSCAPE GARDENING}

\begin{abstract}
ThE majority of American suburban grounds are laid out and planted without the aid of professional advice. The result is almost always unsatisfactory, although often the expenditure would have secured most beautiful results if directed by skilled advice. I do landscape gardening-do it for people of exacting taste, to whom I refer. I make the plans, with estimates, purchase the stock necessary and superintend the work. I do any one or all these things, satisfactorily as to results, moderately as to cost. For small grounds I can make satisfactory plans if furnished with a plat drawn to scale. For large grounds, and where extensive improvements are desired, a personal visit would be necessary and can be arranged for on reasonable terms. I can make no plans during the month of April.
\end{abstract}

\section{J. WILKINSON ELLIOTT}

\section{LANDSCAPE ARCHITECT}

